\title{
THE SOLAR NEIGHBORHOOD. XXXIII. PARALLAX RESULTS FROM THE CTIOPI 0.9 m PROGRAM: TRIGONOMETRIC PARALLAXES OF NEARBY LOW-MASS ACTIVE AND YOUNG SYSTEMS
}

\author{
Adric R. Riedel ${ }^{1,2,11,12}$, Charlie T. Finch ${ }^{3,11}$, Todd J. Henry ${ }^{4,11}$, John P. Subasavage ${ }^{5,11}$, Wei-Chun JaO ${ }^{4,11}$, Lison Malo ${ }^{6}$, \\ David R. Rodriguez ${ }^{7}$, Russel J. White ${ }^{4}$, Douglas R. Gies ${ }^{4}$, Sergio B. Dieterich ${ }^{4,11}$, Jennifer G. Winters ${ }^{4,11}$, \\ Cassy L. Davison ${ }^{4}, 11$, Edmund P. Nelan ${ }^{8}$, Sarah C. Blunt $^{2,9}$, Kelle L. Cruz $^{1,2}$, Emily L. Rice ${ }^{2,9}$, and Philip A. Ianna ${ }^{10,11}$ \\ ${ }^{1}$ Department of Physics and Astronomy, Hunter College, The City University of New York, 695 Park Avenue, New York, NY 10065, USA \\ ${ }^{2}$ Department of Astrophysics, American Museum of Natural History, Central Park West at 79th Street, New York, NY 10024, USA \\ ${ }^{3}$ Astrometry Department, U.S. Naval Observatory, Washington, DC 20392, USA \\ ${ }^{4}$ Department of Physics and Astronomy, Georgia State University, P.O. Box 5060, Atlanta, GA 30302-5060, USA \\ ${ }^{5}$ United States Naval Observatory, Flagstaff, AZ 86001, USA \\ ${ }^{6}$ Département de Physique et Observatoire du Mont-Megantic, Université de Montréal, C.P. 6128, Succursale Centre-Ville, Montréal, QC H3C 3J7, Canada \\ ${ }^{7}$ Departamento de Astronomia, Universidad de Chile, Casilla 36-D, Las Condes, Santiago, Chile \\ ${ }^{8}$ Space Telescope Science Institute, Baltimore, MD 21218, USA \\ ${ }^{9}$ Department of Engineering Science and Physics, College of Staten Island, 2800 Victory Boulevard, New York, NY 10314, USA \\ ${ }^{10}$ Department of Astronomy, University of Virginia, Charlottesville, VA 22904, USA \\ Received 2013 August 27; accepted 2013 December 30; published 2014 March 14
}

\begin{abstract}
We present basic observational data and association membership analysis for 45 young and active low-mass stellar systems from the ongoing Research Consortium On Nearby Stars photometry and astrometry program at the Cerro Tololo Inter-American Observatory. Most of these systems have saturated X-ray emission $\left(\log \left(L_{X} / L_{\mathrm{bol}}\right)>-3.5\right)$ based on X-ray fluxes from the ROSAT All-Sky Survey, and many are significantly more luminous than main-sequence stars of comparable color. We present parallaxes and proper motions, Johnson-Kron-Cousins VRI photometry, and multiplicity observations from the CTIOPI program on the CTIO $0.9 \mathrm{~m}$ telescope. To this we add low-resolution optical spectroscopy and line measurements from the CTIO $1.5 \mathrm{~m}$ telescope, and interferometric binary measurements from the Hubble Space Telescope Fine Guidance Sensors. We also incorporate data from published sources: $J H K_{S}$ photometry from the Two Micron All Sky Survey point source catalog, X-ray data from the ROSAT All-Sky Survey, and radial velocities from literature sources. Within the sample of 45 systems, we identify 21 candidate low-mass pre-main-sequence members of nearby associations, including members of $\beta$ Pictoris, TW Hydrae, Argus, AB Doradus, two ambiguous $\approx 30$ Myr old systems, and one object that may be a member of the Ursa Major moving group. Of the 21 candidate young systems, 14 are newly identified as a result of this work, and six of those are within $25 \mathrm{pc}$ of the Sun.
\end{abstract}

Key words: open clusters and associations: general - parallaxes - solar neighborhood - stars: low-mass - stars: pre-main sequence

Online-only material: color figures

\section{INTRODUCTION}

Over the past $20 \mathrm{yr}$, a variety of loose associations have been discovered, with distances $(<100 \mathrm{pc})$ much closer than any star-forming region, and ages $(\sim 100 \mathrm{Myr})$ much younger than any comparably close moving group (e.g., Ursa Major; King et al. 2003). These associations include such well-studied groups as TW Hydra (de la Reza et al. 1989; Gregorio-Hetem et al. 1992), $\beta$ Pictoris (Barrado y Navascués et al. 1999), TucanaHorologium (Zuckerman et al. 2001), Argus (Torres et al. 2003), AB Doradus (Zuckerman et al. 2004), Carina (Torres et al. 2008), and Columba (Torres et al. 2008).

Most of the currently known members of these associations are solar-type or hotter stars, reflecting a bias toward bright stars that are surveyed in the proper motion catalogs, Hipparcos and Tycho-2. We are likely left without information on most of the members of these associations; for instance, $\mathrm{M}$ dwarfs make up at least $75 \%$ of all nearby stars (Henry et al. 2006), but make up less than half of the known members of young associations (see Table 1).

\footnotetext{
${ }^{11}$ Visiting Astronomer, Cerro Tololo Inter-American Observatory. CTIO is operated by AURA, Inc. under contract to the National Science Foundation. 12 ar494@hunter.cuny.edu
}

The dearth of $\mathrm{M}$ dwarfs is a distinct issue with star formation theory, and presents difficulties with our understanding of young associations. M dwarfs, because of their lower masses, should be more easily scattered by dynamical interactions than solar-type stars, and thus the current spatial and kinematic boundaries of the associations will not necessarily contain many of the associated stars. By virtue of numbers, they will better inform the initial mass function (IMF) measurements of young associations, which currently appear to be very different from the field IMF (Schlieder 2011). M dwarfs provide an advantage for exoplanet research because they are redder and dimmer, which enhances the contrast between the stars and any forming planets in their star systems. Finally, M dwarfs take significantly longer to reach the main sequence (at least $200 \mathrm{Myr}$; Dotter et al. 2008), which makes it easier to identify and obtain precise ages for young M dwarfs.

To address the issue of missing $\mathrm{M}$ dwarfs, we present the results of a new survey of young and active $\mathrm{M}$ dwarfs, as part of the Research Consortium On Nearby Stars ${ }^{13}$ (RECONS) exploration of the solar neighborhood. We present 45 nearby star systems with M dwarf primaries (35 with new astrometry

\footnotetext{
13 http://www.recons.org
} 
Table 1

Young Star Statistics

\begin{tabular}{|c|c|c|c|c|c|c|}
\hline \multirow[t]{2}{*}{ Association } & \multicolumn{3}{|c|}{$\mathrm{All}^{\mathrm{a}}$} & \multicolumn{3}{|c|}{ Within $25 \mathrm{pc}^{\mathrm{b}}$} \\
\hline & Known Members & M+ Dwarfs & New Members & Known Members & M+ Dwarfs & New Members \\
\hline$\epsilon$ Chameleontis & 40 & 19 & 0 & 0 & 0 & 0 \\
\hline TW Hydra & 41 & 31 & 2 & 0 & 0 & 0 \\
\hline$\beta$ Pictoris & 111 & 68 & 7 & 18 & 16 & 2 \\
\hline Octans & 15 & 0 & 0 & 0 & 0 & 0 \\
\hline Tucana-Horologium & 90 & 26 & 0 & 3 & 2 & 0 \\
\hline Columba & 74 & 20 & 0 & 0 & 0 & 0 \\
\hline Carina & 34 & 13 & 0 & 4 & 4 & 0 \\
\hline Argus & 123 & 53 & 1 & 25 & 21 & 1 \\
\hline AB Doradus & 182 & 74 & 1 & 35 & 24 & 1 \\
\hline Ursa Major & 75 & 8 & 1 & 26 & 8 & 1 \\
\hline Unknown & & & 2 & & & 1 \\
\hline
\end{tabular}

Notes. The known members of nearby young associations, with new discoveries in this paper.

${ }^{a}$ Members are as defined in the following source papers: King et al. (2003), Zuckerman \& Song (2004), Mamajek (2005), Torres et al. (2008), da Silva et al. (2009), Shkolnik et al. (2009, 2011, 2012), Lépine \& Simon (2009), Rice et al. (2010), Schlieder et al. (2010, 2012b, 2012c), Desidera et al. (2011), Kiss et al. (2011), Riedel et al. (2011), Rodriguez et al. (2011), Zuckerman et al. (2011), McCarthy \& White (2012). Quality of membership is as decided in the source paper and has not been re-evaluated here.

b $25 \mathrm{pc}$ is determined by trigonometric parallax if possible; otherwise, from published kinematic, photometric, or spectroscopic distances (in that order).

and photometry) observed during the Cerro Tololo InterAmerican Observatory Parallax Investigation (CTIOPI). Our analysis of youth is based on absolute trigonometric parallaxes, Johnson-Kron-Cousins VRI photometry, spectral types, variability, kinematic analyses, and surface gravity estimates. The systems discussed herein include both known and new candidate pre-main-sequence members of the $\beta$ Pictoris, TW Hydra, Tucana-Horologium, Columba, Argus, AB Doradus, and Castor associations.

Identifying young stars (specifically, pre-main-sequence young stars) is a complicated process. There are many signatures of youth that can be detected in $M$ dwarf stars. Unfortunately, there is no single indicator that completely describes youth, and none of the parameters are foolproof. Lithium equivalent widths (EWs), spectral accretion signatures, and protoplanetary disks are all only found in young stars, but they are short-lived effects, and stars can lack those properties and still be pre-main-sequence objects. Conversely, the other parameters (overluminosity, low surface gravity, chromospheric activity) are long-lived in $\mathrm{M}$ dwarfs and the presence of that signature does not necessarily mean the star is young-particularly, most forms of stellar activity can also be induced by magnetic interactions with a close binary. Our analysis must therefore use multiple independent lines of evidence to identify young stars, similar to recent efforts by Shkolnik et al. $(2009,2012)$.

The key to our present analysis is trigonometric parallaxes: with parallaxes, we have significantly improved constraints on the kinematics of the systems, AND (along with our photometry) we can use the positions of the constituent stars on an H-R diagram with confidence to determine if a system falls along a particular association's isochrones. With our low-resolution spectroscopic data, we can measure spectroscopic features sensitive to surface gravity. We can also measure activity features, though the latter are less useful indicators for M dwarfs.

In Section 2, we discuss the sample selection. In Section 3, we discuss the observations and reductions of the data in this paper. Section 4 describes the methods we used to determine the ages and association memberships of these objects, and in Section 5 we discuss the outcome of the youth analysis carried out on our stars.
This is the 13th paper publishing parallax results from the ongoing CTIOPI program ${ }^{14}$ at the CTIO $0.9 \mathrm{~m}$ telescope.

\section{THE SAMPLE}

The sample of 45 star systems in this paper was drawn from the hundreds of targets in the CTIOPI parallax target list. CTIOPI, by virtue of its location, is limited to objects at declinations south of $+30 \mathrm{deg}$. By using the Tektronix imager at the CTIO $0.9 \mathrm{~m}$ telescope, CTIOPI is further limited to stars between 9th and 18th magnitudes in at least one of our three Johnson-Kron-Cousins VRI filters. CTIOPI generally targets $\mathrm{M}$ dwarf stars whose estimated distances-either from literature, or from our own photometric distance estimates (Hambly et al. 2004; Henry et al. 2004)_place them within 25 pc of the Sun. The targets in this paper are consequently all nearby bright $\mathrm{M}$ dwarfs (see Table 2, column 16).

From the CTIOPI target list, we identified potentially young stars using X-ray saturation $\left(\log \left(L_{X} / L_{\text {bol }}\right)>-3.5\right)$ as an indicator of chromospheric activity, and overluminosity-herein defined as being more than 1 mag brighter than a single mainsequence star of comparable colors - as an indicator of low surface gravity. As shown by Zuckerman \& Song (2004), X-ray emission in $M$ dwarfs is saturated and remains at the $\log \left(L_{X} / L_{\text {bol }}\right) \approx-3.0$ level in stars at least as old as the Hyades (600 Myr), the oldest of the associations we consider here. Therefore, the presence of saturation-level X-ray emission is an excellent indicator (though not guarantor) of youth.

Accordingly, X-ray photometry was obtained from the ROSAT All-Sky Survey (RASS; Voges et al. 1999, 2000) for every available star on the CTIOPI parallax program; the resulting systems have X-ray detections with better than $25 \%$ errors on the counts and are within $25^{\prime \prime}$ (95\% detection radius; Voges et al. 1999) of the proper-motion-corrected epoch 1991, equinox J2000 coordinates (close to the mean epoch of RASS). The calculation of $\log \left(L_{X} / L_{\text {bol }}\right)$ is taken from Schmitt et al. (1995), using bolometric calculations from Casagrande et al.

\footnotetext{
14 A complete table of all published parallaxes is available here: http://www.recons.org/
} 
Table 2

Photometric Results

\begin{tabular}{|c|c|c|c|c|c|c|c|c|c|c|c|c|c|c|c|c|c|}
\hline \\
\hline & Alternate & & & & No. of Phot. & & $\sigma \sigma$ & No. of Rel. & No. of & $\overline{J J}$ & ${ }_{H}$ & 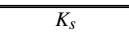 & Spectral & & Phot. & No. of & \\
\hline $\begin{array}{l}\text { Name } \\
\text { (1) }\end{array}$ & $\begin{array}{c}\text { Name } \\
\text { (2) }\end{array}$ & $\begin{array}{l}V_{J} \\
(3)\end{array}$ & $\begin{array}{c}R_{\mathrm{KC}} \\
(4)\end{array}$ & $\begin{array}{l}I_{\mathrm{KC}} \\
(5)\end{array}$ & $\begin{array}{c}\text { Observations } \\
\text { (6) }\end{array}$ & $\begin{array}{c}\pi \text { Filter } \\
(7)\end{array}$ & $\begin{array}{c}(\mathrm{mag}) \\
(8)\end{array}$ & $\begin{array}{c}\text { Nights } \\
\text { (9) }\end{array}$ & $\begin{array}{c}\text { Frames } \\
(10)\end{array}$ & $\begin{array}{c}\text { (2MASS) } \\
\text { (11) }\end{array}$ & $\begin{array}{c}\text { (2MASS) } \\
(12)\end{array}$ & $\begin{array}{c}\text { (2MASS) } \\
\text { (13) }\end{array}$ & $\begin{array}{l}\text { Type }^{\mathrm{a}} \\
\text { (14) }\end{array}$ & $\begin{array}{l}\text { Ref } \\
(15)\end{array}$ & $\begin{array}{l}\text { Dist. (pc) } \\
\text { (16) }\end{array}$ & $\begin{array}{l}\text { Relations } \\
\text { (17) }\end{array}$ & $\begin{array}{c}\text { Notes } \\
(18)\end{array}$ \\
\hline NLTT 372 & LP 404-32 & $15.87 \pm 0.04$ & $14.66 \pm 0.03$ & $13.12 \pm 0.05$ & 3 & V & 0.014 & 21 & 97 & $11.64 \pm 0.02$ & $11.00 \pm 0.03$ & $10.72 \pm 0.02$ & $\mathrm{M} 4.5 \mathrm{~V}$ & 1 & $47.39 \pm 7.47$ & 12 & 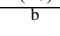 \\
\hline G $131-26 \mathrm{AB}$ & NLTT 375 & $13.52 \pm 0.06$ & $12.19 \pm 0.04$ & $10.50 \pm 0.04$ & 3 & V & 0.019 & 21 & 97 & $8.87 \pm 0.03$ & $8.26 \pm 0.03$ & $8.01 \pm 0.02$ & M4.0VeJ & 2 & $10.74 \pm 1.67$ & 12 & b \\
\hline SCR 0017-6645 & RBS 38 & $12.45 \pm 0.02$ & $11.37 \pm 0.01$ & $10.00 \pm 0.01$ & 2 & V & 0.035 & 11 & 54 & $8.56 \pm 0.02$ & $7.93 \pm 0.04$ & $7.70 \pm 0.02$ & $\mathrm{M} 2.5 \mathrm{Ve}$ & 2 & $14.82 \pm 2.69$ & 12 & \\
\hline GJ 2006A & RBS 67 & $12.95 \pm 0.02$ & $11.79 \pm 0.03$ & $10.29 \pm 0.03$ & 3 & V & 0.077 & 17 & 69 & $8.88 \pm 0.03$ & $8.24 \pm 0.04$ & $8.01 \pm 0.03$ & $\mathrm{M} 3.5 \mathrm{Ve}$ & 2 & $15.39 \pm 2.41$ & 12 & b \\
\hline GJ 2006B & & $13.25 \pm 0.03$ & $12.04 \pm 0.02$ & $10.48 \pm 0.02$ & 4 & V & 0.036 & 17 & 69 & $8.97 \pm 0.03$ & $8.39 \pm 0.03$ & $8.12 \pm 0.03$ & $\mathrm{M} 3.5 \mathrm{Ve}$ & 2 & $14.29 \pm 2.19$ & 12 & b \\
\hline SCR $0103-5515 \mathrm{ABC}$ & & $15.48 \pm 0.03$ & $14.00 \pm 0.02$ & $12.07 \pm 0.02$ & 2 & $R$ & 0.021 & 11 & 49 & $10.16 \pm 0.02$ & $9.58 \pm 0.03$ & $9.24 \pm 0.02$ & $\mathrm{M} 4.5 \mathrm{Ve}$ & 2 & $12.86 \pm 1.97$ & 12 & \\
\hline LP 467-16ABC & & $14.46 \pm 0.05$ & $12.95 \pm 0.01$ & $11.03 \pm 0.02$ & 2 & $R$ & 0.019 & 15 & 79 & $9.08 \pm 0.03$ & $8.51 \pm 0.04$ & $8.21 \pm 0.03$ & M $4.5 \mathrm{VeJ}$ & 2 & $7.88 \pm 1.22$ & 12 & \\
\hline GJ 2022AC & G 269-153AB & $13.56 \pm 0.04$ & $12.26 \pm 0.01$ & $10.62 \pm 0.05$ & 3 & $R$ & 0.067 & 14 & 31 & $9.20 \pm 0.04$ & $8.66 \pm 0.05$ & $8.24 \pm 0.03$ & $\mathrm{M} 4.0 \mathrm{VeJ}$ & 2 & $14.78 \pm 3.01$ & 12 & \\
\hline GJ 2022B & G 269-153C & $15.50 \pm 0.06$ & $14.09 \pm 0.02$ & $12.33 \pm 0.06$ & 3 & $R$ & 0.016 & 16 & 66 & $10.56 \pm 0.02$ & $10.01 \pm 0.02$ & $9.68 \pm 0.02$ & $\mathrm{M} 4.5 \mathrm{Ve}$ & 2 & $19.65 \pm 3.08$ & 12 & \\
\hline LP 993-115 & & $12.38 \pm 0.08$ & $11.17 \pm 0.03$ & $9.61 \pm 0.03$ & 3 & V & 0.021 & 15 & 71 & $8.14 \pm 0.02$ & $7.55 \pm 0.04$ & $7.27 \pm 0.02$ & M3.5Ve & 2 & $9.94 \pm 1.53$ & 12 & \\
\hline LP 993-116AB & RBS 353 & $12.69 \pm 0.06$ & $11.37 \pm 0.02$ & $9.67 \pm 0.02$ & 3 & V & 0.015 & 15 & 71 & $8.06 \pm 0.02$ & $7.53 \pm 0.04$ & $7.20 \pm 0.02$ & M4.0VeJ & 2 & $7.62 \pm 1.21$ & 12 & \\
\hline G 7-34 & & $13.84 \pm 0.02$ & $12.50 \pm 0.01$ & $10.75 \pm 0.01$ & 3 & $R$ & 0.022 & 15 & 72 & $9.03 \pm 0.03$ & $8.48 \pm 0.03$ & $8.18 \pm 0.02$ & M4.0Ve & 2 & $10.65 \pm 1.64$ & 12 & \\
\hline G 39-29AB & & $12.56 \pm 0.05$ & $11.31 \pm 0.01$ & $9.70 \pm 0.02$ & 3 & V & 0.015 & 13 & 70 & $8.17 \pm 0.02$ & $7.62 \pm 0.02$ & $7.33 \pm 0.02$ & $\mathrm{M} 4.0 \mathrm{VeJ}$ & 2 & $9.40 \pm 1.46$ & 12 & \\
\hline LP 655-48 & & $17.79 \pm 0.06$ & $15.72 \pm 0.03$ & $13.36 \pm 0.04$ & 4 & $I$ & 0.013 & 21 & 101 & $10.66 \pm 0.02$ & $9.99 \pm 0.02$ & $9.55 \pm 0.02$ & M6.5Ve & 2 & $8.45 \pm 1.33$ & 12 & \\
\hline LP 476-207ABC & HIP 23418 & $11.53 \pm 0.07$ & $10.33 \pm 0.04$ & $8.74 \pm 0.04$ & 2 & V & 0.021 & 12 & 64 & $7.21 \pm 0.02$ & $6.66 \pm 0.03$ & $6.37 \pm 0.02$ & M3.0VeJ & 2 & $6.28 \pm 0.97$ & 12 & \\
\hline BD- $21^{\circ} 1074 \mathrm{BC}$ & & $11.08 \pm 0.05$ & $9.92 \pm 0.05$ & $8.45 \pm 0.04$ & 4 & v & 0.046 & 16 & 95 & $7.00 \pm 0.02$ & $6.39 \pm 0.02$ & $6.11 \pm 0.02$ & M3.0VeJ & 2 & $6.37 \pm 1.00$ & 12 & b \\
\hline BD- $21^{\circ} 1074 \mathrm{~A}$ & RBS 620 & $10.41 \pm 0.02$ & $9.40 \pm 0.02$ & $8.25 \pm 0.02$ & 3 & V & 0.043 & 10 & 49 & $7.05 \pm 0.02$ & $6.39 \pm 0.05$ & $6.12 \pm 0.02$ & M1.5Ve & 2 & $9.91 \pm 1.72$ & 12 & b \\
\hline L 449-1AB & RBS 636 & $11.69 \pm 0.01$ & $10.48 \pm 0.02$ & $8.91 \pm 0.01$ & 3 & V & 0.024 & 17 & 87 & $7.40 \pm 0.03$ & $6.85 \pm 0.05$ & $6.56 \pm 0.02$ & M3.5 VeJ & 2 & $7.01 \pm 1.08$ & 12 & b \\
\hline SCR 0529-3239 & & $13.79 \pm 0.02$ & $12.50 \pm 0.01$ & $10.80 \pm 0.01$ & 2 & $R$ & 0.014 & 11 & 49 & $9.22 \pm 0.03$ & $8.61 \pm 0.04$ & $8.32 \pm 0.03$ & M4.0Ve & 2 & $12.83 \pm 1.98$ & 12 & \\
\hline SCR 0613-2742AB & & $12.30 \pm 0.03$ & $11.10 \pm 0.03$ & $9.55 \pm 0.02$ & 4 & V & 0.037 & 14 & 77 & $8.00 \pm 0.03$ & $7.43 \pm 0.07$ & $7.15 \pm 0.02$ & M4.0 VeJ & 2 & $8.98 \pm 1.41$ & 12 & \\
\hline L $34-26$ & & $11.31 \pm 0.03$ & $10.19 \pm 0.03$ & $8.79 \pm 0.03$ & 3 & V & 0.018 & 19 & 95 & $7.41 \pm 0.02$ & $6.86 \pm 0.03$ & $6.58 \pm 0.02$ & $\mathrm{M} 3.0 \mathrm{Ve}$ & 2 & $9.16 \pm 1.41$ & 12 & $\mathrm{~b}$ \\
\hline SCR 0757-7114 & & $12.45 \pm 0.03$ & $11.28 \pm 0.03$ & $9.77 \pm 0.03$ & 3 & V & 0.007 & 7 & 41 & $8.32 \pm 0.02$ & $7.75 \pm 0.04$ & $7.42 \pm 0.02$ & M3.5V & 2 & $11.36 \pm 1.76$ & 12 & \\
\hline SCR 1012-3124AB & & $13.51 \pm 0.02$ & $12.20 \pm 0.01$ & $10.51 \pm 0.03$ & 2 & V & 0.015 & 11 & 59 & $8.85 \pm 0.02$ & $8.26 \pm 0.05$ & $7.99 \pm 0.03$ & M4.0VeJ & 2 & $10.56 \pm 1.63$ & 12 & \\
\hline TWA 8B & & $15.22 \pm 0.07$ & $13.68 \pm 0.02$ & $11.76 \pm 0.03$ & 3 & v & 0.124 & 11 & 65 & $9.84 \pm 0.02$ & $9.28 \pm 0.02$ & $9.01 \pm 0.03$ & M5.0Ve & 2 & $11.60 \pm 1.87$ & 12 & \\
\hline TWA 8A & RBS 994 & $12.23 \pm 0.06$ & $11.14 \pm 0.04$ & $9.79 \pm 0.03$ & 3 & V & 0.078 & 11 & 65 & $8.34 \pm 0.02$ & $7.66 \pm 0.04$ & $7.43 \pm 0.02$ & M4.0Ve & 2 & $12.74 \pm 2.54$ & 12 & \\
\hline SCR 1214-2345 & & $13.96 \pm 0.02$ & $12.57 \pm 0.02$ & $10.78 \pm 0.03$ & 2 & v & 0.010 & 11 & 54 & $9.07 \pm 0.02$ & $8.56 \pm 0.05$ & $8.23 \pm 0.03$ & M4.0Ve & 2 & $10.66 \pm 1.75$ & 12 & \\
\hline G $165-8 \mathrm{AB}$ & RBS 1280 & $12.02 \pm 0.09$ & $10.77 \pm 0.05$ & $9.15 \pm 0.07$ & 3 & $R$ & 0.018 & 31 & 181 & $7.56 \pm 0.02$ & $7.00 \pm 0.02$ & $6.72 \pm 0.02$ & M4.0VeJ & 2 & $6.73 \pm 1.03$ & 12 & \\
\hline SCR 1425-4113AB & & $12.54 \pm 0.15$ & $11.48 \pm 0.17$ & $10.06 \pm 0.11$ & 2 & V & 0.078 & 11 & 57 & $8.55 \pm 0.03$ & $7.91 \pm 0.03$ & $7.61 \pm 0.02$ & M2.5VeJ & 2 & $12.95 \pm 2.89$ & 12 & \\
\hline GJ 1224 & L 920-26 & $13.48 \pm 0.04$ & $12.08 \pm 0.02$ & $10.31 \pm 0.03$ & 2 & $I$ & 0.013 & 25 & 170 & $8.64 \pm 0.02$ & $8.09 \pm 0.04$ & $7.83 \pm 0.03$ & $\mathrm{M} 4.0 \mathrm{Ve}$ & 2 & $9.11 \pm 1.55$ & 12 & \\
\hline G $141-29$ & LP 510-15 & $12.86 \pm 0.04$ & $11.58 \pm 0.04$ & $9.95 \pm 0.04$ & 3 & I & 0.013 & 17 & 82 & $8.36 \pm 0.02$ & $7.81 \pm 0.04$ & $7.55 \pm 0.02$ & M4.0Ve & 2 & $9.78 \pm 1.53$ & 12 & \\
\hline SCR 1942-2045 & & $14.33 \pm 0.05$ & $12.98 \pm 0.02$ & $11.25 \pm 0.01$ & 2 & $R$ & 0.019 & 9 & 48 & $9.60 \pm 0.02$ & $9.03 \pm 0.02$ & $8.76 \pm 0.02$ & M4.0Ve & 2 & $14.62 \pm 2.31$ & 12 & \\
\hline 2MASS 2009-0113 & & $14.47 \pm 0.05$ & $12.98 \pm 0.03$ & $11.16 \pm 0.03$ & 3 & $I$ & 0.015 & 14 & 71 & $9.40 \pm 0.03$ & $8.83 \pm 0.03$ & $8.51 \pm 0.02$ & $\mathrm{M} 4.5 \mathrm{Ve}$ & 2 & $10.81 \pm 1.79$ & 12 & \\
\hline SCR 2010-2801AB & & $12.98 \pm 0.03$ & $11.78 \pm 0.02$ & $10.20 \pm 0.02$ & 3 & $R$ & 0.012 & 11 & 57 & $8.65 \pm 0.02$ & $8.01 \pm 0.05$ & $7.73 \pm 0.03$ & M4.0 VeJ & 2 & $11.18 \pm 1.83$ & 12 & \\
\hline LEHPM2-0783 & SIP 2019-5816 & $17.17 \pm 0.04$ & $15.28 \pm 0.04$ & $13.03 \pm 0.02$ & 3 & I & 0.025 & 11 & 58 & $10.66 \pm 0.02$ & $10.10 \pm 0.03$ & $9.72 \pm 0.02$ & M6.5V Ve & 2 & $10.57 \pm 1.65$ & 12 & \\
\hline L 755-19 & & $12.47 \pm 0.02$ & $11.31 \pm 0.01$ & $9.81 \pm 0.02$ & 2 & $R$ & 0.018 & 11 & 56 & $8.39 \pm 0.03$ & $7.76 \pm 0.03$ & $7.50 \pm 0.03$ & M3.0Ve & 2 & $12.01 \pm 1.88$ & 12 & \\
\hline SCR 2033-2556 & & $14.87 \pm 0.02$ & $13.44 \pm 0.02$ & $11.57 \pm 0.01$ & 2 & $R$ & 0.017 & 11 & 48 & $9.71 \pm 0.02$ & $9.15 \pm 0.02$ & $8.88 \pm 0.02$ & $\mathrm{M} 4.5 \mathrm{Ve}$ & 2 & $12.04 \pm 1.88$ & 12 & \\
\hline SCR 2036-3607 & RBS 1687 & $11.66 \pm 0.03$ & $10.59 \pm 0.01$ & $9.27 \pm 0.02$ & 2 & V & 0.021 & 9 & 53 & $8.03 \pm 0.02$ & $7.42 \pm 0.03$ & $7.17 \pm 0.02$ & M2.5Ve & 2 & $14.18 \pm 2.22$ & 12 & \\
\hline GJ 799A & AT Mic A & $10.36 \pm 0.03$ & $9.08 \pm 0.03$ & $7.40 \pm 0.03$ & 3 & v & 0.064 & 18 & 131 & $5.81 \pm 0.03$ & $5.20 \pm 0.05$ & $4.94 \pm 0.04$ & M4.0VeJ & 2 & $2.77 \pm 0.43$ & 12 & $\mathrm{~b}$ \\
\hline GJ 799B & AT Mic B & & & $\ldots$ & & V & 0.076 & 18 & 131 & & & & & & & $\ldots$ & $b, c$ \\
\hline LHS 3799 & L 788-34 & $13.30 \pm 0.03$ & $11.87 \pm 0.02$ & $10.04 \pm 0.03$ & 5 & V & 0.014 & 21 & 118 & $8.24 \pm 0.03$ & $7.64 \pm 0.05$ & $7.32 \pm 0.02$ & $\mathrm{M} 4.5 \mathrm{Ve}$ & 2 & $6.07 \pm 0.93$ & 12 & $\mathrm{~b}$ \\
\hline GJ $1284 \mathrm{AB}$ & RBS 2013 & $11.14 \pm 0.04$ & $10.02 \pm 0.04$ & $8.59 \pm 0.04$ & 3 & V & 0.027 & 20 & 95 & $7.20 \pm 0.02$ & $6.61 \pm 0.04$ & $6.33 \pm 0.03$ & M3.0Ve & 2 & $7.78 \pm 1.21$ & 12 & $\mathrm{~b}$ \\
\hline \multicolumn{18}{|c|}{ Previously Published } \\
\hline$\overline{\text { LHS } 1302}$ & G 159-3 & $14.49 \pm 0.05$ & $13.00 \pm 0.02$ & $11.16 \pm 0.03$ & 5 & $R$ & 0.021 & 26 & 141 & $9.41 \pm 0.02$ & $8.84 \pm 0.02$ & $8.55 \pm 0.02$ & $\mathrm{M} 4.5 \mathrm{Ve}$ & 2 & $11.04 \pm 1.88$ & 12 & \\
\hline LHS 1358 & G $159-46$ & $13.58 \pm 0.03$ & $12.31 \pm 0.03$ & $10.66 \pm 0.02$ & 2 & $R$ & 0.015 & 11 & 58 & $9.06 \pm 0.03$ & $8.52 \pm 0.03$ & $8.17 \pm 0.02$ & M4.0V & 1 & $12.54 \pm 1.94$ & 12 & \\
\hline G 99-49 & & $11.31 \pm 0.05$ & $10.04 \pm 0.04$ & $8.43 \pm 0.03$ & 5 & V & 0.017 & 23 & 145 & $6.91 \pm 0.02$ & $6.31 \pm 0.02$ & $6.04 \pm 0.02$ & $\mathrm{M} 3.5 \mathrm{Ve}$ & 2 & $5.09 \pm 0.79$ & 12 & b \\
\hline $\mathrm{APCol}$ & LP 949-15 & $12.96 \pm 0.02$ & $11.49 \pm 0.02$ & $9.60 \pm 0.02$ & 4 & v & 0.017 & 26 & 158 & $7.74 \pm 0.03$ & $7.18 \pm 0.02$ & $6.87 \pm 0.02$ & $\mathrm{M} 4.5 \mathrm{Ve}$ & 2 & $4.62 \pm 0.72$ & 12 & $\mathrm{~b}$ \\
\hline G 41-14ABC & LHS 6158 & $10.92 \pm 0.04$ & $9.67 \pm 0.02$ & $8.05 \pm 0.02$ & 3 & V & 0.013 & 22 & 159 & $6.51 \pm 0.02$ & $5.97 \pm 0.03$ & $5.69 \pm 0.02$ & M3.5 VeJ & 2 & $4.39 \pm 0.69$ & 12 & $\mathrm{~b}$ \\
\hline TWA $27 \mathrm{AB}$ & 2MA 1207-3932 & $19.95 \pm 0.20$ & $17.99 \pm 0.07$ & $15.92 \pm 0.05$ & 5 & I & 0.015 & 12 & 54 & $13.00 \pm 0.03$ & $12.39 \pm 0.03$ & $11.95 \pm 0.03$ & M8 J & 3 & $26.99 \pm 5.15$ & 12 & \\
\hline LHS 2729 & L 617-35 & $12.89 \pm 0.06$ & $11.68 \pm 0.03$ & $10.14 \pm 0.02$ & 2 & $R$ & 0.012 & 9 & 56 & $8.66 \pm 0.03$ & $8.07 \pm 0.06$ & $7.78 \pm 0.03$ & $\mathrm{M} 3.5 \mathrm{Ve}$ & 2 & $12.59 \pm 1.94$ & 12 & \\
\hline LHS 2836 & L $763-63$ & $12.88 \pm 0.04$ & $11.60 \pm 0.02$ & $9.90 \pm 0.03$ & 3 & V & 0.013 & 22 & 108 & $8.33 \pm 0.04$ & $7.76 \pm 0.06$ & $7.45 \pm 0.03$ & M4.0Ve & 2 & $8.86 \pm 1.39$ & 12 & \\
\hline GJ 1207 & LHS 3255 & $12.25 \pm 0.04$ & $10.99 \pm 0.05$ & $9.43 \pm 0.05$ & 5 & V & 0.199 & 27 & 124 & $7.97 \pm 0.02$ & $7.44 \pm 0.08$ & $7.12 \pm 0.02$ & $\mathrm{M} 3.5 \mathrm{Ve}$ & 2 & $9.36 \pm 1.59$ & 12 & b \\
\hline LHS 4016AB & L 649-24 & $12.34 \pm 0.04$ & $11.25 \pm 0.03$ & $9.90 \pm 0.02$ & 3 & V & 0.014 & 16 & 68 & $8.58 \pm 0.03$ & $8.02 \pm 0.05$ & $7.74 \pm 0.02$ & M2.5 $\mathrm{VeJ}$ & 2 & $17.18 \pm 2.65$ & 12 & \\
\hline
\end{tabular}

Notes. Photometry data collected on the sample.

(2007). "J" indicates joint spectral types from unresolved multiples.

No independent photometry. 


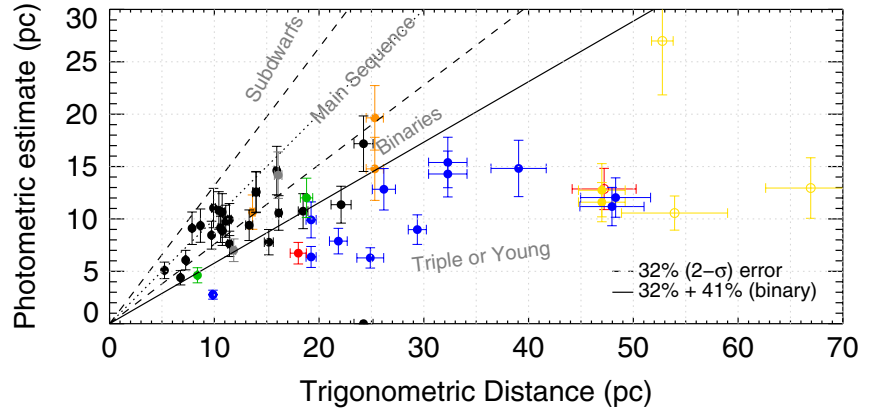

Figure 1. Diagram of the 51 resolved components of the 45 systems in this paper, demonstrating their overluminosity in terms of their trigonometric distances $(X$-axis) and photometric distances ( $Y$-axis). Objects are color-coded by the association to which they are ultimately linked as members: TW Hydrae are shown in yellow; $\beta$ Pictoris in blue; Tucana-Horologium, Columba, and Carina in red; Argus in green; AB Doradus in orange; and Castor and Ursa Major in gray. Open circles have no RASS detection.

(A color version of this figure is available in the online journal.)

(2008). We found positive evidence of saturated X-ray emission for 39 of our star systems, many of which are also overluminous.

The remaining six star systems came to our attention purely by the overluminosity criterion. They exhibit no X-ray emission in the ROSAT catalogs, but their luminosity makes it difficult to explain them as unresolved binaries or triples (Figure 1).

Among the 45 systems considered here, we have individual photometry and astrometry of 51 components, ${ }^{15}$ because six of our star systems contain binaries with separations more than 1 arcsec. Many of the stars in this paper were originally identified as active by Riaz et al. (2006), and several have already been identified as young by Zuckerman \& Song (2004), Shkolnik et al. (2012), and Malo et al. (2013). Ten systems were published in previous papers in this series; their astrometry and photometry is reprinted from the earlier papers without change.

\section{OBSERVATIONS AND REDUCTIONS}

\subsection{Photometry}

All CTIOPI photometry is conducted with the CTIO $0.9 \mathrm{~m}$ telescope, initially (1999-2003) under the NOAO Survey Programs grant; later (2003-present) via the SMARTS Consortium. Photometry is conducted in three filters (Tektronix 2 VRI), utilizing only the central quarter $\left(6\right.$ '.8 $\times 6$ ' $.8 \mathrm{FOV}, 401$ mas pixel $\left.^{-1}\right)$ of the Tektronics $2046 \times 2046$ CCD to minimize distortions for astrometry. These values are then transformed to standard $V_{\mathrm{J}} R_{\mathrm{KC}} I_{\mathrm{KC}}{ }^{16}$ (hereafter without subscripts) photometry using observations of standards from Graham (1982), Landolt (1992), and Landolt (2007). The resulting photometry can be found in Table 2. Further details of the observation and reduction procedures can be found in Jao et al. (2005) and Winters et al. (2011). The photometric errors quoted in Columns 3, 4, and 5 of Table 2 combine the Poisson errors, errors on the nightly calibration fit, and standard deviation of multiple nights of photometry (see column 6). Generally, the latter is the greatest contributor to the collective error, particularly when the star is active, as these stars are. This VRI photometry, along with Two Micron All Sky Survey (2MASS) JHK photometry (Skrutskie et al. 2006),

\footnotetext{
15 As seen in Tables 2 and 3, GJ 2022AC was observed for standard photometry but not astrometry, and GJ 799B has resolved astrometry but not photometry.

16 Subscripts: "J" indicates Johnson, and "KC" indicates Kron-Cousins (SAAO system), which is more often known as Cousins. The central wavelengths for $V_{\mathrm{J}}, R_{\mathrm{KC}}$, and $I_{\mathrm{KC}}$ are 5475,6425 , and $8075 \AA$, respectively.
}

is printed in Columns 11, 12, and 13. The measured colors were used to estimate absolute $K$ magnitudes based on the 12 color-magnitude relations presented by Henry et al. (2004). The photometric distances presented in Column 16 of Table 2 were derived from the mean of the distance moduli implied by the absolute $K$ magnitudes and the 2MASS apparent $K$ magnitude.

Relative photometry (for variability studies) comes from our parallax pipeline. With multiple nights of data in the filter used for parallax, we use the methods in Honeycutt (1992) to derive the nightly offsets and zero points for relative instrumental photometry (Jao et al. 2008) to derive stellar variability. These values are given in Column 8 of Table 2 .

\subsection{Astrometry}

CTIOPI astrometry is carried out using the same telescope and camera configuration as that used for photometry (Section 3.1) but uses only one filter for each object, chosen to provide the best balance between target(s) and reference star signal-to-noise ratio values. The astrometric pipeline uses all available images taken at hour angles less than two hours, and produces parallaxes, proper motions, and time-series photometry in the parallax filter, all relative to between 5 and 15 "reference" stars within a few arcminutes of the target stars and visible in our images. Parallaxes were corrected to absolute values (Columns 11 and 12 of Table 3) using the mean of the photometric distances to the reference stars, with a typical correction of $1.5 \pm 0.5$ mas. For a small number of targets with seemingly nearby reference fields (mean photometric parallax estimate, $>3.0$ mas), we assume the reference stars are actually reddened by some galactic source, and instead apply the typical correction stated above. Between 2005 March and 2009 September, a different $V$-band filter was used for astrometric and photometric observations. While photometrically identical to the original $V$ filter, it exhibited slightly inferior astrometric performance (Riedel et al. 2010), and all astrometric solutions that incorporate data from it are marked as such in Column 16 of Table 3. Additional details of CTIOPI observing procedures can be found in Jao et al. (2005), Henry et al. (2006), and other papers in this series.

\subsection{Interferometry}

Four of the objects in this paper-BD- $21^{\circ} 1074 \mathrm{BC}, \mathrm{SCR}$ 0613-2742AB, L 449-1AB, and SCR 2010-2801AB-were selected for their X-ray brightness and observed with the Hubble Space Telescope's (HST's) Fine Guidance Sensors (FGSs) in Cycle 16B, in proposal 11943/11944 ("Binaries at the Extremes of the H-R Diagram") using the F583W filter ${ }^{17}$ with no pupil. Reductions were carried out for both axes, providing submilliarcsecond precision separations, and delta magnitudes (hereafter $\Delta \mathrm{mag}$ ). All four targets were found to be binaries (see Section 5.1 below) and their separations, position angles, and magnitude differences were determined by fitting with singlestar fringe scans as described by Nelan et al. (2004).

Hubble's FGSs are implemented as three movable units equipped with Koesters prisms, which allow them to function as a two-dimensional interferometer. Two FGS units lock on guide stars and stabilize the spacecraft, while the third (FGS 1r) scans back and forth across the star. Rather than a Michelson interferometer, light is channeled through a linear polarizing beamsplitter, and then through the unit's Koesters prisms, where

\footnotetext{
17 The bandpass of the F583W filter is shown here: http://www.stsci.edu/hst/fgs/design/filters (checked 2013 June 4).
} 
Table 3

Astrometric Results

\begin{tabular}{|c|c|c|c|c|c|c|c|c|c|c|c|c|c|c|c|}
\hline $\begin{array}{l}\text { Name } \\
\text { (1) }\end{array}$ & $\begin{array}{ll}\text { R.A. } & \\
& \text { (J }\end{array}$ & $\begin{array}{ll} & \text { Decl. } \\
0)^{\mathrm{a}} & \text { (3) }\end{array}$ & $\begin{array}{l}\text { Filter } \\
\text { (4) }\end{array}$ & $\begin{array}{c}N_{\text {sea }} \mathrm{b} \\
(5)\end{array}$ & $\begin{array}{c}N_{\mathrm{frm}} \\
(6)\end{array}$ & $\begin{array}{c}\text { Coverage }{ }^{b} \\
\text { (7) }\end{array}$ & $\begin{array}{c}\text { Years }^{b} \\
(8)\end{array}$ & $\begin{array}{c}N_{\text {ref }} \\
\text { (9) }\end{array}$ & $\begin{array}{c}\pi(\mathrm{Rel}) \\
(\mathrm{mas}) \\
(10)\end{array}$ & $\begin{array}{c}\pi \text { (Corr) } \\
\text { (mas) } \\
(11)\end{array}$ & $\begin{array}{c}\pi(\mathrm{Abs}) \\
(\mathrm{mas}) \\
(12)\end{array}$ & $\begin{array}{c}\mu \\
\left(\operatorname{mas~yr}^{-1}\right) \\
(13)\end{array}$ & $\begin{array}{l}\text { P.A. } \\
\text { (deg) } \\
(14)\end{array}$ & $\begin{array}{c}V_{\tan } \\
\left(\mathrm{km} \mathrm{s}^{-1}\right) \\
(15)\end{array}$ & $\begin{array}{c}\text { Notes } \\
(16)\end{array}$ \\
\hline NLTT 372 & 000851.79 & +204907.9 & $V$ & $11 \mathrm{~s}$ & 97 & $1999.71-2011.89$ & 12.18 & 7 & $10.50 \pm 1.44$ & $1.16 \pm 0.07$ & $11.66 \pm 1.44$ & $147.8 \pm 0.3$ & $121.1 \pm 0.23$ & 60.1 & 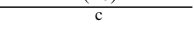 \\
\hline G 131-26AB & 000853.92 & +205025.4 & V & $11 \mathrm{~s}$ & 97 & 1999.71-2011.89 & 12.18 & 7 & $52.97 \pm 1.35$ & $1.16 \pm 0.07$ & $54.13 \pm 1.35$ & $251.2 \pm 0.3$ & $194.4 \pm 0.11$ & 22.0 & c \\
\hline SCR 0017-6645 & 001723.53 & -664512.5 & V & $4 \mathrm{~s}$ & 54 & $2009.75-2012.88$ & 3.13 & 9 & $24.71 \pm 1.73$ & $0.90 \pm 0.10$ & $25.61 \pm 1.73$ & $93.6 \pm 1.8$ & $101.1 \pm 1.82$ & 17.3 & \\
\hline GJ 2006A & 002750.24 & -323306.1 & V & $9 \mathrm{~s}$ & 69 & $2000.57-2010.82$ & 10.25 & 7 & $29.49 \pm 2.50$ & $0.65 \pm 0.11$ & $30.14 \pm 2.50$ & $115.0 \pm 0.6$ & $109.6 \pm 0.55$ & 18.1 & c \\
\hline GJ 2006B & 002750.36 & -323323.9 & V & $9 \mathrm{~s}$ & 69 & $2000.57-2010.82$ & 10.25 & 7 & $31.13 \pm 2.47$ & $0.65 \pm 0.11$ & $31.78 \pm 2.47$ & $115.8 \pm 0.6$ & $108.6 \pm 0.53$ & 17.3 & c \\
\hline SCR 0103-5515ABC & 010335.63 & -551556.2 & $R$ & $6 \mathrm{~s}$ & 49 & 2007.82-2012.87 & 5.05 & 8 & $20.77 \pm 1.37$ & $0.41 \pm 0.07$ & $21.18 \pm 1.37$ & $89.0 \pm 1.0$ & $112.0 \pm 1.24$ & 19.9 & \\
\hline LP 467-16AB & 011125.41 & +152621.6 & $R$ & $8 \mathrm{~s}$ & 79 & 1999.71-2009.57 & 9.86 & 7 & $44.88 \pm 1.77$ & $0.91 \pm 0.16$ & $45.79 \pm 1.78$ & $222.9 \pm 0.6$ & $122.7 \pm 0.29$ & 23.1 & \\
\hline GJ $2022 \mathrm{AC}$ & 012430.62 & -335501.6 & & $\ldots$ & & & $\ldots$ & $\ldots$ & & & & & & & d \\
\hline GJ 2022B & 012430.62 & -335501.6 & $R$ & $7 \mathrm{~s}$ & 66 & 1999.62-2011.53 & 11.91 & 7 & $38.40 \pm 2.13$ & $0.40 \pm 0.07$ & $38.80 \pm 2.13$ & $206.0 \pm 0.7$ & $127.5 \pm 0.36$ & 25.2 & \\
\hline LP 993-115 & 024510.71 & -434432.4 & $V$ & $8 \mathrm{~s}$ & 71 & 1999.62-2012.95 & 13.32 & 7 & $88.10 \pm 1.73$ & $1.52 \pm 0.10$ & $89.62 \pm 1.73$ & $388.5 \pm 0.3$ & $175.8 \pm 0.06$ & 20.5 & \\
\hline LP 993-116AB & 024514.32 & -434410.6 & V & $8 \mathrm{~s}$ & 71 & $1999.62-2012.95$ & 13.32 & 7 & $82.60 \pm 2.08$ & $1.52 \pm 0.10$ & $84.12 \pm 2.08$ & $367.6 \pm 0.4$ & $175.3 \pm 0.08$ & 20.7 & \\
\hline G 7-34 & 041718.52 & +084922.1 & $R$ & $6 \mathrm{~s}$ & 72 & 1999.64-2007.83 & 8.19 & 9 & $71.98 \pm 1.26$ & $1.29 \pm 0.19$ & $73.27 \pm 1.27$ & $395.2 \pm 0.7$ & $161.2 \pm 0.17$ & 25.6 & \\
\hline G 39-29AB & 043812.59 & +281300.0 & $V$ & $5 \mathrm{~s}$ & 70 & $2000.88-2005.06$ & 4.18 & 8 & $76.01 \pm 2.00$ & $2.60 \pm 0.40$ & $78.61 \pm 2.04$ & $403.2 \pm 1.8$ & $103.0 \pm 0.42$ & 24.3 & \\
\hline LP $655-48$ & 044023.28 & -053008.1 & $I$ & $10 \mathrm{~s}$ & 101 & 2003.95-2012.89 & 8.94 & 7 & $101.29 \pm 0.71$ & $1.32 \pm 0.08$ & $102.61 \pm 0.71$ & $359.4 \pm 0.2$ & $69.2 \pm 0.06$ & 16.6 & \\
\hline LP 476-207ABC & 050158.81 & +095858.8 & V & $5 \mathrm{~s}$ & 64 & $2000.06-2005.06$ & 5.00 & 8 & $39.52 \pm 2.11$ & $1.15 \pm 0.20$ & $40.67 \pm 2.12$ & $106.4 \pm 1.4$ & $165.4 \pm 1.31$ & 12.4 & \\
\hline BD- $21^{\circ} 1074 \mathrm{BC}$ & 050649.47 & -213503.8 & $V$ & $7 \mathrm{~s}$ & 95 & $1999.81-2012.16$ & 12.35 & 5 & $48.51 \pm 1.62$ & $2.02 \pm 0.12$ & $50.53 \pm 1.62$ & $50.7 \pm 0.4$ & $151.4 \pm 0.84$ & 4.8 & c \\
\hline $\mathrm{BD}-21^{\circ} 1074 \mathrm{~A}$ & 050649.92 & -213509.2 & V & $4 \mathrm{c}$ & 49 & $2000.06-2012.16$ & 12.10 & 5 & $52.54 \pm 2.16$ & $2.02 \pm 0.12$ & $54.56 \pm 2.16$ & $51.3 \pm 0.5$ & $111.1 \pm 1.06$ & 4.5 & c \\
\hline L 449-1AB & 051722.91 & -352154.7 & V & $6 c$ & 87 & 2007.81-2012.96 & 5.15 & 6 & $83.60 \pm 1.34$ & $0.78 \pm 0.12$ & $84.38 \pm 1.35$ & $280.5 \pm 0.8$ & $234.5 \pm 0.33$ & 15.8 & c \\
\hline SCR 0529-3239 & 052944.69 & -323914.2 & $R$ & $3 \mathrm{c}$ & 49 & $2008.85-2011.96$ & 3.10 & 6 & $37.08 \pm 1.60$ & $1.11 \pm 0.14$ & $38.19 \pm 1.61$ & $22.4 \pm 1.6$ & $82.7 \pm 6.37$ & 2.8 & \\
\hline SCR 0613-2742AB & 061313.31 & -274205.5 & V & $4 \mathrm{c}$ & 77 & 2009.93-2013.10 & 3.17 & 6 & $32.36 \pm 0.99$ & $1.68 \pm 0.17$ & $34.04 \pm 1.00$ & $11.2 \pm 1.0$ & $213.3 \pm 10.39$ & 1.6 & e \\
\hline L 34-26 & 074912.71 & -764206.6 & v & $8 \mathrm{~s}$ & 94 & $2006.21-2012.88$ & 6.68 & 7 & $92.86 \pm 2.05$ & $1.50 \pm 0.50$ & $94.36 \pm 2.11$ & $225.2 \pm 1.0$ & $206.6 \pm 0.46$ & 11.1 & cf \\
\hline SCR 0757-7114 & 075732.55 & -711453.8 & V & $3 \mathrm{c}$ & 41 & $2009.92-2011.96$ & 2.04 & 10 & $44.17 \pm 1.96$ & $1.08 \pm 0.12$ & $45.25 \pm 1.96$ & $104.1 \pm 2.5$ & $90.5 \pm 1.94$ & 10.9 & \\
\hline SCR 1012-3124AB & 101209.08 & -312445.2 & v & $3 \mathrm{c}$ & 59 & $2010.01-2012.27$ & 2.26 & 9 & $17.52 \pm 1.74$ & $1.02 \pm 0.12$ & $18.54 \pm 1.74$ & $70.5 \pm 1.9$ & $258.0 \pm 2.61$ & 18.0 & \\
\hline TWA 8B & 113241.17 & -265209.0 & V & $5 \mathrm{~s}$ & 65 & 2000.14-2011.16 & 11.02 & 6 & $19.90 \pm 1.44$ & $1.32 \pm 0.12$ & $21.22 \pm 1.44$ & $75.3 \pm 0.3$ & $253.9 \pm 0.42$ & 16.8 & \\
\hline TWA 8A & 113241.25 & -265155.9 & V & $5 \mathrm{~s}$ & 65 & 2000.14-2011.16 & 11.02 & 6 & $20.01 \pm 1.40$ & $1.32 \pm 0.12$ & $21.33 \pm 1.41$ & $76.6 \pm 0.3$ & $251.5 \pm 0.41$ & 17.0 & \\
\hline SCR 1214-2345 & 121408.67 & -234517.0 & V & $4 \mathrm{~s}$ & 54 & $2010.16-2013.39$ & 3.23 & 10 & $90.73 \pm 1.55$ & $0.66 \pm 0.09$ & $91.39 \pm 1.55$ & $99.6 \pm 1.2$ & $34.3 \pm 1.32$ & 5.2 & \\
\hline G $165-8 \mathrm{AB}$ & 133146.62 & +291636.6 & $R$ & $10 \mathrm{~s}$ & 181 & $2000.14-2009.25$ & 9.11 & 5 & $53.56 \pm 2.36$ & $1.95 \pm 0.31$ & $55.51 \pm 2.38$ & $260.1 \pm 1.1$ & $237.1 \pm 0.46$ & 22.2 & \\
\hline SCR $1425-4113 \mathrm{AB}$ & 142529.13 & -411332.4 & V & $3 c$ & 57 & 2010.16-2012.41 & 2.26 & 11 & $14.38 \pm 0.95$ & $0.56 \pm 0.13$ & $14.94 \pm 0.96$ & $59.4 \pm 1.4$ & $224.6 \pm 2.62$ & 18.8 & \\
\hline GJ 1224 & 180732.85 & -155747.0 & I & $10 \mathrm{~s}$ & 170 & 2003.52-2012.52 & 9.00 & 7 & $125.04 \pm 0.92$ & $1.50 \pm 0.50$ & $126.54 \pm 1.05$ & $702.3 \pm 0.4$ & $241.0 \pm 0.06$ & 26.1 & f \\
\hline G $141-29$ & 184244.99 & +1354 17.1 & I & $7 \mathrm{~s}$ & 82 & $2003.52-2012.58$ & 9.06 & 5 & $88.59 \pm 1.84$ & $1.50 \pm 0.50$ & $90.09 \pm 1.91$ & $360.5 \pm 0.8$ & $354.6 \pm 0.18$ & 18.3 & f \\
\hline SCR 1942-2045 & 194212.82 & -204548.0 & $R$ & $4 \mathrm{c}$ & 48 & 2010.66-2013.38 & 2.72 & 10 & $62.37 \pm 1.07$ & $1.00 \pm 0.18$ & $63.37 \pm 1.09$ & $144.7 \pm 1.4$ & $183.7 \pm 0.80$ & 10.8 & \\
\hline 2MASS 2009-0113 & 200918.24 & -011338.2 & $I$ & $8 \mathrm{~s}$ & 71 & 2004.73-2013.39 & 8.66 & 9 & $94.00 \pm 1.53$ & $1.95 \pm 0.17$ & $95.95 \pm 1.54$ & $371.1 \pm 0.6$ & $187.7 \pm 0.16$ & 18.3 & \\
\hline SCR $2010-2801 \mathrm{AB}$ & 201000.03 & -280141.2 & $R$ & $4 \mathrm{~s}$ & 57 & $2008.71-2011.62$ & 2.91 & 10 & $19.86 \pm 1.32$ & $0.99 \pm 0.12$ & $20.85 \pm 1.33$ & $74.9 \pm 1.2$ & $147.5 \pm 1.73$ & 17.0 & \\
\hline LEHPM2-0783 & 201949.82 & -581643.0 & I & $4 \mathrm{c}$ & 58 & $2006.37-2009.63$ & 3.26 & 9 & $61.24 \pm 1.02$ & $0.69 \pm 0.09$ & $61.93 \pm 1.02$ & $331.4 \pm 0.8$ & $185.4 \pm 0.22$ & 25.4 & \\
\hline L 755-19 & 202843.63 & -112830.8 & $R$ & $6 \mathrm{~s}$ & 56 & $2007.82-2012.42$ & 4.60 & 6 & $52.17 \pm 1.66$ & $1.01 \pm 0.15$ & $53.18 \pm 1.67$ & $185.2 \pm 1.1$ & $119.7 \pm 0.69$ & 16.5 & \\
\hline SCR 2033-2556 & 203337.59 & -255652.1 & $R$ & $5 \mathrm{~s}$ & 48 & 2008.71-2012.58 & 3.87 & 8 & $19.67 \pm 1.40$ & $1.03 \pm 0.29$ & $20.70 \pm 1.43$ & $86.3 \pm 1.2$ & $142.8 \pm 1.59$ & 19.8 & \\
\hline SCR 2036-3607 & 203608.30 & -360711.5 & V & $4 \mathrm{~s}$ & 53 & $2009.62-2012.42$ & 2.80 & 7 & $60.67 \pm 1.32$ & $1.46 \pm 0.48$ & $62.13 \pm 1.40$ & $51.4 \pm 1.6$ & $7.3 \pm 2.81$ & 3.9 & \\
\hline GJ 799A & 204151.14 & -322607.8 & V & $9 \mathrm{~s}$ & 131 & $2003.52-2012.58$ & 9.06 & 7 & $98.10 \pm 1.58$ & $2.72 \pm 0.14$ & $100.82 \pm 1.59$ & $449.9 \pm 0.6$ & $149.4 \pm 0.14$ & 21.2 & c \\
\hline GJ 799B & 204151.14 & -322607.8 & V & $9 \mathrm{~s}$ & 131 & 2003.52-2012.58 & 9.06 & 7 & $100.06 \pm 1.63$ & $2.72 \pm 0.14$ & $102.78 \pm 1.64$ & $433.4 \pm 0.6$ & $137.2 \pm 0.16$ & 20.0 & c \\
\hline LHS 3799 & 222307.00 & -173626.1 & V & $9 \mathrm{~s}$ & 118 & $2003.52-2012.70$ & 9.18 & 6 & $137.70 \pm 1.86$ & $0.47 \pm 0.14$ & $138.17 \pm 1.87$ & $769.1 \pm 0.4$ & $157.7 \pm 0.06$ & 26.4 & c \\
\hline GJ $1284 \mathrm{AB}$ & 233013.45 & -202327.4 & V & $8 \mathrm{~s}$ & 95 & $2003.51-2011.51$ & 8.00 & 5 & $66.35 \pm 2.27$ & $1.55 \pm 0.29$ & $67.90 \pm 2.29$ & $363.5 \pm 0.9$ & $121.1 \pm 0.27$ & 25.4 & c \\
\hline \multicolumn{16}{|c|}{ Examples } \\
\hline $\begin{array}{l}\text { LHS } 1302 \\
\end{array}$ & 015104.09 & -060705.0 & $R$ & $7 \mathrm{c}$ & 141 & 1999.75-2005.96 & 6.25 & 6 & $100.14 \pm 1.89$ & $0.64 \pm 0.06$ & $100.78 \pm 1.89$ & $597.1 \pm 0.9$ & $115.5 \pm 0.17$ & 28.1 & Henry et al. (2006) \\
\hline LHS 1358 & 021254.63 & +00 0016.8 & $R$ & $5 \mathrm{~s}$ & 58 & $1999.71-2003.86$ & 4.15 & 5 & $64.09 \pm 2.07$ & $1.18 \pm 0.13$ & $65.27 \pm 2.07$ & $558.3 \pm 1.3$ & $086.1 \pm 0.20$ & 40.5 & Riedel et al. (2010) \\
\hline G 99-49 & 060003.51 & +024223.6 & V & $7 \mathrm{c}$ & 145 & $1999.91-2005.96$ & 6.06 & 7 & $189.43 \pm 1.82$ & $1.50 \pm 0.50$ & $190.93 \pm 1.89$ & $312.5 \pm 0.8$ & $97.5 \pm 0.24$ & 7.8 & Henry et al. (2006) $\mathrm{c}, \mathrm{f}$ \\
\hline $\mathrm{APCol}$ & 060452.16 & -343336.0 & V & $7 \mathrm{c}$ & 158 & 2004.74-2011.23 & 6.48 & 14 & $118.26 \pm 0.97$ & $0.95 \pm 0.11$ & $119.21 \pm 0.98$ & $342.0 \pm 0.5$ & $4.6 \pm 0.13$ & 13.6 & Riedel et al. $(2011)^{\mathrm{C}}$ \\
\hline G 41-14ABC & 085856.33 & +082826.0 & V & $7 \mathrm{c}$ & 159 & 1999.97-2005.96 & 5.99 & 5 & $145.40 \pm 1.97$ & $2.26 \pm 0.22$ & $147.66 \pm 1.98$ & $502.7 \pm 0.9$ & $130.0 \pm 0.20$ & 16.1 & Henry et al. (2006) ${ }^{\mathrm{c}}$ \\
\hline TWA $27 \mathrm{AB}$ & 120733.46 & -393254.0 & I & $3 \mathrm{c}$ & 54 & 2005.41-2007.56 & 2.14 & 7 & $17.93 \pm 1.03$ & $0.58 \pm 0.05$ & $18.51 \pm 1.03$ & $66.7 \pm 1.5$ & $250.0 \pm 2.40$ & 17.1 & Gizis et al. (2007) \\
\hline LHS 2729 & 132338.02 & -255445.1 & $R$ & $5 \mathrm{~s}$ & 56 & $2001.15-2005.09$ & 3.94 & 12 & $70.32 \pm 1.52$ & $1.16 \pm 0.14$ & $71.48 \pm 1.53$ & $633.9 \pm 1.3$ & $249.8 \pm 0.21$ & 42.0 & Riedel et al. (2010) \\
\hline LHS 2836 & 135910.45 & -195003.4 & V & $3 c$ & 108 & 2000.14-2004.18 & 4.04 & 8 & $91.22 \pm 0.86$ & $1.64 \pm 0.23$ & $92.86 \pm 0.89$ & $573.4 \pm 1.0$ & $252.0 \pm 0.17$ & 29.3 & Riedel et al. (2010) \\
\hline GJ 1207 & 165705.73 & -042056.3 & V & $7 \mathrm{c}$ & 124 & $1999.62-2005.71$ & 6.09 & 10 & $113.36 \pm 1.44$ & $2.03 \pm 0.44$ & $115.39 \pm 1.51$ & $608.5 \pm 0.8$ & $127.1 \pm 0.15$ & 25.0 & Henry et al. $(2006)^{c}$ \\
\hline LHS 4016AB & 234836.06 & -273938.9 & $V$ & $6 \mathrm{~s}$ & 68 & $2000.87-2009.75$ & 8.87 & 6 & $40.75 \pm 1.54$ & $0.50 \pm 0.19$ & $41.25 \pm 1.55$ & $595.3 \pm 0.4$ & $246.2 \pm 0.08$ & 68.4 & Riedel et al. (2010) \\
\hline
\end{tabular}

${ }^{\text {a }}$ Coordinates are epoch and equinox 2000; each target's coordinates were extracted from 2MASS and then transformed to epoch 2000 using the proper motions and position angles listed here.

${ }^{c}$ Astrometric results include new $V$ filter data.

Not using independent astrometry (see Section 5).

The astrometric perturbation was incorporated into the final astrometric fit. 
the light interferes with itself, allowing two dimensions of fringes to be read out simultaneously.

As an interferometric instrument, FGS is capable of measuring single-axis separations as small as 8 mas, for objects as faint as $V=16.8$. It has been routinely used for orbital mapping and submilliarcsecond parallaxes (e.g., Franz et al. 1998; Benedict et al. 1999; McArthur et al. 2011).

\subsection{Spectroscopy 3.4.1. CTIO 1.5 m RCSpec}

Spectroscopic observations of all the resolved objects in this paper (except SCR 0613-2742AB) were carried out between 2003-2006 and 2009-2011 using the CTIO $1.5 \mathrm{~m}$ telescope under the aegis of the SMARTS Consortium. The CTIO $1.5 \mathrm{~m}$ Richey-Chretien Spectrograph (RCSpec) was used with the $32 /$ I grating setting, covering 6000-9600 $\AA$ at a resolution of $8 \AA$. Two spectra of each target were taken back-to-back to allow cosmic ray rejection. Data were reduced with standard IRAF techniques using one flux standard per night for absolute flux calibration. No telluric standards were observed, as the data were not originally intended for any purpose other than determining spectral types.

Spectral types were determined by direct comparison to previously obtained standard stars (Henry et al. 2002) spectra. The spectra were prepared by interpolating them onto a fixed $1 \AA$ grid running from 6000 to $9000 \AA$. We then removed telluric features (defined as any region with $>4 \%$ absorption from the Hinkle et al. 2003 sky absorption atlas) and $\mathrm{H} \alpha$. The target spectra were then compared to the standards by dividing target by standard after cropping both spectra to only the wavelengths where both spectra overlap. The lowest standard deviation stddev(target/standard) was taken as the best-matching spectral type. The resulting spectral types are presented in Column 14 of Table 2, and have \pm 0.5 type errors. Offsets compared to other spectral typing methods (Reid et al. 1995) are of similar size.

Spectral line EWs and indices were computed with the same program, utilizing $11 \AA$ windows centered on the maximum or minimum of the feature for both $\mathrm{H} \alpha$ and $\mathrm{K}_{\mathrm{I}} 7699 \AA$. Full bins of $24 \AA$ were used for the Na I doublet index. These features were measured prior to removing the telluric and $\mathrm{H} \alpha$ features.

\subsubsection{CTIO $4.0 \mathrm{~m}$ RCSpec}

We obtained spectra of SCR 0613-2742AB with the CTIO $4.0 \mathrm{~m}$ telescope's RCSpec on 2008 September 18 and 2008 September 19 using the KPGLF-1 $(632 \mathrm{~g} / \mathrm{mm})$ grating, which covers $4900-8050 \AA$ at a resolution of $1.9 \AA \mathrm{pixel}^{-1}$. The spectral type, $\mathrm{H} \alpha$, and $\mathrm{K}_{\mathrm{I}} \mathrm{EW}$ measurements of SCR 0613$2742 \mathrm{AB}$ come from spectra taken at this telescope, and were calibrated using the same program that analyzed the above CTIO $1.5 \mathrm{~m}$ RCSpec data.

\subsubsection{MPG $2.2 \mathrm{~m}$ FEROS}

One spectrum of SCR 0613-2742AB was taken with the FEROS spectrograph (Kaufer et al. 1999) on the MPG $2.2 \mathrm{~m}$ telescope at La Silla Observatory on 2013 February 18 as part of ESO program 090.C-0200(A). FEROS is an echelle spectrograph fed by two 2 ". 0 fibers and provides $R \sim 48,000$ spectra over a wavelength range of 3500-9200 A. Observations were taken in the object-sky mode with the use of the atmospheric dispersion corrector. The data were reduced with the facility pipeline and the IRAF task fxcor was used to cross-correlate the target spectrum with several radial velocity standards observed in the same fashion. We measure a heliocentric radial velocity of $+22.54 \pm 1.16 \mathrm{~km} \mathrm{~s}^{-1}$ for SCR 0613-2742AB (Table 4). The Na I gravity index measurement and Li $6708 \AA \mathrm{EW}$ for SCR 0613-2742AB were also derived from these spectra.

\subsubsection{CFHT ESPaDOnS}

SCR 1425-4113AB was observed on the Canada-FranceHawaii Telescope (CFHT) with the ESPaDOnS (Donati et al. 2006). ESPaDONs was used in the "star+sky" mode, to get a resolving power of $R=68,000$ covering 3700-10500 $\AA$ over 40 grating orders. The data were reduced by the queue service observing team using UPENA pipeline. We measure a heliocentric radial velocity, lithium $\mathrm{EW}$, and $v \sin i$ for both targets in the system.

\section{AN ASSESSMENT OF STELLAR YOUTH TRACERS}

Our available data-trigonometric parallaxes, low-resolution red optical spectroscopy, and VRIJHK photometry for the entire sample-provides four methods of young star identification at our disposal: $U V W X Y Z$ kinematics, isochrones, the Bayesian Analysis for Nearby Young AssociatioNs (BANYAN) statistical method, and gravity sensitive features. Taken individually, none offers conclusive proof of youth. Together, they present a strong case for the youth (and specific association membership) of the systems described here. The measured data used to make our conclusions are given in Tables 4-6; the results of our analysis are given in Table 7 .

\subsection{UVWXYZ Kinematics}

Stars that form together should be moving together through space. Over time, internal and external interactions will cause them to disperse into the thin disk of the Galaxy. The associations we consider here (Table 8) are all sufficiently young that this has not happened yet, even though (with the exception of $\eta$ Chameleontis, the Pleiades, and the Hyades) they are gravitationally unbound.

The perhaps hundreds of systems in an association are spread out over tens of thousands of cubic parsecs, interspersed among thousands of field systems (and, indeed, members of other young moving groups), and as an unbound association, their velocity dispersions are larger than $1 \mathrm{~km} \mathrm{~s}^{-1}$. Therefore, large numbers of older stars (López-Santiago et al. 2009 in particular quotes $\sim 30 \%$ ) will coincidentally happen to have matching $U V W$ motions, and even larger numbers of field stars will fall within the spatial boundaries of an association. $U V W$ motions do not prove youth, but they are necessary to connect young stars to young associations.

There is also the non-trivial issue of whether we have an accurate assemblage of these nearby associations and moving groups. We adopt the associations in Table 8 despite the knowledge that the physical reality of these groups (and the accuracy of their proposed members) is still somewhat uncertain. For instance, the IC 2391 Supercluster (Eggen 1991), Carina-Vela moving group (Makarov \& Urban 2000), and Argus association (Torres et al. 2008) have all been proposed as the extended halo of the nearby IC 2391 open cluster, but all are more or less distinct from each other in terms of membership and proposed properties; currently only Argus is thought to be an actual co-eval assembly. 
Table 4

Data Used to Calculate $U V W X Y Z$

\begin{tabular}{|c|c|c|c|c|c|c|c|c|c|}
\hline $\begin{array}{l}\text { Name } \\
\text { (1) }\end{array}$ & \multicolumn{2}{|c|}{$(\mathrm{J} 2000 \pm$ mas $)$} & $\begin{array}{c}\pi \\
\text { (mas) } \\
(4)\end{array}$ & $\begin{array}{c}\pi \\
\text { Ref. } \\
(5)\end{array}$ & \multicolumn{2}{|c|}{$\mu_{\left(\operatorname{mas}_{\text {R.A. }} \mathrm{yr}^{-1}\right)} \mu$} & $\begin{array}{c}\mu \\
\text { Ref. } \\
(8)\end{array}$ & $\begin{array}{c}\text { R.V. } \\
\left(\mathrm{km} \mathrm{s}^{-1}\right) \\
(9)\end{array}$ & $\begin{array}{l}\text { R.V } \\
\text { Ref } \\
(10)\end{array}$ \\
\hline NLTT 372 & $000851.788 \pm 70$ & $+204907.86 \pm 80$ & $11.66 \pm 1.44$ & 1 & $+120.7 \pm 12.1$ & $-76.0 \pm 9.4$ & 1 & $\cdots$ & \\
\hline SCR 0017-6645 & $001723.524 \pm 80$ & $-664512.46 \pm 110$ & $25.61 \pm 1.73$ & 1 & $+102.9 \pm 1.0$ & $-15.0 \pm 1.0$ & 2 & $+11.4 \pm 0.8$ & \\
\hline GJ 2006A & $002750.242 \pm 70$ & $-323306.17 \pm 70$ & $30.97 \pm 1.76$ & 11 & $+99.2 \pm 1.3$ & $-61.3 \pm 2.6$ & 2 & +8.4 & 1. \\
\hline GJ 2006B & $002750.362 \pm 70$ & $-323323.91 \pm 70$ & $30.97 \pm 1.76$ & 11 & $+117.2 \pm 4.1$ & $-31.5 \pm 5.8$ & 2 & $\cdots$ & \\
\hline GJ 2022A & $012427.693 \pm 60$ & $-335508.75 \pm 60$ & $39.50 \pm 1.28$ & 13 & $+163.3 \pm 12.1$ & $-125.0 \pm 9.5$ & 1 & $+18.4 \pm 1.0$ & \\
\hline GJ 2022C & $012427.692 \pm 60$ & $-335508.75 \pm 60$ & $39.50 \pm 1.28$ & 13 & $+154.8 \pm 12.1$ & $-119.8 \pm 9.5$ & 1 & $+19.4 \pm 2.7$ & \\
\hline GJ 2022B & $012430.621 \pm 60$ & $-335501.64 \pm 60$ & $39.50 \pm 1.28$ & 13 & $+157.5 \pm 12.1$ & $-125.1 \pm 9.4$ & 1 & $+18.3 \pm 0.5$ & \\
\hline LP 993-115 & $024510.711 \pm 70$ & $-434432.37 \pm 60$ & $87.37 \pm 1.33$ & 11 & $+22.7 \pm 12.1$ & $-387.2 \pm 9.4$ & 1 & $\ldots$ & \\
\hline LP 993-116AB & $024514.316 \pm 70$ & $-434410.60 \pm 60$ & $87.37 \pm 1.33$ & 11 & $+24.0 \pm 12.1$ & $-366.1 \pm 9.4$ & 1 & $\cdots$ & \\
\hline G 7-34 & $041718.521 \pm 60$ & $+084922.06 \pm 60$ & $73.27 \pm 1.27$ & 1 & $+121.6 \pm 12.1$ & $-373.8 \pm 9.4$ & 1 & $\cdots$ & \\
\hline G 39-29AB & $043812.592 \pm 470$ & $+281300.00 \pm 80$ & $75.03 \pm 1.89$ & 13 & $+386.9 \pm 12.2$ & $-90.7 \pm 9.5$ & 1 & $+35.7 \pm 1.2$ & \\
\hline L $449-1 \mathrm{AB}$ & $051722.908 \pm 60$ & $-352154.70 \pm 60$ & $84.38 \pm 1.35$ & 1 & $-234.2 \pm 12.1$ & $-162.6 \pm 9.5$ & 1 & $\cdots$ & \\
\hline SCR 0529-3239 & $052944.686 \pm 60$ & $-323914.17 \pm 60$ & $38.19 \pm 1.61$ & 1 & $+12.5 \pm 0.9$ & $+13.4 \pm 1.8$ & 2 & $\cdots$ & \\
\hline SCR 0613-2742AB & $061313.308 \pm 60$ & $-274205.46 \pm 60$ & $34.04 \pm 1.00$ & 1 & $-13.1 \pm 1.6$ & $-0.3 \pm 1.3$ & 2 & $+22.54 \pm 1.16$ & \\
\hline L $34-26$ & $074912.709 \pm 60$ & $-764206.60 \pm 60$ & $94.36 \pm 2.11$ & 1 & $-102.4 \pm 1.1$ & $-191.9 \pm 1.1$ & 2 & +0.9 & 10 \\
\hline SCR 0757-7114 & $075732.554 \pm 60$ & $-711453.81 \pm 70$ & $45.25 \pm 1.96$ & 1 & $+86.7 \pm 1.3$ & $+20.6 \pm 1.3$ & 2 & $\cdots$ & \\
\hline SCR 1012-3124AB & $101209.085 \pm 60$ & $-312445.20 \pm 60$ & $18.54 \pm 1.74$ & 1 & $-74.8 \pm 1.1$ & $-9.4 \pm 1.0$ & 2 & $+14.69 \pm 0.53$ & 10 \\
\hline TWA 8B & $113241.165 \pm 70$ & $-265209.04 \pm 70$ & $21.28 \pm 1.01$ & 11 & $-95.3 \pm 2.2$ & $-28.6 \pm 4.7$ & 2 & $+8.93 \pm 0.27$ & 17 \\
\hline TWA 8A & $113241.247 \pm 70$ & $-265155.94 \pm 70$ & $21.28 \pm 1.01$ & 11 & $-95.3 \pm 2.2$ & $-28.6 \pm 4.7$ & 2 & $+8.34 \pm 0.48$ & 17 \\
\hline SCR 1214-2345 & $121408.666 \pm 80$ & $-234517.06 \pm 70$ & $91.39 \pm 1.55$ & 1 & $+42.4 \pm 1.1$ & $+85.0 \pm 3.4$ & 2 & & \\
\hline G $165-8 \mathrm{AB}$ & $133146.620 \pm 70$ & $+291636.54 \pm 60$ & $55.51 \pm 2.38$ & 1 & $-244.1 \pm 4.2$ & $-132.4 \pm 4.8$ & 2 & $-7.5 \pm 6.5$ & 15 \\
\hline SCR $1425-4113 \mathrm{AB}$ & $142529.128 \pm 60$ & $-411332.40 \pm 60$ & $14.94 \pm 0.96$ & 1 & $-46.8 \pm 2.1$ & $-49.2 \pm 1.7$ & 2 & $-1.2 \pm 1.3$ & \\
\hline GJ 1224 & $180732.853 \pm 60$ & $-155747.05 \pm 60$ & $126.99 \pm 1.01$ & 14 & $-620.3 \pm 12.1$ & $-339.9 \pm 9.4$ & 1 & $-34.8 \pm 1.7$ & 15 \\
\hline G 141-29 & $184244.985 \pm 60$ & $+135417.05 \pm 70$ & $90.18 \pm 1.88$ & 14 & $-39.9 \pm 12.1$ & $+359.2 \pm 9.5$ & 1 & -33.2 & 15 \\
\hline LHS 3799 & $222306.997 \pm 60$ & $-173626.12 \pm 70$ & $137.69 \pm 1.75$ & 14 & $+285.9 \pm 12.1$ & $-711.3 \pm 9.4$ & 1 & $-2.1 \pm 1.1$ & 15 \\
\hline GJ 1284AB & $233013.447 \pm 60$ & $-202327.44 \pm 60$ & $65.79 \pm 1.86$ & 15 & $+305.3 \pm 12.1$ & $-187.5 \pm 9.5$ & 1 & -5.7 & 16 \\
\hline LHS 1302 & $015104.094 \pm 80$ & $-060705.07 \pm 80$ & $100.78 \pm 1.89$ & 6 & $+531.8 \pm 12.1$ & $-257.9 \pm 9.4$ & 6 & $\cdots$ & \\
\hline LHS 1358 & $021254.630 \pm 60$ & $+000016.81 \pm 70$ & $65.27 \pm 2.07$ & 7 & $+551.2 \pm 12.1$ & $+37.8 \pm 9.5$ & 7 & $\cdots$ & \\
\hline G 99-49 & $060003.515 \pm 60$ & $+024223.63 \pm 60$ & $190.77 \pm 1.86$ & 46 & $+305.2 \pm 12.1$ & $-41.7 \pm 9.5$ & 6 & $+28.7 \pm 0.7$ & 15 \\
\hline AP Col & $060452.157 \pm 60$ & $-343335.98 \pm 60$ & $119.21 \pm 0.98$ & 8 & $+21.4 \pm 12.1$ & $+341.2 \pm 9.4$ & 8 & $+22.4 \pm 0.3$ & 8 \\
\hline G 41-14ABC & $085856.332 \pm 60$ & $+082826.00 \pm 100$ & $147.66 \pm 1.98$ & 6 & $+371.2 \pm 3.5$ & $-323.4 \pm 2.8$ & 2 & $-6.4 \pm 19.0$ & 15 \\
\hline TWA 27AB & $120733.463 \pm 60$ & $-393254.01 \pm 60$ & $18.95 \pm 0.37$ & 91011 & $-68.5 \pm 12.2$ & $-22.5 \pm 9.5$ & 9 & $+7.5 \pm 2.0$ & 18 \\
\hline LHS 2729 & $132338.020 \pm 60$ & $-255445.12 \pm 70$ & $71.48 \pm 1.53$ & 7 & $-600.9 \pm 12.1$ & $-218.3 \pm 9.5$ & 7 & $\cdots$ & \\
\hline LHS 2836 & $135910.422 \pm 90$ & $-195003.62 \pm 60$ & $92.86 \pm 0.89$ & 7 & $-551.2 \pm 12.1$ & $-177.0 \pm 9.5$ & 7 & -15.8 & 15 \\
\hline GJ 1207 & $165705.729 \pm 60$ & $-042056.29 \pm 60$ & $115.26 \pm 1.50$ & 46 & $+479.1 \pm 12.1$ & $-367.1 \pm 9.5$ & 6 & $-4.2 \pm 1.6$ & 15 \\
\hline LHS 4016AB & $234836.062 \pm 60$ & $-273938.87 \pm 70$ & $41.25 \pm 1.55$ & 7 & $-551.9 \pm 12.1$ & $-239.8 \pm 9.5$ & 7 & $+25.3 \pm 0.55$ & 19 \\
\hline
\end{tabular}

Notes. References: 1: This paper, 2: UCAC4 (Zacharias et al. 2013), 3: Shkolnik et al. (2012), 4: YPC (van Altena et al. 1995), 5: Hipparcos (van Leeuwen 2007), 6: Henry et al. (2006), 7: Riedel et al. (2010), 8: Riedel et al. (2011), 9: Gizis et al. (2007) 10: Biller \& Close (2007), 11: Ducourant et al. (2008), 12: Malo et al. (2013), 13: L. Malo et al. (in preparation), 14: Montes et al. (2001), 15: Gizis et al. (2002), 16: Torres et al. (2006), 17: Shkolnik et al. (2011), 18: Rice et al. (2010), 19: Shkolnik et al. (2010). Parallaxes of multiple components in the same system have been combined, and are represented by duplicate references in the parallax column. All positions and position errors are taken from 2MASS (Skrutskie et al. 2006), and adjusted to epoch 2000.0 equinox J2000 using the proper motions listed here.

\subsubsection{The Kinematic Data}

Computing $U V W X Y Z$ kinematics requires R.A., decl., $\pi$, $\mu_{\text {R.A. cos decl., }} \mu_{\text {decl. }}$, and radial velocities for $U V W$ velocities; and R.A., Decl., and $\pi$ for $X Y Z$ positions. The input data used for this analysis (presented in Table 4) differs in sev- eral key ways from the pure CTIOPI astrometric data in Table 3.

For most star systems, the only available spectra are from the CTIO $1.5 \mathrm{~m}$ RCSpec, which lacks the resolution necessary for radial velocities. We have obtained radial velocities from the literature to fill out our sample. 
Table 5

Deblended Magnitudes for Isochrones

\begin{tabular}{|c|c|c|c|c|c|c|c|}
\hline $\begin{array}{l}\text { Name } \\
\text { (1) }\end{array}$ & $\begin{array}{c}V \\
(2)\end{array}$ & $\begin{array}{c}I \\
(3)\end{array}$ & $\begin{array}{c}J \\
(4)\end{array}$ & $\begin{array}{c}K \\
(5)\end{array}$ & $\begin{array}{r}M_{V} \\
(6)\end{array}$ & $\begin{array}{c}V-K \\
(7)\end{array}$ & $\begin{array}{c}\text { Deblend }^{\mathrm{a}} \\
(8)\end{array}$ \\
\hline NLTT 372 & 15.87 & 13.12 & 11.64 & 10.72 & 11.20 & 5.15 & \\
\hline G 131-26A & 13.94 & 10.98 & 9.40 & 8.81 & 12.61 & 5.13 & $K$ \\
\hline G 131-26B & 14.76 & 11.61 & 9.90 & 9.27 & 13.43 & 5.49 & $K$ \\
\hline SCR 0017-6645 & 12.45 & 10.00 & 8.56 & 7.70 & 9.49 & 4.75 & \\
\hline GJ 2006A & 12.95 & 10.29 & 8.88 & 8.01 & 10.40 & 4.94 & \\
\hline GJ 2006B & 13.25 & 10.48 & 8.97 & 8.12 & 10.70 & 5.13 & \\
\hline SCR 0103-5515A & 16.07 & 12.70 & 10.83 & 9.91 & 12.70 & 6.16 & $K$ \\
\hline SCR 0103-5515B & 16.43 & 12.97 & 11.04 & 10.11 & 13.06 & 6.32 & $K$ \\
\hline SCR 0103-5515C & 23.39 & 18.14 & 14.92 & 13.69 & 20.02 & 9.70 & $K$ \\
\hline LP 467-16A & 14.76 & 11.41 & 9.53 & 8.67 & 13.06 & 6.09 & $K$ \\
\hline LP 467-16B & 15.99 & 12.36 & 10.27 & 9.36 & 14.29 & 6.63 & $K$ \\
\hline GJ 2022A & 14.27 & 11.34 & 9.93 & 8.97 & 12.25 & 5.30 & $V$ \\
\hline GJ 2022C & 14.35 & 11.40 & 9.98 & 9.02 & 12.33 & 5.33 & $V$ \\
\hline GJ 2022B & 15.50 & 12.33 & 10.56 & 9.68 & 13.48 & 5.82 & \\
\hline LP 993-115 & 12.38 & 9.61 & 8.14 & 7.27 & 12.09 & 5.11 & \\
\hline LP 993-116A & 13.02 & 9.58 & 8.49 & 7.63 & 12.73 & 5.39 & $i^{\prime}$ \\
\hline LP 993-116B & 14.14 & 10.54 & 9.27 & 8.40 & 13.85 & 5.74 & $i^{\prime}$ \\
\hline G 7-34 & 13.84 & 10.75 & 9.03 & 8.18 & 13.16 & 5.66 & \\
\hline G 39-29A & 13.03 & 10.23 & 8.75 & 7.91 & 12.41 & 5.12 & $K$ \\
\hline G 39-29B & 13.69 & 10.74 & 9.14 & 8.28 & 13.07 & 5.41 & $K$ \\
\hline LP 655-48 & 17.79 & 13.36 & 10.66 & 9.55 & 17.85 & 8.24 & \\
\hline LP 476-207A & 12.46 & 9.75 & 8.30 & 7.48 & 10.48 & 4.98 & $\mathrm{SB}, K$ \\
\hline LP 476-207B & 13.56 & 10.41 & 8.65 & 7.76 & 11.58 & 5.80 & $K$ \\
\hline LP 476-207C & 12.46 & 9.75 & 8.30 & 7.48 & 10.48 & 4.98 & $\mathrm{SB}, K$ \\
\hline $\mathrm{BD}-21^{\circ} 1074 \mathrm{~B}$ & 11.44 & 8.86 & 7.47 & 6.60 & 10.02 & 4.84 & $R$ \\
\hline $\mathrm{BD}-21^{\circ} 1074 \mathrm{C}$ & 12.44 & 9.71 & 8.14 & 7.21 & 11.02 & 5.23 & $R$ \\
\hline $\mathrm{BD}-21^{\circ} 1074 \mathrm{~A}$ & 10.41 & 8.25 & 7.05 & 6.12 & 8.99 & 4.29 & \\
\hline L 449-1A & 12.05 & 9.32 & 7.87 & 7.05 & 11.68 & 5.00 & $R$ \\
\hline L 449-1B & 13.06 & 10.17 & 8.54 & 7.66 & 12.69 & 5.40 & $R$ \\
\hline SCR 0529-3239 & 13.79 & 10.80 & 9.22 & 8.32 & 11.70 & 5.47 & \\
\hline SCR 0613-2742A & 12.78 & 10.07 & 8.57 & 7.72 & 10.44 & 5.06 & $R$ \\
\hline SCR 0613-2742B & 13.41 & 10.60 & 8.98 & 8.11 & 11.07 & 5.30 & $R$ \\
\hline L 34-26 & 11.31 & 8.79 & 7.41 & 6.58 & 11.18 & 4.73 & \\
\hline SCR 0757-7114 & 12.45 & 9.77 & 8.32 & 7.42 & 10.73 & 5.03 & \\
\hline SCR 1012-3124A & 14.25 & 11.25 & 9.59 & 8.74 & 10.59 & 5.51 & $V$ \\
\hline SCR 1012-3124B & 14.28 & 11.27 & 9.61 & 8.75 & 10.62 & 5.53 & $V$ \\
\hline TWA 8B & 15.22 & 11.76 & 9.84 & 9.01 & 11.86 & 6.21 & \\
\hline TWA 8A & 12.23 & 9.79 & 8.34 & 7.43 & 8.87 & 4.80 & \\
\hline SCR 1214-2345 & 13.96 & 10.78 & 9.07 & 8.23 & 13.80 & 5.73 & \\
\hline G $165-8 A$ & 12.64 & 9.80 & 8.23 & 7.40 & 11.36 & 5.24 & $K$ \\
\hline G $165-8 B$ & 12.93 & 10.02 & 8.40 & 7.56 & 11.65 & 5.37 & $K$ \\
\hline SCR 1425-4113A & 13.29 & 10.81 & 9.30 & 8.37 & 9.16 & 4.92 & SB \\
\hline SCR 1425-4113B & 13.29 & 10.81 & 9.30 & 8.37 & 9.16 & 4.92 & SB \\
\hline GJ 1224 & 13.48 & 10.31 & 8.64 & 7.83 & 14.00 & 5.65 & \\
\hline G $141-29$ & 12.86 & 9.95 & 8.36 & 7.55 & 12.64 & 5.31 & \\
\hline SCR 1942-2045 & 14.33 & 11.25 & 9.60 & 8.76 & 13.31 & 5.57 & \\
\hline 2MASS 2009-0113 & 14.47 & 11.16 & 9.40 & 8.51 & 14.38 & 5.96 & \\
\hline SCR 2010-2801A & 13.62 & 10.85 & 9.32 & 8.41 & 10.22 & 5.21 & $R$ \\
\hline SCR 2010-2801B & 13.86 & 11.06 & 9.49 & 8.56 & 10.46 & 5.30 & $R$ \\
\hline LEHPM2-0783 & 17.17 & 13.03 & 10.66 & 9.72 & 16.13 & 7.46 & \\
\hline L 755-19 & 12.47 & 9.81 & 8.39 & 7.50 & 11.10 & 4.97 & \\
\hline SCR 2033-2556 & 14.87 & 11.57 & 9.71 & 8.88 & 11.45 & 5.99 & \\
\hline SCR 2036-3607 & 11.66 & 9.27 & 8.03 & 7.17 & 10.63 & 4.49 & \\
\hline GJ 799A & 11.09 & 8.14 & 6.55 & 5.69 & 11.12 & 5.40 & $V$ \\
\hline GJ 799B & 11.13 & 8.17 & 6.57 & 5.71 & 11.16 & 5.42 & $V$ \\
\hline LHS 3799 & 13.30 & 10.04 & 8.24 & 7.32 & 13.99 & 5.98 & \\
\hline GJ $1284 A$ & 11.89 & 9.34 & 7.95 & 7.08 & 10.98 & 4.81 & SB \\
\hline GJ 1284B & 11.89 & 9.34 & 7.95 & 7.08 & 10.98 & 4.81 & SB \\
\hline LHS 1302 & 14.49 & 11.16 & 9.41 & 8.55 & 14.51 & 5.94 & \\
\hline LHS 1358 & 13.58 & 10.66 & 9.06 & 8.17 & 12.65 & 5.41 & \\
\hline G 99-49 & 11.31 & 8.43 & 6.91 & 6.04 & 12.71 & 5.27 & \\
\hline AP Col & 12.96 & 9.60 & 7.74 & 6.87 & 13.34 & 6.09 & \\
\hline G 41-14A & 12.07 & 9.27 & 7.78 & 6.97 & 12.92 & 5.10 & $K, \mathrm{SB}$ \\
\hline G 41-14B & 12.07 & 9.27 & 7.78 & 6.97 & 12.92 & 5.10 & $K, \mathrm{SB}$ \\
\hline G 41-14C & 12.21 & 9.20 & 7.56 & 6.72 & 13.06 & 5.49 & $K$ \\
\hline
\end{tabular}

Table 5

(Continued)

\begin{tabular}{|c|c|c|c|c|c|c|c|}
\hline $\begin{array}{l}\text { Name } \\
\text { (1) }\end{array}$ & $\begin{array}{c}V \\
(2)\end{array}$ & $\begin{array}{c}I \\
(3)\end{array}$ & $\begin{array}{c}J \\
(4)\end{array}$ & $\begin{array}{c}K \\
(5)\end{array}$ & $\begin{array}{c}M_{V} \\
(6)\end{array}$ & $\begin{array}{c}V-K \\
(7)\end{array}$ & $\begin{array}{c}\text { Deblend }^{\mathrm{a}} \\
\text { (8) }\end{array}$ \\
\hline TWA 27A & 19.95 & 15.92 & 13.00 & 11.96 & 16.34 & 7.99 & $K$ \\
\hline TWA 27B & 28.91 & 22.74 & 18.33 & 16.94 & 25.30 & 11.97 & $K$ \\
\hline LHS 2729 & 12.89 & 10.14 & 8.66 & 7.78 & 12.16 & 5.11 & \\
\hline LHS 2836 & 12.88 & 9.90 & 8.33 & 7.45 & 12.72 & 5.44 & \\
\hline GJ 1207 & 12.25 & 9.43 & 7.97 & 7.12 & 12.56 & 5.13 & \\
\hline LHS 4016A & 13.09 & 10.65 & 9.34 & 8.50 & 11.17 & 4.59 & SB \\
\hline LHS 4016B & 13.09 & 10.65 & 9.34 & 8.50 & 11.17 & 4.59 & SB \\
\hline
\end{tabular}

Note. ${ }^{\text {a }}$ Band of known $\Delta$ mag used to deblend the photometry, or SB if system was identified only as a spectroscopic binary. No entry is given for single stars because deblending is not required.

We use weighted mean parallaxes for systems that have multiple reported parallaxes-either from multiple system components, or parallax determinations from other parallax programs. In doing so, we have made two key assumptions: that all components of a star system are at the same effective distance to currently achievable accuracy; and that all published parallaxes are reasonably free of systematics.

The CTIOPI pipeline produces relative proper motions (as shown in Table 3) by assuming each target field's astrometric reference stars have no net motion, which is not strictly true. In order to have unbiased results, we ideally want to use absolute proper motions for our kinematic determinations. Comparing our results to the absolute International Celestial Reference System (ICRS) proper motions ${ }^{18}$ from UCAC4 (Zacharias et al. 2013), we find mean offsets of $+5.12 \pm 12.80$ mas $\mathrm{yr}^{-1}$ in

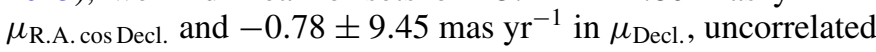
across the sky. The small differences but large uncertainties suggest CTIOPI proper motions are accurate relative to the UCAC4 ICRS grid, but the uncertainties are undersampled. Where possible, we use UCAC4 absolute proper motions directly. Where no UCAC4 proper motions are available, we use our proper motions, with the above offsets added in as a systematic uncertainty. In two cases-L 449-1AB and AP Col-the UCAC4 proper motion was discrepant with the CTIOPI proper motion by more than 100 mas $\mathrm{yr}^{-1}$, and we used the CTIOPI proper motion with the uncertainty.

\subsubsection{The Kinematic Method}

The standard method for computing $U V W$ space velocities is laid out in Johnson \& Soderblom (1987), and the matrices in that paper can easily be adapted to compute $X Y Z$ space positions. These $U V W X Y Z$ coordinates are right-handed Cartesian Galactic coordinates aligned so that the $U / X$ axis is toward the galactic center, the $V / Y$ axis is in the direction of galactic rotation, and the $W / Z$ axis is toward the north Galactic pole. In cases where we have full kinematic information, we calculate $10^{7}$ Monte Carlo iterations to fully sample the uncertainties on our input kinematics as a three-dimensional ellipsoid in velocity space. For stars without radial velocity measurements, we calculate $10^{5}$ Monte Carlo iterations at multiple different radial velocities within a range -100 to $+100 \mathrm{~km} \mathrm{~s}^{-1}$ (see Figure 2). $U V W X Y Z$ coordinates for the target objects are given in Table 9.

To determine whether a star system is a potential match for a given association, we must determine how close the

\footnotetext{
18 Note that UCAC4 also includes high proper motion objects from relative proper motion sources, including previous CTIOPI papers. Such entries are identifiable by flags within the database.
} 
Table 6

Photometric and Spectroscopic Properties

\begin{tabular}{|c|c|c|c|c|c|c|c|c|c|}
\hline $\begin{array}{l}\text { Name } \\
\text { (1) }\end{array}$ & $\begin{array}{c}M_{V} \\
(\mathrm{mag}) \\
(2)\end{array}$ & $\begin{array}{c}V-K_{S} \\
(\mathrm{mag}) \\
(3)\end{array}$ & $\begin{array}{c}\log \left(L_{X} / L_{\text {bol }}\right) \\
\text { (4) }\end{array}$ & $\begin{array}{c}\log L_{X} \\
\left(\text { erg }^{-1}\right) \\
(5)\end{array}$ & $\begin{array}{l}\text { Vari } \\
\text { Filter } \\
(6)\end{array}$ & $\begin{array}{l}\text { bility } \\
\text { (mag) } \\
\text { (7) }\end{array}$ & $\begin{array}{c}\mathrm{H} \alpha \\
\mathrm{EW} \mathrm{\AA} \\
(8)\end{array}$ & $\begin{array}{c}\mathrm{NaI} \\
\text { Index } \\
(9)\end{array}$ & $\begin{array}{c}\mathrm{K} \text { I } \\
\mathrm{EW} \AA \\
(10)\end{array}$ \\
\hline NLTT 372 & 11.20 & 5.15 & $(-2.22)$ & $(29.73)$ & $V$ & 0.014 & & & \\
\hline G 131-26AB & 12.19 & 5.51 & -3.28 & 28.40 & $V$ & 0.019 & -3.5 & 1.25 & 2.2 \\
\hline SCR 0017-6645 & 9.49 & 4.75 & -3.01 & 29.49 & $V$ & 0.035 & -4.8 & 1.11 & 0.5 \\
\hline GJ 2006A & 10.40 & 4.94 & -2.96 & 29.50 & $V$ & 0.077 & -5.6 & 1.13 & 0.6 \\
\hline GJ 2006B & 10.70 & 5.13 & & & $V$ & 0.036 & -10.0 & 1.15 & 1.1 \\
\hline SCR 0103-5515ABC & 12.11 & 6.24 & $(-4.57)$ & $(27.40)$ & $R$ & 0.021 & -14.5 & 1.25 & 3.0 \\
\hline LP 467-16AB & 12.76 & 6.25 & -3.08 & 28.62 & $R$ & 0.019 & -9.9 & 1.19 & 2.5 \\
\hline GJ 2022AC & 11.54 & 5.32 & -2.70 & 29.08 & $R$ & 0.067 & -9.4 & 1.23 & 2.1 \\
\hline GJ 2022B & 13.48 & 5.82 & $(-4.52)$ & $(26.87)$ & $R$ & 0.016 & -6.3 & 1.24 & 2.4 \\
\hline LP 993-115A & 12.09 & 5.11 & $(-5.43)$ & $(26.14)$ & $V$ & 0.021 & -1.6 & 1.19 & 1.5 \\
\hline LP 993-115BC & 12.40 & 5.49 & -3.24 & 28.35 & $V$ & 0.015 & -7.7 & 1.25 & 2.2 \\
\hline G 7-34 & 13.16 & 5.66 & -3.09 & 28.24 & $R$ & 0.022 & -8.4 & 1.19 & 2.0 \\
\hline G 39-29AB & 11.94 & 5.23 & -3.00 & 28.68 & $V$ & 0.015 & -4.7 & 1.19 & 2.1 \\
\hline LP $655-48$ & 17.85 & 8.24 & -2.74 & 27.63 & $I$ & 0.013 & -17.8 & 1.35 & 5.0 \\
\hline LP 476-207ABC & 9.55 & 5.16 & -3.11 & 29.50 & $V$ & 0.021 & -8.9 & 1.14 & 1.1 \\
\hline $\mathrm{BD}-21^{\circ} 1074 \mathrm{BC}$ & 9.66 & 4.97 & -3.24 & 29.58 & $V$ & 0.046 & -5.2 & 1.13 & 0.8 \\
\hline $\mathrm{BD}-21^{\circ} 1074 \mathrm{~A}$ & 8.99 & 4.29 & & & $V$ & 0.043 & -2.0 & 1.10 & 0.7 \\
\hline L 449-1AB & 11.32 & 5.13 & -3.02 & 28.88 & $V$ & 0.024 & -5.9 & 1.21 & 1.6 \\
\hline SCR 0529-3239 & 11.70 & 5.47 & -3.09 & 28.77 & $R$ & 0.014 & -6.1 & 1.18 & 1.5 \\
\hline SCR 0613-2742AB & 9.96 & 5.16 & -2.95 & 29.50 & $V$ & 0.037 & -6.0 & 1.10 & 0.8 \\
\hline L $34-26$ & 11.18 & 4.73 & -3.07 & 28.76 & $V$ & 0.018 & -4.9 & 1.18 & 1.0 \\
\hline SCR 0757-7114 & 10.73 & 5.03 & $(-5.55)$ & $(26.54)$ & $V$ & 0.007 & 0.3 & 1.17 & 0.7 \\
\hline SCR 1012-3124AB & 9.85 & 5.52 & $(-4.84)$ & $(27.76)$ & $V$ & 0.015 & -5.7 & 1.08 & 0.6 \\
\hline TWA 8B & 11.86 & 6.21 & -2.99 & 29.86 & $V$ & 0.124 & -13.8 & 1.14 & 1.5 \\
\hline TWA 8A & 8.87 & 4.80 & & & $V$ & 0.078 & -9.2 & 1.12 & 0.5 \\
\hline SCR 1214-2345 & 13.76 & 5.73 & -3.00 & 28.12 & $V$ & 0.010 & -7.4 & 1.35 & 3.6 \\
\hline G $165-8 \mathrm{AB}$ & 10.74 & 5.30 & -3.16 & 29.02 & $R$ & 0.018 & -7.3 & 1.17 & 1.3 \\
\hline SCR 1425-4113AB & 8.41 & 4.92 & $(-5.02)$ & $(27.97)$ & $V$ & 0.078 & -7.0 & 1.08 & 0.6 \\
\hline GJ 1224 & 14.00 & 5.65 & -3.03 & 27.97 & $I$ & 0.013 & -4.3 & 1.26 & 3.1 \\
\hline G 141-29 & 12.64 & 5.31 & -3.27 & 28.16 & $I$ & 0.013 & -4.7 & 1.22 & 2.0 \\
\hline SCR 1942-2045 & 13.32 & 5.57 & -2.89 & 28.37 & $R$ & 0.019 & -5.4 & 1.24 & 2.5 \\
\hline 2MASS 2009-0113 & 14.38 & 5.96 & -3.31 & 27.64 & $I$ & 0.015 & -5.5 & 1.28 & 3.3 \\
\hline SCR 2010-2801AB & 9.58 & 5.25 & -3.03 & 29.60 & $R$ & 0.012 & -9.8 & 1.15 & 1.1 \\
\hline LEHPM2-0783 & 16.13 & 7.46 & -2.20 & 28.57 & $I$ & 0.025 & -26.6 & 1.34 & 5.6 \\
\hline L 755-19 & 11.10 & 4.97 & -3.25 & 28.69 & $R$ & 0.018 & -5.0 & 1.17 & 1.1 \\
\hline SCR 2033-2556 & 11.45 & 5.99 & -3.10 & 29.03 & $R$ & 0.017 & -12.6 & 1.18 & 2.0 \\
\hline SCR 2036-3607 & 10.63 & 4.49 & -2.94 & 29.02 & $V$ & 0.021 & -3.2 & 1.13 & 0.9 \\
\hline GJ 799AB & 10.39 & 4.67 & -2.85 & 29.52 & $V$ & 0.064 & -10.8 & 1.16 & 1.5 \\
\hline LHS 3799 & 13.99 & 5.98 & -3.20 & 27.90 & $V$ & 0.014 & -3.8 & 1.31 & 2.9 \\
\hline GJ 1284AB & 10.23 & 4.81 & -3.23 & 29.00 & $V$ & 0.027 & -3.7 & 1.17 & 1.0 \\
\hline LHS 1302 & 14.51 & 5.94 & -3.32 & 27.58 & $R$ & 0.021 & -3.6 & 1.27 & 3.1 \\
\hline LHS 1358 & 12.85 & 5.41 & -3.23 & 28.22 & $R$ & 0.015 & & & \\
\hline G 99-49 & 12.71 & 5.27 & -3.40 & 27.98 & $V$ & 0.017 & -4.6 & 1.22 & 2.1 \\
\hline AP Col & 13.34 & 6.09 & -2.92 & 28.49 & $V$ & 0.017 & -18.4 & 1.17 & 2.2 \\
\hline G 41-14ABC & 11.77 & 5.23 & -2.94 & 28.81 & $V$ & 0.013 & -4.0 & 1.22 & 2.0 \\
\hline TWA 27A & 16.34 & 8.00 & $(-3.30)$ & $(27.58)$ & $I$ & 0.015 & & & \\
\hline LHS 2729 & 12.16 & 5.11 & -3.21 & 28.33 & $R$ & 0.012 & -3.4 & 1.16 & 1.7 \\
\hline LHS 2836 & 12.72 & 5.44 & -3.22 & 28.25 & $V$ & 0.013 & -3.7 & 1.22 & 2.0 \\
\hline GJ 1207 & 12.56 & 5.13 & -3.11 & 28.30 & $V$ & 0.199 & -4.9 & 1.17 & 1.3 \\
\hline LHS 4016AB & 10.42 & 4.60 & -3.14 & 28.94 & $V$ & 0.014 & -2.1 & 1.14 & 1.0 \\
\hline
\end{tabular}

Notes. Errors on X-ray values are less than $25 \%$. Errors on spectroscopic EW measures are roughly $0.2 \AA$. Errors on the $\mathrm{Na}$ I index are 0.05 . Values in parentheses are upper limits. Three systems have multiple resolved components within the RASS positional uncertainties, and have combined X-ray luminosities.

three-dimensional velocity-space ellipsoid(s) defined by our Monte Carlo iterations is to the three-dimensional velocity-space ellipsoid of the association, as defined in Table 8. Because the dispersions are meaningful-in the case of the sample system, they represent the uncertainty on the velocity of the system; in the case of the association, they represent the intrinsic dispersion of real members-our phase-space "separations" are calculated relative to those dispersions, in the form of our goodness-of-fit statistic $\gamma$ :

$$
\begin{aligned}
\gamma= & \frac{1}{3}\left(\frac{\left(U_{\text {assoc }}-U_{\text {system }}\right)^{2}}{\left(\sigma_{U_{\text {assoc }}}^{2}+\sigma_{U_{\text {system }}}^{2}\right)}+\frac{\left(V_{\text {assoc }}-V_{\text {system }}\right)^{2}}{\left(\sigma_{V_{\text {assoc }}}^{2}+\sigma_{V_{\text {system }}}^{2}\right)}\right. \\
& \left.+\frac{\left(W_{\text {assoc }}-W_{\text {system }}\right)^{2}}{\left(\sigma_{W_{\text {assoc }}}^{2}+\sigma_{W_{\text {system }}}^{2}\right)}\right) .
\end{aligned}
$$


Table 7

Young Star Results

\begin{tabular}{|c|c|c|c|c|c|c|c|c|c|c|}
\hline \multirow{2}{*}{$\begin{array}{l}\text { Name } \\
\text { (1) }\end{array}$} & \multicolumn{3}{|c|}{ Kinematic } & \multicolumn{2}{|c|}{ BANYAN } & \multirow{2}{*}{$\begin{array}{l}\text { Isochrone } \\
\text { Range } \\
\text { (7) }\end{array}$} & \multirow{2}{*}{$\begin{array}{c}\text { Gravity } \\
\text { Match } \\
\text { (8) }\end{array}$} & \multirow{2}{*}{$\begin{array}{c}\mathrm{H} \alpha \text { Age } \\
(\mathrm{Gyr}) \\
(9)\end{array}$} & \multirow{2}{*}{$\begin{array}{c}\text { Association } \\
\text { (10) }\end{array}$} & \multirow{2}{*}{$\begin{array}{c}\text { Other } \\
\text { References } \\
\text { (11) }\end{array}$} \\
\hline & $\begin{array}{l}\text { Match } \\
\text { (2) }\end{array}$ & $\begin{array}{l}\gamma \\
(3)\end{array}$ & $\begin{array}{l}\text { R.V. } \\
\text { (4) }\end{array}$ & $\begin{array}{l}\text { Match } \\
\text { (5) }\end{array}$ & $\begin{array}{c}\text { Prob (\%) } \\
\text { (6) }\end{array}$ & & & & & \\
\hline NLTT 372 & & & & Field & 99.9 & $\mathrm{ABD}-\beta \mathrm{Pic}$ & $\mathrm{N} / \mathrm{A}$ & $\mathrm{N} / \mathrm{A}$ & & \\
\hline G $131-26 \mathrm{AB}$ & & & & Field & 99.9 & Field-ABD & Old & $<4.5$ & & \\
\hline SCR 0017-6645 & $\beta$ Pic & 0.24 & +11.0 & $\beta$ Pic & 99.9 & $\beta$ Pic & Young & $<1.6$ & $\beta$ Pictoris & Riedel (2012), Malo et al. (2013) \\
\hline GJ $2006 \mathrm{AB}$ & $\beta$ Pic & 1.86 & $+11.4^{\mathrm{a}}$ & $\beta$ Pic & 99.9 & $\beta$ Pic & Young & $<3.0$ & $\beta$ Pictoris & Riedel (2012) \\
\hline SCR 0103-5515ABC & Carina & 1.68 & $+14.3^{\mathrm{a}}$ & Tuc-Hor & 98.9 & TWA- $\beta$ Pic & Young & $<6.0$ & Unknown & (Delorme et al. 2012 Tucana-Horologium) \\
\hline LP 467-16AB & $\beta$ Pic & 0.46 & +4.0 & $\beta$ Pic & 99.0 & $\beta$ Pic-Field & Young & $<6.0$ & $\beta$ Pictoris & Riedel (2012), Malo et al. (2013) \\
\hline GJ 2022ABC & AB Dor & 0.09 & +18.4 & AB Dor & 99.9 & Field & Young & $<4.5$ & AB Doradus & Riedel (2012), Shkolnik et al. (2012) \\
\hline LP 993-115ABC & & & & Field & 99.9 & Field & Old & $<3.0$ & & \\
\hline G 7-34 & AB Dor & 2.82 & $+18.0^{\mathrm{a}}$ & AB Dor & 99.9 & Field & Young & $<4.5$ & AB Doradus & Riedel (2012) \\
\hline G 39-29AB & & & & Field & 99.9 & Field & Old & $<4.5$ & & \\
\hline LP 655-48 & & & & Field & 99.9 & Field & Old & $<7.5$ & & \\
\hline LP 476-207ABC & $\beta$ Pic & 0.64 & +14.7 & $\beta$ Pic & 99.9 & $\beta$ Pic & Young & $<2.0$ & $\beta$ Pictoris & Song et al. (2003) \\
\hline $\mathrm{BD}-21^{\circ} 1074 \mathrm{ABC}$ & $\beta \mathrm{Pic}$ & 0.72 & +21.2 & $\beta$ Pic & 99.9 & $\beta$ Pic & Young & $<1.0$ & $\beta$ Pictoris & Torres et al. (2008) \\
\hline L $449-1 \mathrm{AB}$ & Ursa Major & 1.69 & $-2.3^{\mathrm{a}}$ & Field & 99.9 & Field & Old & $<3.0$ & Ursa Major? & Riedel (2012) \\
\hline SCR 0529-3239 & $\beta$ Pic & 2.74 & $+24.8^{\mathrm{a}}$ & $\beta$ Pic & 99.9 & $\beta$ Pic-Field & Young & $<4.5$ & $\beta$ Pictoris & Riedel (2012) \\
\hline SCR 0613-2742AB & $\beta$ Pic & 0.44 & +22.5 & $\beta$ Pic & 99.9 & $\beta$ Pic & Young & $<4.5$ & $\beta$ Pictoris & Riedel (2012), Malo et al. (2013) \\
\hline L 34-26 & Ursa Major & 3.87 & $+6.7^{\mathrm{a}}$ & Field & 99.9 & Field & Old & $<2.0$ & & \\
\hline SCR 0757-7114 & & & & Field & 99.9 & $\beta$ Pic-ABD & Young? & $>3.0$ & & \\
\hline SCR 1012-3124AB & TW Hya & 3.20 & +14.7 & TW Hya & 99.9 & TWA $-\beta$ Pic & Young & $<4.5$ & TW Hydra & Riedel (2012) \\
\hline TWA 8AB & TW Hya & 3.26 & +8.3 & TW Hya & 99.9 & TWA & Young & $<4.5$ & TW Hydra & Webb et al. (1999) \\
\hline SCR $1214-2345$ & & & & Field & 99.9 & Field & Old & $<4.5$ & & \\
\hline G $165-8 \mathrm{AB}$ & Carina & 0.06 & -7.5 & Columba & 74.7 & $\beta$ Pic-Field & Young & $<4.0$ & Unknown & Riedel (2012) \\
\hline SCR $1425-4113 \mathrm{AB}$ & $\beta$ Pic & 0.59 & -3.7 & TW Hya & 75.0 & $>$ TWA & Young & $<1.6$ & TW Hydra? & Riedel (2012) \\
\hline GJ 1224 & & & & Field & 99.9 & Field & Old & $<4.5$ & & \\
\hline G $141-29$ & Hyades & 3.68 & $-38.2^{\mathrm{a}}$ & Field & 99.9 & Field & Old & $<4.5$ & & \\
\hline SCR 1942-2045 & & & & Field & 99.9 & Field & Old & $<4.5$ & & \\
\hline 2MASS 2009-0113 & & & & Field & 99.9 & Field & Old & $<6.0$ & & \\
\hline SCR $2010-2801 \mathrm{AB}$ & $\beta$ Pic & 2.05 & $-10.0^{\mathrm{a}}$ & $\beta$ Pic & 99.9 & TWA $-\beta$ Pic & Young & $<4.5$ & $\beta$ Pictoris & Riedel (2012), Malo et al. (2013) \\
\hline LEHPM2-0783 & & & & Field & 99.9 & TWA-Field & Old & $<7.5$ & & \\
\hline L $755-19$ & Argus & 3.21 & -25.4 & Argus & 99.9 & $\beta$ Pic-Field & Old & $<2.0$ & Argus? & Riedel (2012) \\
\hline SCR 2033-2556 & $\beta \mathrm{Pic}$ & 0.75 & $-9.1^{\mathrm{a}}$ & $\beta$ Pic & 99.9 & TWA & Young & $<6.0$ & $\beta$ Pictoris & Riedel (2012), Malo et al. (2013) \\
\hline SCR 2036-3607 & Ursa Major & 1.22 & $+20.9^{\mathrm{a}}$ & Field & 99.9 & Field & Young & $<1.6$ & & \\
\hline GJ 799AB & $\beta \mathrm{Pic}$ & 0.93 & -3.7 & $\beta$ Pic & 99.9 & $\beta$ Pic & Young & $<4.5$ & $\beta$ Pictoris & Barrado y Navascués et al. (1999) \\
\hline LHS 3799 & & & & Field & 94.1 & Field & Old & $<4.5$ & & \\
\hline GJ $1284 \mathrm{AB}$ & Columba & 0.27 & $-3.7^{\mathrm{a}}$ & $\beta$ Pic & 51.9 & $\beta$ Pic-Field & Old & $<2.0$ & & \\
\hline LHS 1302 & Columba & 2.89 & $+10.2^{\mathrm{a}}$ & Field & 87.6 & Field & Old & $<6.0$ & & \\
\hline LHS 1358 & Hyades & 1.13 & $+22.9^{\mathrm{a}}$ & Field & 99.9 & Field & $\mathrm{N} / \mathrm{A}$ & $\mathrm{N} / \mathrm{A}$ & & \\
\hline G 99-49 & & & & Field & 99.9 & Field & Old & $<3.0$ & & (Montes et al. 2001, Hyades) \\
\hline AP Col & Argus & 0.18 & +22.4 & Argus & 99.9 & $\beta$ Pic-Field & Young & $<6.0$ & Argus & Riedel et al. (2011) \\
\hline G 41-14ABC & Ursa Major & 0.51 & -6.4 & Field & 99.9 & Field & Old & $<3.0$ & & \\
\hline TWA $27 \mathrm{AB}$ & TW Hya & 0.23 & +7.5 & TW Hya & 99.9 & TW Hya & $\mathrm{N} / \mathrm{A}$ & $\mathrm{N} / \mathrm{A}$ & TW Hydra & Gizis et al. (2002) \\
\hline LHS 2729 & & & & Field & 99.9 & Field & Old & $<3.0$ & & \\
\hline LHS 2836 & & & & Field & 99.9 & Field & Old & $<4.5$ & & \\
\hline GJ 1207 & & & & Field & 99.9 & Field & Old & $<3.0$ & & \\
\hline LHS 4016AB & & & & Field & 99.9 & Field & Old & $<1.6$ & & \\
\hline
\end{tabular}

Note. ${ }^{a}$ R.V. is actually a best-fit R.V. from the kinematic fitting.

This $\gamma$ statistic is effectively identical to the one used in Shkolnik et al. (2012), where it appears as $\bar{\chi}^{2}$.

We take a value of $\gamma$ less than 4 as a potentially significant match. In many cases, a system will be kinematically consistent with membership in more than one young association. This is often unavoidable, as the velocity distributions of several associations genuinely overlap; in these cases we must look at the other diagnostics to determine which association is the most consistent with the available data. In cases where we have no radial velocity, we fit the resulting $\gamma$ values from our range of points to determine a best-fit radial velocity and $\gamma$. Only the $\gamma$ value for the most consistent association is given in Column 5 of Table 7 .

\subsection{Isochrones}

Pre-main-sequence stars are still in the process of contracting under gravity, and are still physically larger than main-sequence stars of the same temperature, and consequently much brighter. It is therefore possible to distinguish stars of a given age using isochrones, or at least demonstrate the youth of a system.

\subsubsection{The Photometric Data}

Overluminosity is also a sign of multiplicity, and must therefore be taken into account before making conclusions about the potential youth of a system based on its luminosity. Our sample has many multiple stars (see Table 10), which are not 

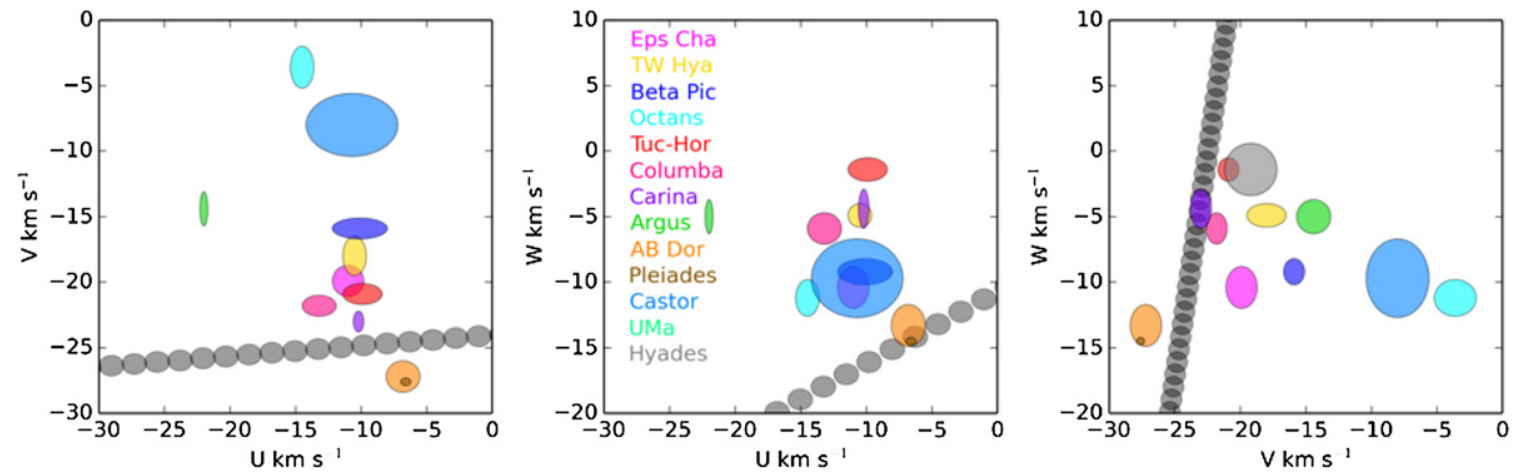

Figure 2. Kinematic $U V W$ diagrams for G 7-34. Because we have no radial velocity for G 7-34, $U V W$ velocities (gray) have been calculated for a range of input radial velocities, producing the "string of pearls" effect. The only possible agreement is with the AB Doradus association, with a best-fit radial velocity of $+18.0 \mathrm{~km} \mathrm{~s}{ }^{-1}$ (which is suggestive, but not necessarily correct). A similar analyses were carried out for all stars without known radial velocities.

(A color version of this figure is available in the online journal.)

Table 8

Nearby Young Associations

\begin{tabular}{|c|c|c|c|c|c|c|c|c|c|c|c|c|c|}
\hline Name & $\begin{array}{c}U \\
\left(\mathrm{~km} \mathrm{~s}^{-1}\right) \\
(2)\end{array}$ & $\begin{array}{c}\sigma_{U} \\
\left(\mathrm{~km} \mathrm{~s}^{-1}\right) \\
(3)\end{array}$ & $\begin{array}{c}V \\
\left(\mathrm{~km} \mathrm{~s}^{-1}\right) \\
(4)\end{array}$ & $\begin{array}{c}\sigma_{V} \\
\left(\mathrm{~km} \mathrm{~s}^{-1}\right) \\
(5)\end{array}$ & $\begin{array}{c}W \\
\left(\mathrm{~km} \mathrm{~s}^{-1}\right) \\
(6)\end{array}$ & $\begin{array}{c}\sigma_{W} \\
\left(\mathrm{~km} \mathrm{~s}^{-1}\right) \\
(7)\end{array}$ & $\begin{array}{c}\min X \\
(\mathrm{pc}) \\
(8)\end{array}$ & $\begin{array}{c}\max X \\
(\mathrm{pc}) \\
(9)\end{array}$ & $\begin{array}{c}\min Y \\
(\mathrm{pc}) \\
(10)\end{array}$ & $\begin{array}{c}\max Y \\
(\mathrm{pc}) \\
(11)\end{array}$ & $\begin{array}{c}\min Z \\
(\mathrm{pc}) \\
(12)\end{array}$ & $\begin{array}{c}\max Z \\
(\mathrm{pc}) \\
(13)\end{array}$ & $\begin{array}{c}\text { Age } \\
(\mathrm{Myr}) \\
(14)\end{array}$ \\
\hline$\epsilon$ Cha & -11.0 & 1.2 & -19.9 & 1.2 & -10.4 & 1.6 & +34 & +60 & -105 & -78 & -44 & -12 & $6^{\mathrm{a}}$ \\
\hline TW Hya & -10.5 & 0.9 & -18.0 & 1.5 & -4.9 & 0.9 & +2 & +34 & -61 & -26 & +10 & +27 & $8^{a}$ \\
\hline$\beta$ Pic & -10.1 & 2.1 & -15.9 & 0.8 & -9.2 & 1.0 & -32 & +76 & -33 & 21 & -29 & -1 & $12^{\mathrm{a}}$ \\
\hline Octans & -14.5 & 0.9 & -3.6 & 1.6 & -11.2 & 1.4 & -79 & +142 & -138 & -60 & -85 & -38 & 20 \\
\hline Tuc-Hor & -9.9 & 1.5 & -20.9 & 0.8 & -1.4 & 0.9 & -61 & +43 & -47 & -4 & -44 & -30 & 30 \\
\hline Columba & -13.2 & 1.3 & -21.8 & 0.8 & -5.9 & 1.2 & -106 & +9 & -168 & +1 & -99 & +6 & 30 \\
\hline Carina & -10.2 & 0.4 & -23.0 & 0.8 & -4.4 & 1.5 & -2 & +33 & -154 & -39 & -33 & +5 & 30 \\
\hline Argus & -22.0 & 0.3 & -14.4 & 1.3 & -5.0 & 1.3 & -55 & +64 & -154 & -6 & -67 & +8 & 50 \\
\hline AB Dor & -6.8 & 1.3 & -27.2 & 1.2 & -13.3 & 1.6 & -94 & +73 & -131 & +58 & -66 & +23 & $125^{\mathrm{b}}$ \\
\hline Pleiades $^{\mathrm{c}}$ & -6.6 & 0.4 & -27.6 & 0.3 & -14.5 & 0.3 & -134 & -108 & +14 & +40 & -66 & -40 & $125^{\mathrm{b}}$ \\
\hline Castor $^{\mathrm{d}}$ & -10.7 & 3.5 & -8.0 & 2.4 & -9.7 & 3.0 & $\ldots$ & $\ldots$ & $\ldots$ & $\ldots$ & $\ldots$ & $\ldots$ & 200 \\
\hline $\mathrm{UMa}^{\mathrm{e}}$ & +14.56 & 2.28 & +2.81 & 1.75 & -8.37 & 3.42 & $\ldots$ & $\ldots$ & $\ldots$ & $\ldots$ & $\ldots$ & $\ldots$ & 500 \\
\hline Hyades $^{\mathrm{f}}$ & -41.1 & 2.0 & -19.2 & 2.0 & -1.4 & 2.0 & -53 & -33 & -9.3 & +10.7 & -27.3 & +7.3 & 650 \\
\hline
\end{tabular}

Notes. All data (unless otherwise specified) from Torres et al. (2008).

${ }^{a}$ Only these ages are known with any degree of certainty or corroboration.

${ }^{\mathrm{b}}$ Age from Luhman et al. (2005).

${ }^{c}$ Dimensions Soderblom et al. (2005), 13 pc tidal radius Adams et al. (2001).

d Barrado y Navascues (1998).

e King et al. (2003).

${ }^{\mathrm{f}}$ Röser et al. (2011).

resolved in CTIOPI images if they are less than $\sim 2^{\prime \prime}$ apart. In order to properly distinguish between binaries (which in the maximal case of an equal-luminosity pair can be $0.7 \mathrm{mag}$ brighter than a single star) and young stars, we have made an extensive literature search for multiples within the sample. Once multiples are identified, their photometry must be deblended to properly place them on a color-magnitude diagram.

Unfortunately, we do not have both $\Delta V$ and $\Delta K_{S}$ values for any multiples in our sample, so the deblending in one of the filters must be estimated. Plotting, for instance, $M_{V}$ versus $M_{K_{S}}$ (Figure 3) demonstrates that the relation is, to a first approximation, linear between $M_{V}=1$ and $M_{V}=15$ $\left(M_{K_{S}}=1\right.$ and $\left.M_{K_{S}}=9\right)$, in that case with a slope of 1.8 , for $\Delta V=1.8 \times \Delta K_{S}$. We list all the equivalencies in Table 11 . Actual measurements should definitely be preferred, however, as the error on the fit slopes are on the order of 0.03 , and the residuals to the fits are on the order of 0.5 .

For purposes of deblending our stars, we have assumed that the $\Delta \mathrm{FGS} 583 \mathrm{~W}$ values are equivalent to Kron-Cousins $\Delta R$, and that all the various $\Delta K$ values (CIT, Altair, MKO, 2MASS) are equivalent to 2MASS $\Delta K_{S}$. Several systems are known only as spectroscopic binaries. With no other information available, we have assumed $\Delta V=\Delta K_{S}=0$. The photometric data used in the isochrone analysis, including deblended magnitudes, are given in Table 5 and used to place stars on the color-magnitude diagram in Figure 4. Deblended optical magnitudes are given for the brown dwarfs TWA 27B and SCR 0103-5515C, but their deblended $V$ magnitudes are suspect at best.

\subsubsection{The Photometric Method}

Because isochrones do not match field main-sequence stars well at low masses (Hillenbrand \& White 2004), we are using empirical "isochrones" from Riedel et al. (2011), which are empirical polynomial fits derived from known young stars with photometry and trigonometric parallaxes, for the four associations-TW Hya, $\beta$ Pic, Tuc-Hor, and AB Dor-with a sufficient number of known low-mass members to make the isochrones useful. Plotting our stars on color-magnitude 
Table 9

UVWXYZ Kinematics

\begin{tabular}{|c|c|c|c|c|c|c|}
\hline Name & $\begin{array}{c}U \\
\left(\mathrm{~km} \mathrm{~s}^{-1}\right) \\
(2)\end{array}$ & $\begin{array}{c}V \\
\left(\mathrm{~km} \mathrm{~s}^{-1}\right) \\
(3)\end{array}$ & $\begin{array}{c}W \\
\left(\mathrm{~km} \mathrm{~s}^{-1}\right) \\
(4)\end{array}$ & $\begin{array}{c}X \\
(\mathrm{pc}) \\
(5)\end{array}$ & $\begin{array}{c}Y \\
(\mathrm{pc}) \\
(6)\end{array}$ & $\begin{array}{c}Z \\
(\mathrm{pc}) \\
(7)\end{array}$ \\
\hline NLTT 372 & $\cdots$ & $\cdots$ & $\cdots$ & $-21.86 \pm 2.73$ & $+61.00 \pm 7.61$ & $-56.25 \pm 7.01$ \\
\hline G 131-26AB & $\cdots$ & $\cdots$ & $\cdots$ & $-4.71 \pm 0.12$ & $+13.13 \pm 0.33$ & $-12.10 \pm 0.30$ \\
\hline SCR 0017-6645 & $-11.30 \pm 1.12$ & $-16.71 \pm 0.87$ & $-9.19 \pm 0.63$ & $+15.49 \pm 1.05$ & $-19.72 \pm 1.33$ & $-29.92 \pm 2.02$ \\
\hline GJ 2006A & $\cdots$ & $\cdots$ & $\cdots$ & $+4.04 \pm 0.23$ & $-1.10 \pm 0.06$ & $-32.01 \pm 1.80$ \\
\hline GJ 2006B & $\cdots$ & $\cdots$ & $\cdots$ & $+4.05 \pm 0.23$ & $-1.10 \pm 0.06$ & $-32.02 \pm 1.82$ \\
\hline SCR 0103-5515ABC & $\cdots$ & $\cdots$ & $\cdots$ & $+10.93 \pm 0.70$ & $-19.50 \pm 1.26$ & $-41.64 \pm 2.68$ \\
\hline LP $467-16 \mathrm{AB}$ & $-12.34 \pm 1.16$ & $-16.58 \pm 1.19$ & $-9.87 \pm 0.72$ & $-9.55 \pm 0.37$ & $+11.37 \pm 0.44$ & $-16.01 \pm 0.62$ \\
\hline GJ 2022A & $-7.71 \pm 1.36$ & $-26.52 \pm 1.47$ & $-13.93 \pm 1.02$ & $-0.85 \pm 0.03$ & $-4.24 \pm 0.14$ & $-24.95 \pm 0.81$ \\
\hline GJ 2022C & $-6.72 \pm 1.36$ & $-25.58 \pm 1.51$ & $-15.12 \pm 2.67$ & $-0.85 \pm 0.03$ & $-4.24 \pm 0.14$ & $-24.95 \pm 0.81$ \\
\hline GJ 2022B & $-6.55 \pm 1.36$ & $-26.10 \pm 1.45$ & $-13.92 \pm 0.56$ & $-0.86 \pm 0.03$ & $-4.24 \pm 0.14$ & $-24.95 \pm 0.81$ \\
\hline LP 993-115 & $\cdots$ & $\cdots$ & $\cdots$ & $-1.28 \pm 0.02$ & $-5.27 \pm 0.08$ & $-10.08 \pm 0.16$ \\
\hline LP 993-116AB & $\cdots$ & $\cdots$ & $\cdots$ & $-1.28 \pm 0.02$ & $-5.27 \pm 0.08$ & $-10.08 \pm 0.15$ \\
\hline G 7-34 & $\cdots$ & $\cdots$ & $\cdots$ & $-11.95 \pm 0.21$ & $-0.96 \pm 0.02$ & $-6.52 \pm 0.11$ \\
\hline G 39-29A & $-40.54 \pm 1.19$ & $-14.62 \pm 0.84$ & $+6.88 \pm 0.82$ & $-12.89 \pm 0.33$ & $+1.84 \pm 0.05$ & $-2.87 \pm 0.07$ \\
\hline G 39-29B & $-32.71 \pm 1.57$ & $-15.74 \pm 0.86$ & $+8.62 \pm 0.85$ & $-12.89 \pm 0.33$ & $+1.84 \pm 0.05$ & $-2.87 \pm 0.07$ \\
\hline LP 655-48 & $-30.67 \pm 0.33$ & $-14.73 \pm 0.47$ & $-1.84 \pm 0.47$ & $-7.69 \pm 0.05$ & $-3.12 \pm 0.02$ & $-5.09 \pm 0.03$ \\
\hline LP 476-207ABC & $-10.12 \pm 3.47$ & $-13.55 \pm 1.50$ & $-9.10 \pm 1.77$ & $-23.15 \pm 1.19$ & $-4.30 \pm 0.22$ & $-8.06 \pm 0.42$ \\
\hline $\mathrm{BD}-21^{\circ} 1074 \mathrm{BC}$ & $-12.92 \pm 1.07$ & $-17.06 \pm 0.99$ & $-11.06 \pm 0.93$ & $-12.01 \pm 0.30$ & $-11.00 \pm 0.28$ & $-10.25 \pm 0.26$ \\
\hline $\mathrm{BD}-21^{\circ} 1074 \mathrm{~A}$ & $-12.37 \pm 0.57$ & $-16.07 \pm 0.54$ & $-8.06 \pm 0.50$ & $-12.01 \pm 0.30$ & $-11.00 \pm 0.28$ & $-10.24 \pm 0.26$ \\
\hline L $449-1 \mathrm{AB}$ & $\cdots$ & $\cdots$ & $\cdots$ & $-5.07 \pm 0.08$ & $-8.47 \pm 0.14$ & $-6.56 \pm 0.11$ \\
\hline SCR 0529-3239 & $\cdots$ & $\cdots$ & $\cdots$ & $-12.41 \pm 0.52$ & $-18.83 \pm 0.79$ & $-13.28 \pm 0.56$ \\
\hline SCR 0613-2742AB & $-12.43 \pm 0.65$ & $-16.42 \pm 0.90$ & $-9.34 \pm 0.45$ & $-15.99 \pm 0.47$ & $-22.49 \pm 0.66$ & $-10.07 \pm 0.30$ \\
\hline L $34-26$ & $\cdots$ & $\cdots$ & $\cdots$ & $+3.16 \pm 0.07$ & $-9.22 \pm 0.21$ & $-4.16 \pm 0.09$ \\
\hline SCR 0757-7114 & $\cdots$ & $\cdots$ & $\cdots$ & $+4.91 \pm 0.21$ & $-20.11 \pm 0.87$ & $-7.74 \pm 0.34$ \\
\hline SCR 1012-3124A & $-14.59 \pm 1.34$ & $-17.80 \pm 0.63$ & $-7.61 \pm 1.23$ & $-2.38 \pm 0.22$ & $-50.55 \pm 4.75$ & $+18.65 \pm 1.75$ \\
\hline SCR 1012-3124B & $-14.57 \pm 1.34$ & $-17.55 \pm 0.80$ & $-7.70 \pm 1.24$ & $-2.38 \pm 0.22$ & $-50.55 \pm 4.75$ & $+18.65 \pm 1.75$ \\
\hline TWA 8B & $-14.10 \pm 0.98$ & $-18.15 \pm 0.74$ & $-6.54 \pm 1.01$ & $+8.21 \pm 0.39$ & $-38.65 \pm 1.84$ & $+25.44 \pm 1.21$ \\
\hline TWA 8A & $-14.20 \pm 0.39$ & $-17.67 \pm 1.83$ & $-6.85 \pm 1.03$ & $+8.21 \pm 0.39$ & $-38.65 \pm 1.83$ & $+25.44 \pm 1.21$ \\
\hline SCR 1214-2345 & $\cdots$ & $\cdots$ & $\cdots$ & $+3.22 \pm 0.06$ & $-7.96 \pm 0.14$ & $+6.79 \pm 0.12$ \\
\hline G $165-8 \mathrm{AB}$ & $-10.17 \pm 0.87$ & $-22.38 \pm 1.26$ & $-3.81 \pm 6.42$ & $+1.88 \pm 0.08$ & $+2.16 \pm 0.09$ & $+17.79 \pm 0.76$ \\
\hline SCR $1425-4113 \mathrm{AB}$ & $-10.95 \pm 1.24$ & $-16.35 \pm 1.43$ & $-8.85 \pm 0.86$ & $+49.64 \pm 3.19$ & $-39.70 \pm 2.55$ & $+20.97 \pm 1.35$ \\
\hline GJ 1224 & $-28.98 \pm 1.65$ & $-30.09 \pm 0.57$ & $+12.77 \pm 0.45$ & $+7.64 \pm 0.06$ & $+1.87 \pm 0.02$ & $+0.30 \pm 0.01$ \\
\hline G 141-29 & $\cdots$ & $\cdots$ & $\cdots$ & $+7.84 \pm 0.16$ & $+7.68 \pm 0.16$ & $+1.58 \pm 0.03$ \\
\hline SCR 1942-2045 & $+1.08 \pm 0.20$ & $-11.11 \pm 0.25$ & $-2.28 \pm 0.20$ & $+14.14 \pm 0.20$ & $+4.94 \pm 0.07$ & $-5.50 \pm 0.08$ \\
\hline 2MASS 2009-0113 & $\cdots$ & $\cdots$ & $\cdots$ & $+7.50 \pm 0.13$ & $+6.51 \pm 0.11$ & $-3.20 \pm 0.05$ \\
\hline SCR 2010-2801AB & $\cdots$ & $\cdots$ & $\cdots$ & $+40.83 \pm 2.63$ & $+10.28 \pm 0.66$ & $-22.93 \pm 1.48$ \\
\hline LEHPM2-0783 & $\cdots$ & $\cdots$ & $\cdots$ & $+12.46 \pm 0.21$ & $-4.76 \pm 0.08$ & $-9.11 \pm 0.15$ \\
\hline L 755-19 & $\cdots$ & $\cdots$ & $\cdots$ & $+14.02 \pm 0.44$ & $+9.24 \pm 0.29$ & $-8.46 \pm 0.27$ \\
\hline SCR 2033-2556 & $\cdots$ & $\cdots$ & $\cdots$ & $+38.49 \pm 2.66$ & $+12.69 \pm 0.88$ & $-26.32 \pm 1.82$ \\
\hline SCR 2036-3607 & $\cdots$ & $\cdots$ & $\cdots$ & $+12.96 \pm 0.29$ & $+1.45 \pm 0.03$ & $-9.43 \pm 0.21$ \\
\hline GJ 799A & $-7.99 \pm 2.40$ & $-17.47 \pm 0.65$ & $-9.01 \pm 1.84$ & $+7.80 \pm 0.06$ & $+1.54 \pm 0.01$ & $-5.85 \pm 0.04$ \\
\hline GJ 799B & $-9.30 \pm 2.40$ & $-13.86 \pm 0.65$ & $-11.49 \pm 1.84$ & $+7.80 \pm 0.06$ & $+1.54 \pm 0.01$ & $-5.85 \pm 0.04$ \\
\hline LHS 3799 & $-0.02 \pm 0.61$ & $-24.92 \pm 0.60$ & $-8.95 \pm 0.94$ & $+3.20 \pm 0.04$ & $+2.74 \pm 0.04$ & $-5.92 \pm 0.08$ \\
\hline GJ $1284 \mathrm{AB}$ & $\cdots$ & $\cdots$ & $\cdots$ & $+3.40 \pm 0.10$ & $+3.85 \pm 0.11$ & $-14.31 \pm 0.40$ \\
\hline LHS 1302 & $\ldots$ & $\cdots$ & $\cdots$ & $-3.98 \pm 0.08$ & $+1.48 \pm 0.03$ & $-8.97 \pm 0.17$ \\
\hline LHS 1358 & $\cdots$ & $\cdots$ & $\cdots$ & $-8.04 \pm 0.25$ & $+2.60 \pm 0.08$ & $-12.78 \pm 0.40$ \\
\hline G 99-49 & $-24.82 \pm 0.64$ & $-16.29 \pm 0.37$ & $+1.05 \pm 0.31$ & $-4.69 \pm 0.05$ & $-2.15 \pm 0.02$ & $-0.92 \pm 0.01$ \\
\hline AP Col & $-22.00 \pm 0.37$ & $-13.46 \pm 0.36$ & $-4.65 \pm 0.45$ & $-3.72 \pm 0.03$ & $-6.70 \pm 0.06$ & $-3.41 \pm 0.03$ \\
\hline G 41-14ABC & $+16.03 \pm 12.28$ & $-5.62 \pm 10.37$ & $+1.62 \pm 10.10$ & $-4.38 \pm 0.06$ & $-3.70 \pm 0.05$ & $+3.60 \pm 0.05$ \\
\hline TWA 27A & $-10.33 \pm 2.88$ & $-15.76 \pm 2.30$ & $-5.14 \pm 2.35$ & $+19.66 \pm 0.38$ & $-44.60 \pm 0.87$ & $+20.23 \pm 0.40$ \\
\hline LHS 2729 & $\ldots$ & $\ldots$ & $\ldots$ & $+7.53 \pm 0.16$ & $-8.38 \pm 0.18$ & $+8.30 \pm 0.18$ \\
\hline LHS 2836 & $\cdots$ & $\cdots$ & $\cdots$ & $+6.65 \pm 0.06$ & $-4.83 \pm 0.05$ & $+6.96 \pm 0.07$ \\
\hline GJ 1207 & $+6.19 \pm 1.44$ & $-0.83 \pm 0.57$ & $-24.39 \pm 0.82$ & $+7.72 \pm 0.10$ & $+2.05 \pm 0.03$ & $+3.38 \pm 0.04$ \\
\hline LHS 4016AB & $+72.89 \pm 2.85$ & $+4.57 \pm 1.15$ & $-9.44 \pm 0.85$ & $+5.20 \pm 0.20$ & $+2.66 \pm 0.10$ & $-23.53 \pm 0.88$ \\
\hline
\end{tabular}

Notes. Six-dimensional phase space positions for the stars in this sample.

diagrams with these isochrones (Figure 4) demonstrates that many are indeed overluminous relative to main-sequence stars. Note that several stars (SCR 0103-5515C, LP 655-48, TWA $27 \mathrm{~A}$ and B) are too red and faint to appear in the figures, and the Tuc-Hor isochrone line from Riedel et al. (2011) does not extend into the $\mathrm{M}$ dwarfs shown due to lack of data.

\subsection{BANYAN}

BANYAN (Malo et al. 2013) is an independent Bayesian methodology for finding young stars. BANYAN uses $I J$ photometry, Baraffe et al. (1998, 2002) model isochrones, and a slightly different set of $U V W X Y Z$ values for the known 
Table 10

Multiple Star Parameters

\begin{tabular}{|c|c|c|c|c|c|c|c|c|c|c|}
\hline $\begin{array}{l}\text { Primary } \\
\text { Name } \\
\text { (1) }\end{array}$ & $\begin{array}{l}\text { Secondary } \\
\text { Name } \\
(2)\end{array}$ & $\begin{array}{c}\text { Separation } \\
\text { (arcsec) } \\
(3)\end{array}$ & $\begin{array}{c}\text { Position Angle } \\
\left({ }^{\circ}\right) \\
(4)\end{array}$ & $\begin{array}{c}\text { Ref. } \\
\text { (5) }\end{array}$ & $\begin{array}{c}\Delta \mathrm{mag} \\
(6)\end{array}$ & $\begin{array}{l}\text { Filter } \\
\text { (7) }\end{array}$ & $\begin{array}{l}\text { Ref. } \\
(8)\end{array}$ & $\begin{array}{l}\text { Period } \\
\text { (9) }\end{array}$ & $\begin{array}{l}\text { Ref. } \\
\text { (10) }\end{array}$ & $\begin{array}{l}\text { Resolving } \\
\text { Obs. } \\
(11)\end{array}$ \\
\hline G 131-26 A & G 131-26 B & 0.111 & 169.9 & 2 & 0.46 & $\mathrm{MKO} K_{s}$ & 2 & $\sim 4 \mathrm{y}$ & 2 & $\mathrm{AO}$ \\
\hline GJ $2006 \mathrm{~A}$ & GJ 2006 B & 17.9 & 4.9 & 1 & 0.46 & Johnson $V$ & 1 & $\cdots$ & $\cdots$ & VB \\
\hline SCR 0103-5515 A & SCR 0103-5515 B & 0.249 & 61.0 & $3^{\mathrm{a}}$ & 0.20 & $2 \mathrm{MASS} K_{s}$ & 3 & $\ldots$ & $\ldots$ & $\mathrm{AO}$ \\
\hline SCR 0103-5515 AB & SCR 0103-5515 C & 1.77 & 336.1 & 3 & 4.43 & $2 \mathrm{MASS} K_{s}$ & 3 & $\cdots$ & $\cdots$ & $\mathrm{AO}$ \\
\hline LP 467-16 A & LP 467-16 B & 0.409 & 147.2 & 2 & 0.69 & MKO $K_{s}$ & 2 & $\cdots$ & $\cdots$ & $\mathrm{AO}$ \\
\hline GJ $2022 \mathrm{AC}$ & GJ 2022 B & 37.2 & 78.8 & $1^{\mathrm{a}}$ & 1.94 & Johnson $V$ & $1^{\mathrm{a}}$ & $\cdots$ & $\cdots$ & VB \\
\hline GJ $2022 \mathrm{~A}$ & GJ 2022 C & 1.8 & 224.4 & 1 & 0.08 & Johnson $V$ & 1 & $\cdots$ & $\cdots$ & VB \\
\hline LP 993-115 & LP 993-116 AB & 44.8 & 60.9 & $1^{\mathrm{a}}$ & 0.31 & Johnson $V$ & $1^{\mathrm{a}}$ & $\ldots$ & $\ldots$ & VB \\
\hline LP 993-116 A & LP 993-116 B & 0.257 & 214.6 & 4 & 0.87 & SDSS $i^{\prime}$ & 4 & $\cdots$ & $\cdots$ & LI, PB \\
\hline G 39-29 A & G 39-29 B & 0.87 & 300.6 & 5 & 0.37 & Altair $K_{s}$ & 5 & $\cdots$ & $\ldots$ & $\mathrm{AO}$ \\
\hline LP 476-207 AC & LP 476-207 B & 1.0 & 165 & 6 & 1.03 & CIT $K$ & 6 & $\cdots$ & $\cdots$ & SP \\
\hline LP 476-207 A & LP 476-207 C & $\cdots$ & $\cdots$ & $\cdots$ & $\cdots$ & $\cdots$ & $\cdots$ & $12 \mathrm{~d}$. & 7 & SB \\
\hline $\mathrm{BD}-21^{\circ} 1074 \mathrm{~A}$ & $\mathrm{BD}-21^{\circ} 1074 \mathrm{BC}$ & 8.3 & 229.3 & $1^{\mathrm{a}}$ & 0.67 & Johnson $V$ & $1^{\mathrm{a}}$ & $\cdots$ & $\cdots$ & VB \\
\hline $\mathrm{BD}-21^{\circ} 1074 \mathrm{~B}$ & $\mathrm{BD}-21^{\circ} 1074 \mathrm{C}$ & 0.760 & 148.2 & 1 & 0.94 & FGS F583W & 1 & $\cdots$ & $\cdots$ & VB, FGS, PB \\
\hline L 449-1 A & L 449-1 B & 0.047 & 332.8 & 1 & 0.95 & FGS F583W & 1 & $\sim 2.5 \mathrm{y}$ & 1 & FGS, PB \\
\hline SCR 0613-2742 A & SCR 0613-2742 B & 0.093 & $\ldots \mathrm{b}$ & 1 & 0.59 & FGS F583W & 1 & $>4 \mathrm{y}$ & 1 & FGS, PB, SB \\
\hline G 41-14 AB & G 41-14 C & 0.62 & $\ldots$ & $7^{\mathrm{a}}$ & 0.5 & MKO $K$ & 7 & $\sim 10 \mathrm{y}$ & 7 & $\mathrm{AO}$ \\
\hline G 41-14 A & G 41-14 B & $\cdots$ & $\cdots$ & $\cdots$ & $\cdots$ & $\cdots$ & $\cdots$ & $7.6 \mathrm{~d}$. & 7 & SB \\
\hline SCR 1012-3124 A & SCR 1012-3124 B & 1 & 270 & $1^{\mathrm{c}}$ & 0.03 & Johnson $V$ & $1^{\mathrm{c}}$ & $\cdots$ & $\cdots$ & $\mathrm{VB}, \mathrm{SB}$ \\
\hline TWA 8A & TWA 8B & 13.1 & 184.7 & 1 & 2.97 & Johnson $V$ & 1 & $\cdots$ & $\cdots$ & VB \\
\hline TWA 27A & TWA 27B & 0.778 & 125.8 & 8 & 4.98 & 2 MASS $K_{s}$ & 8 & $\cdots$ & $\cdots$ & $\mathrm{AO}$ \\
\hline G $165-8$ A & G $165-8$ B & 0.17 & 253.3 & 2 & 0.16 & $\operatorname{MKO} K_{s}$ & 2 & $\ldots$ & $\cdots$ & $\mathrm{AO}$ \\
\hline SCR 1425-4113 A & SCR 1425-4113 B & $\cdots$ & $\cdots$ & $\cdots$ & $\cdots$ & $\cdots$ & $\cdots$ & $\cdots$ & 1 & SB \\
\hline SCR 2010-2801 A & SCR 2010-2801 B & 0.614 & 281.6 & 1 & 0.23 & FGS F583W & 1 & $\cdots$ & $\cdots$ & FGS \\
\hline GJ 803 & GJ $799 \mathrm{AB}$ & 4681.0 & 212.4 & $1^{\mathrm{a}}$ & 1.71 & Johnson $V$ & $1^{\mathrm{a}}$ & $\cdots$ & $\ldots$ & VB \\
\hline GJ 799 A & GJ 799 B & 2.3 & 156 & 1 & 0.04 & Johnson $V$ & 1 & $\cdots$ & $\cdots$ & $\mathrm{VB}, \mathrm{PB}$ \\
\hline GJ $1284 \mathrm{~A}$ & GJ $1284 \mathrm{~B}$ & $\cdots$ & $\cdots$ & $\cdots$ & $\cdots$ & $\cdots$ & $\cdots$ & $\cdots$ & 9 & SB \\
\hline LHS $4016 \mathrm{~A}$ & LHS 4016 B & $\cdots$ & $\cdots$ & $\cdots$ & $\ldots$ & $\ldots$ & $\ldots$ & $<20 \mathrm{~d}$ & 10 & SB \\
\hline
\end{tabular}

Notes. VB = Visual Binary. $\mathrm{SB}=$ Spectroscopic Binary. $\mathrm{PB}=$ Astrometric Binary with perturbation in CTIOPI data. AO $=$ Resolved by AO. LI $=$ Resolved by Lucky Imaging. SP = Resolved by Speckle Interferometry. FGS = Resolved by FGS Interferometry. References: (1) This work; (2) Beuzit et al. (2004); (3) Delorme et al. (2013); (4) Bergfors et al. (2010); (5) Daemgen et al. (2007); (6) Henry et al. (1997); (7) Delfosse et al. (1999); (8) Chauvin et al. (2004); (9) Torres et al. (2006); (10) Shkolnik et al. (2010).

a Relative to unresolved components.

b Ambiguous sign on $X$-axis of solution.

${ }^{c}$ Estimate from PSF peaks.

associations. It searches for members of the known nearby associations $\beta$ Pictoris, TW Hydra, Tucana-Horologium, Columba, Carina, Argus, and AB Doradus, with "Field" as the default hypothesis. It is presented as a complementary, proven method. The coefficients for the best-matching association are shown in Table 7, Columns 5 and 6.

\subsection{Low Surface Gravity}

Three gravity-sensitive features exist within the $6000 \AA$ $9000 \AA$ coverage of our spectra: Ca II $(8498,8542,8662 \AA)$ is strong in giants and weak in dwarfs; Na I $(8183,8195 \AA)$ and K I $(7665,7699 \AA)$ are weak in giants and strong in dwarfs. The general pattern is that the neutral alkali and alkali earth metals are increasingly strong with higher gravity, while their singly ionized variants grow weaker with higher gravity (Allers et al. 2007; Schlieder et al. 2012a).
In this paper we are using the $\mathrm{Na}$ I index as defined in Lyo et al. (2004a), and the K I $7699 \AA$ doublet line (the other is contaminated by the atmospheric $A$ band). The $\mathrm{Na}$ I index value is formed by the ratio of the average flux in two $24 \AA$ wide bands:

$$
\mathrm{NaI}_{\text {index }}=\frac{F_{8148-8172}}{F_{8176-8200}} .
$$

Measurements for the program stars are given in Table 6, and graphs of the general trends are shown in Figure 5. Unfortunately, as can be seen in the figures, young stars and main-sequence stars overlap at colors bluer than $V-K_{s}=5$, in both cases. The lines can also be affected by stellar activity, where emission fills in the absorption line cores, leading to lower EWs (Reid \& Hawley 1999).

In principle, multiplicity and metallicity will have an effect on these features. The effects are muted in the case of multiplicity, 
Table 11

Deblending Ratios

\begin{tabular}{lccccccccc}
\hline \hline Desired & \multicolumn{7}{c}{ Observed } \\
\cline { 2 - 10 } & $\Delta V_{\mathrm{J}}$ & $\Delta R_{\mathrm{KC}}$ & $\Delta I_{\mathrm{KC}}$ & $\Delta g^{\prime}{ }_{\text {APASS }}$ & $\Delta r^{\prime}{ }_{\text {APASS }}$ & $\Delta i^{\prime}{ }_{\text {APASS }}$ & $\Delta J_{2 \text { MASS }}$ & $\Delta H_{2 \mathrm{MASS}}$ & $\Delta K_{S 2 \mathrm{MASS}}$ \\
\hline$\Delta V_{\mathrm{J}}$ & 1.00 & 1.06 & 1.36 & 1.03 & 1.07 & 1.28 & 1.67 & 1.73 & 1.80 \\
$\Delta R_{\mathrm{KC}}$ & 0.94 & 1.00 & 1.11 & 1.19 & 1.03 & 1.12 & 1.41 & 1.46 & 1.53 \\
$\Delta I_{\mathrm{KC}}$ & 0.74 & 0.90 & 1.00 & 0.97 & 1.03 & 1.10 & 1.26 & 1.31 & 1.37 \\
$\Delta g^{\prime}{ }_{\text {APASS }}$ & 0.97 & 0.84 & 1.03 & 1.00 & 1.04 & 1.24 & 1.38 & 1.38 & 1.43 \\
$\Delta r^{\prime}{ }_{\text {APASS }}$ & 0.93 & 0.97 & 0.97 & 0.96 & 1.00 & 1.18 & 1.32 & 1.31 & 1.36 \\
$\Delta i^{\prime}{ }_{\text {APASS }}$ & 0.78 & 0.89 & 0.91 & 0.81 & 0.85 & 1.00 & 1.11 & 1.10 & 1.14 \\
$\Delta J_{2 \text { MASS }}$ & 0.60 & 0.71 & 0.79 & 0.72 & 0.76 & 0.90 & 1.00 & 1.03 & 1.07 \\
$\Delta H_{2 \text { MASS }}$ & 0.58 & 0.68 & 0.76 & 0.72 & 0.76 & 0.91 & 0.97 & 1.00 & 1.04 \\
$\Delta K_{\text {S2MASS }}$ & 0.56 & 0.65 & 0.73 & 0.70 & 0.74 & 0.88 & 0.93 & 0.96 & 1.00 \\
\hline
\end{tabular}

Notes. Deblending ratios, in the sense of $\Delta Y=n \times \Delta X\left(\Delta V_{\mathrm{J}}=1.80 \Delta K_{s 2 \mathrm{MASS}}\right)$. Errors on the ratios themselves are \pm 0.03 ; residuals of the fits are 0.5. $V_{\mathrm{J}}$ data were taken from Bessell $(1990,1991)$, Weis $(1993,1996)$; Weis et al. (1999), Koen et al. (2002), and previous papers in this series. $R_{\mathrm{KC}} I_{\mathrm{KC}}$ were taken from only the Bessell papers. $g^{\prime} r^{\prime} i^{\prime}$ were taken from APASS DR6. $J H K_{s}$ were taken from the 2MASS Point Source Catalog (Skrutskie et al. 2006). Parallax data were taken from the RECONS 25 pc Database, which is being prepared for publication.

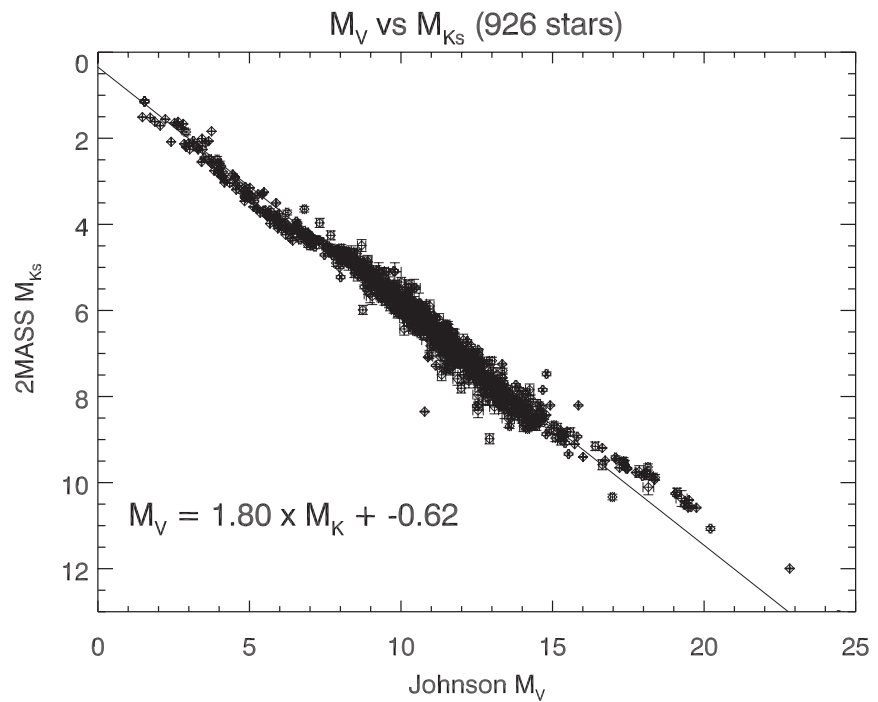

Figure 3. Approximately linear relationship between Johnson $M_{V}$ and 2MASS $M_{K_{S}}$, as determined from 962 stars with trigonometric parallax errors less than 10 mas. The values here can be used to convert $\Delta V$ measurements into $\Delta K_{s}$ and vice versa. $V$ data from Bessell $(1990,1991)$, and previous papers in this series; $K_{S}$ data from 2MASS (Skrutskie et al. 2006). Other relations are given in Table 11.

as the brighter component will dominate, and the line will not be appreciably weaker or stronger than that of a mainsequence star of the same color. Metallicity is more difficult. While we can expect low-metallicity subdwarfs to have weaker lines due to lower abundances, subdwarfs plot below the main sequence and will not be mistaken for pre-main-sequence stars. However, as noted by Shkolnik et al. (2009), highmetallicity stars of a given mass and bolometric luminosity will masquerade as stars of lower temperature and lower surface gravity. The additional metals increase the opacity of the stellar atmosphere and therefore put the effective photosphere farther from the center of the star. Thus, these stars will appear above the main sequence-in fact, these gravity-sensitive atomic species are the ones used by Rojas-Ayala et al. (2010) to measure the metallicity of field $\mathrm{M}$ dwarfs.

With the exception of SCR 0613-2742AB and SCR 1425$4113 \mathrm{AB}$, the only available spectra for the sample stars are low-resolution flux-calibrated optical spectra from the CTIO
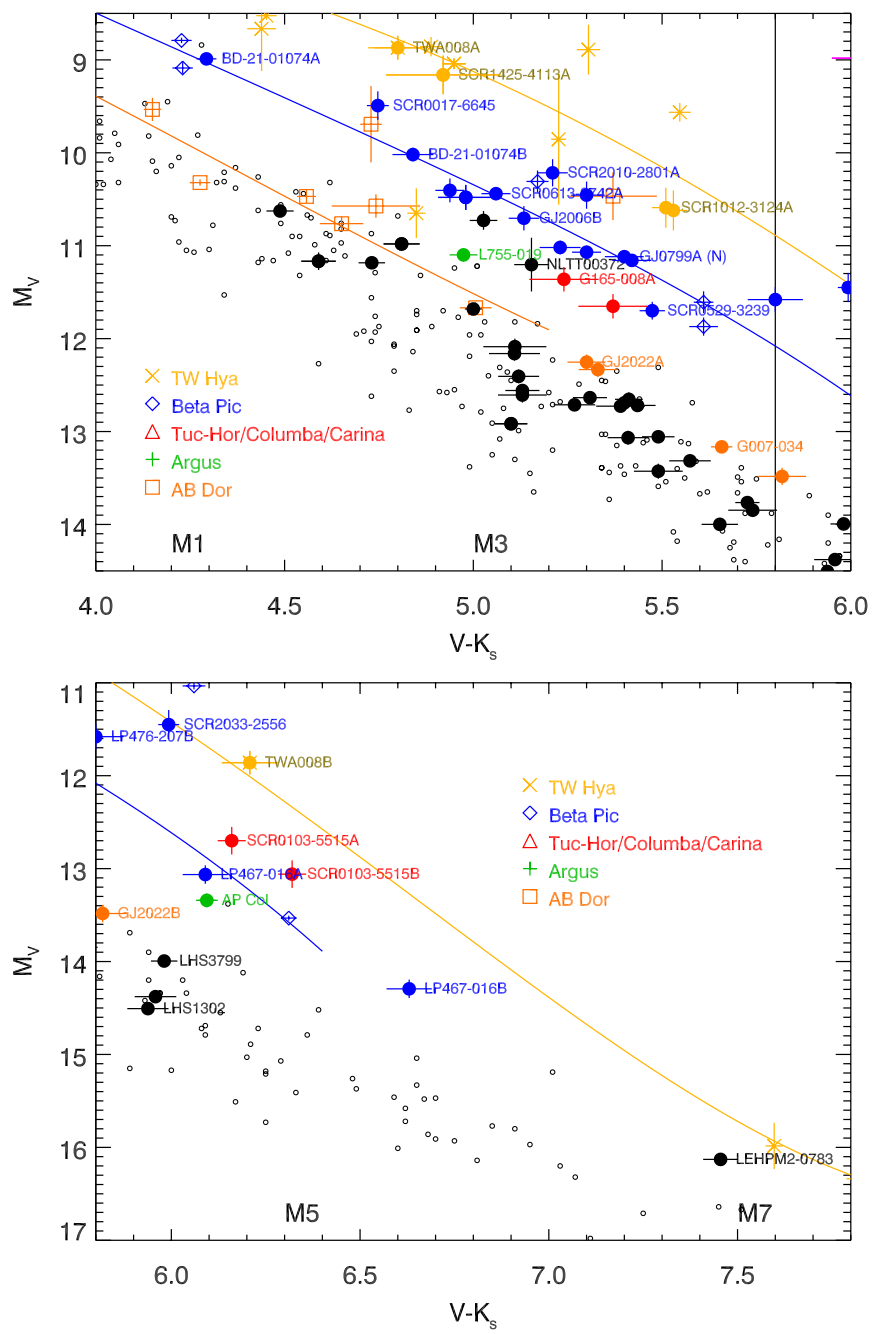

Figure 4. Known components of the 45 systems described in this paper (excepting the extremely red objects TWA 27AB, LP 655-48, and SCR 0103-5515C), deblended where necessary and plotted relative to the RECONS $10 \mathrm{pc}$ sample (open circles) on two overlapping color-magnitude diagrams. Also plotted are members of nearby young associations: TW Hya (Xs), $\beta$ Pic (diamonds), TucHor (triangles), and $\mathrm{AB}$ Dor (squares). Fifth order fits are plotted for (top to bottom) TW Hya, $\beta$ Pic, and AB Dor. The line at $V-K_{S}=5.8$ is roughly the point at which $\mathrm{M}$ dwarfs are expected to become fully convective.

(A color version of this figure is available in the online journal.) 

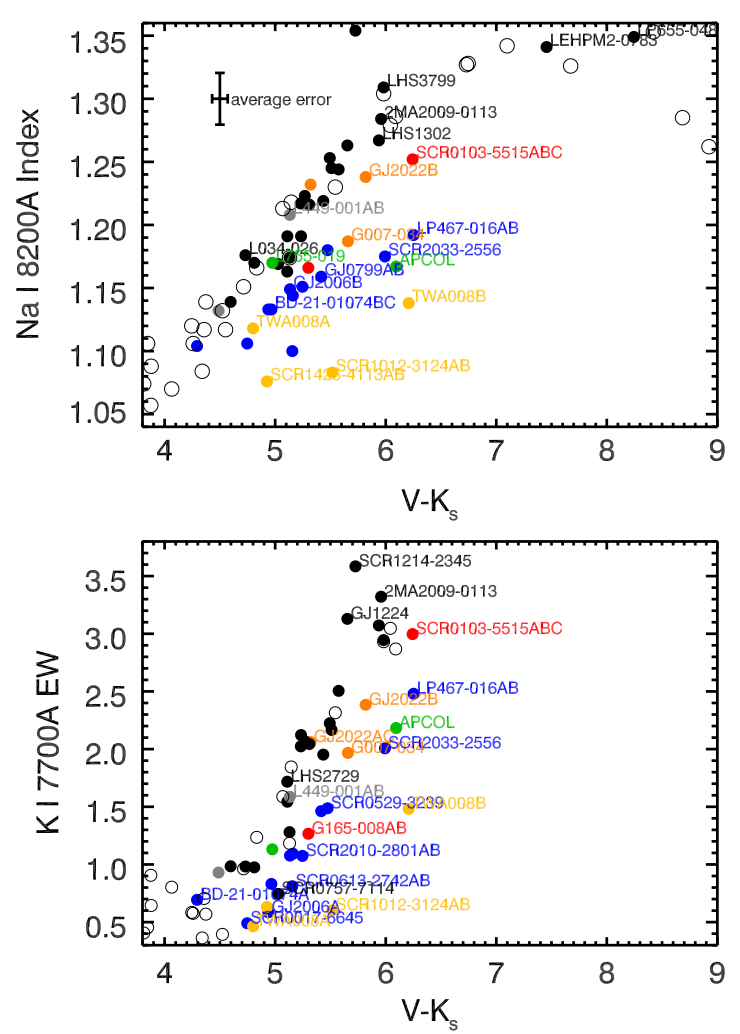

Figure 5. Na I $8183 / 8195 \AA$ doublet gravity index from Lyo et al. (2004b, above) and K I $7699 \AA$ EW (below) vs. $V-K_{S}$ color. Open circles are known main-sequence stars from other RECONS spectroscopic efforts, for comparison. The stars are colored by the association (if any) they appear to belong to: TW Hya (Yellow), $\beta$ Pic (Blue), Tuc-Hor/Columba/Carina (Red), Argus (Green), $\mathrm{AB}$ Dor (Orange), Castor/UMa/Hya (Gray), Field (Black).

(A color version of this figure is available in the online journal.)

$1.5 \mathrm{~m}$ telescope that cannot be reliably corrected for telluric absorption. Thus, there is an additional source of error in our $\mathrm{Na}$ I index measurements, and we are using only one of the $\mathrm{K}_{\mathrm{I}}$ doublet lines (the other is within the atmospheric $A$ band). We conclude that while we see some indication of offsets for young stars in the plots of Figure 5, conclusions of youth using this method with data at this resolution are only tentative.

\subsection{Activity-based Features}

\subsubsection{X-Ray Activity}

As seen in Zuckerman \& Song (2004), X-ray activity remains saturated in M dwarfs even beyond $600 \mathrm{Myr}$. Consequently, $\mathrm{X}$-ray activity is a considered as a necessary but insufficient marker of a star system's youth, and its presence was mostly useful in our sample selection process.

There are, however, eight objects in our sample without X-ray emission. These objects comprise the six previously mentioned systems with no X-ray emission, and two components (GJ 2022B and LP993-115A) of systems with other X-ray detected components. For those targets, we calculate upper limits on their X-ray emission (see Table 6, columns 4 and 5) using the cnts $\mathrm{s}^{-1} \operatorname{arcmin}^{2}$ for the nearest target in the RASS catalog (generally under $2^{\prime}$ distant) as the local background count rate. Even assuming a hypothetical $1 \mathrm{arcmin}$ point spread function, of the eight objects, only TWA $27 \mathrm{AB}$ can potentially have saturated $\mathrm{X}$-ray emission, unless the background count rates for ROSAT are remarkably different over small angular separations. For the special case of NLTT 372 , G 131-26AB is also within the

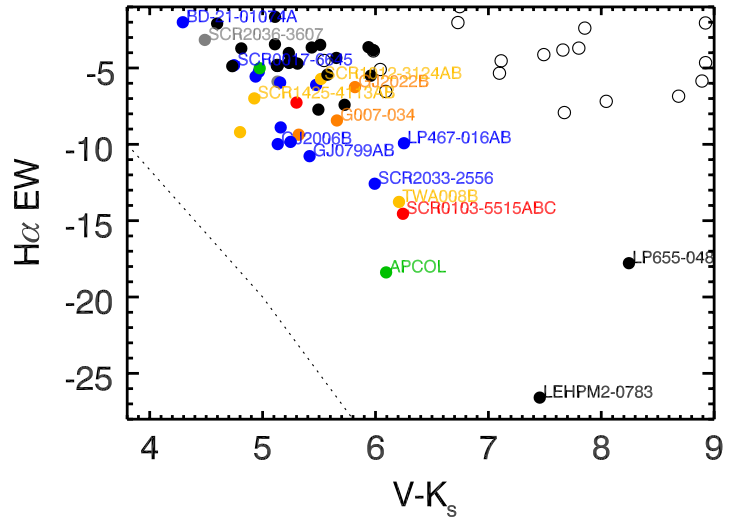

Figure 6. $\mathrm{H} \alpha \mathrm{EW}$ vs. $V-K_{S}$ color. The dashed line is (roughly) the dividing line between Classical T Tauri stars, as defined in White \& Basri (2003). Classical T Tauri stars would appear in the lower left, below the line; none of the stars (even known young stars like RX $1132-2651 \mathrm{AB}=\mathrm{TWA} 8 \mathrm{AB}$ ) are potential Classical $\mathrm{T}$ Tauri stars. The stars are colored by the association (if any) they appear to belong to: TW Hya (Yellow), $\beta$ Pic (Blue), Tuc-Hor/Columba/Carina (Red), Argus (Green), AB Dor (Orange), Castor/UMa/Hya (Gray), Field (Black). Open circles are main-sequence stars from other RECONS spectroscopic efforts.

(A color version of this figure is available in the online journal.)

$25^{\prime \prime}$ radius we used to localize X-ray detections. Although the emission is more likely associated with $\mathrm{G} 131-26 \mathrm{AB}$, if the observed X-ray counts are actually being produced by NLTT 372 , it would be one of the most coronally active stars in our sample.

ROSAT's resolution and accuracy cannot spatially distinguish between targets that are within $\sim 25^{\prime \prime}$ of each other. Consequently, there are a few cases where a multiple system is resolved in the optical and near-infrared but not in X-rays. In these cases, we have combined the $V$ and $K_{S}$ photometry to produce a system bolometric flux. We did not blend NLTT 372 and G 131-26AB, because despite being arcseconds from each other, they are two separate star systems.

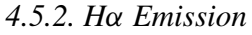

M dwarfs also have saturated $\mathrm{H} \alpha$ emission for long periods of time (West et al. 2008). We have measured this value (Table 6), but it cannot be used to distinguish between the ages of low-mass pre-main-sequence stars. The only useful purpose of $\mathrm{H} \alpha$ emission for low-mass stars is to provide an upper (if present) or lower (if absent) limit on ages, which has been done in Table 7 following the prescription in West et al. (2008). Though we see substantial $\mathrm{H} \alpha$ emission in a few of our stars, none of their emissions reach the White \& Basri (2003) threshold necessary to be considered a T Tauri star (see Figure 6).

\subsubsection{Photometric Variability and Flares}

One of the hallmarks of the T Tauri class of young stars is variability, and this extends into the older non-accreting stars discussed here. Analysis of the relative variability of $\mathrm{M}$ dwarfs (Jao et al. 2011) shows that a typical M dwarf varies by roughly $0.010 \mathrm{mag}$, and statistically significantly more in $V$ and $R(0.013 \mathrm{mag})$ than $I(0.008 \mathrm{mag})$. They also found a statistically significant difference between regular $\mathrm{M}$ dwarfs and their older, metal poor subdwarf cousins (variability $0.007 \mathrm{mag}$, our observational "floor") which points to some combination of age and (possibly) metallicity influencing the amplitude of stellar variability. As seen in Figure 7, M dwarfs with variability higher than 0.020 mag are rare, although many are present among the young stars in this paper. 


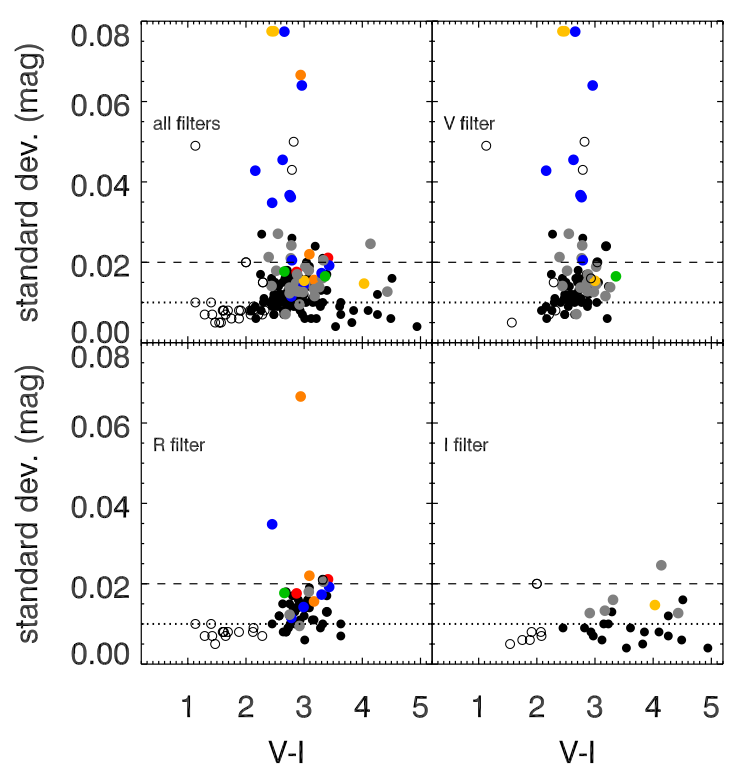

Figure 7. Plot of the relative variability of the stars in this paper (closed symbols, colored as in previous figures), compared to previously published CTIOPI stars (black open symbols), as in Jao et al. (2011). Note the different scale from Jao et al. (2011). Our variability "floor" is $0.007 \mathrm{mag}$, so 0.020 mag clearly indicates a variable star.

(A color version of this figure is available in the online journal.)

While many stars in this paper are known flare stars or UV Ceti variables, only two stars were seen to flare during the course of astrometric observations: TWA 8B and GJ 1207. The relative photometry during the flares is reproduced in Figure 8.

\section{CANDIDATE NEW ASSOCIATION MEMBERS}

Of the 45 systems in this paper, 25 have their first trigonometric parallaxes published here. Fifteen of the 45 systems (including LP 476-207 = HIP 23418) are now new members of the sample of all stars with trigonometric parallax distances within $25 \mathrm{pc}$.

Seven systems are known triples (including GJ 799AB $=$ AT Mic, whose primary is the star GJ $803=$ AU Mic) and 13 are known binaries (Table 10). Twenty-five systems are currently not known to be multiple, though four (NLTT 372, SCR 07577114, LEHP M2-0783, SCR 2033-2556) are strongly suspected to be multiple based on our youth analysis, and one (SCR 0103$5515 \mathrm{ABC}$ ) seems to be too bright even when its multiplicity is taken into account. Thus, these 45 systems include at least 72 objects, for a companion fraction (companion stars/systems) of $60 \%$ and a multiplicity fraction (multiple systems/single systems) of $47 \%$, similar to what was reported by Fischer \& Marcy (1992) for a collection of M0-M3 dwarfs. This high multiplicity rate is to be expected, considering that luminosity (the result of youth or multiplicity) was a defining characteristic of the selection process.

We find 21 potential and/or confirmed members of nearby young associations (Table 7, column 10). Within the sample, we recover 10 members of $\beta$ Pictoris, four members of TW Hydra, two members of Argus, two members of AB Dor, and two young members of unknown associations. In addition, there is one system kinematically consistent with the Ursa Majoris moving group, although by gravity and H-R diagram position it is not distinguishable from a main-sequence star. Particularly noteworthy are the seven new members of $\beta$ Pictoris. Several systems are potentially kinematically consistent with the Hyades, but as
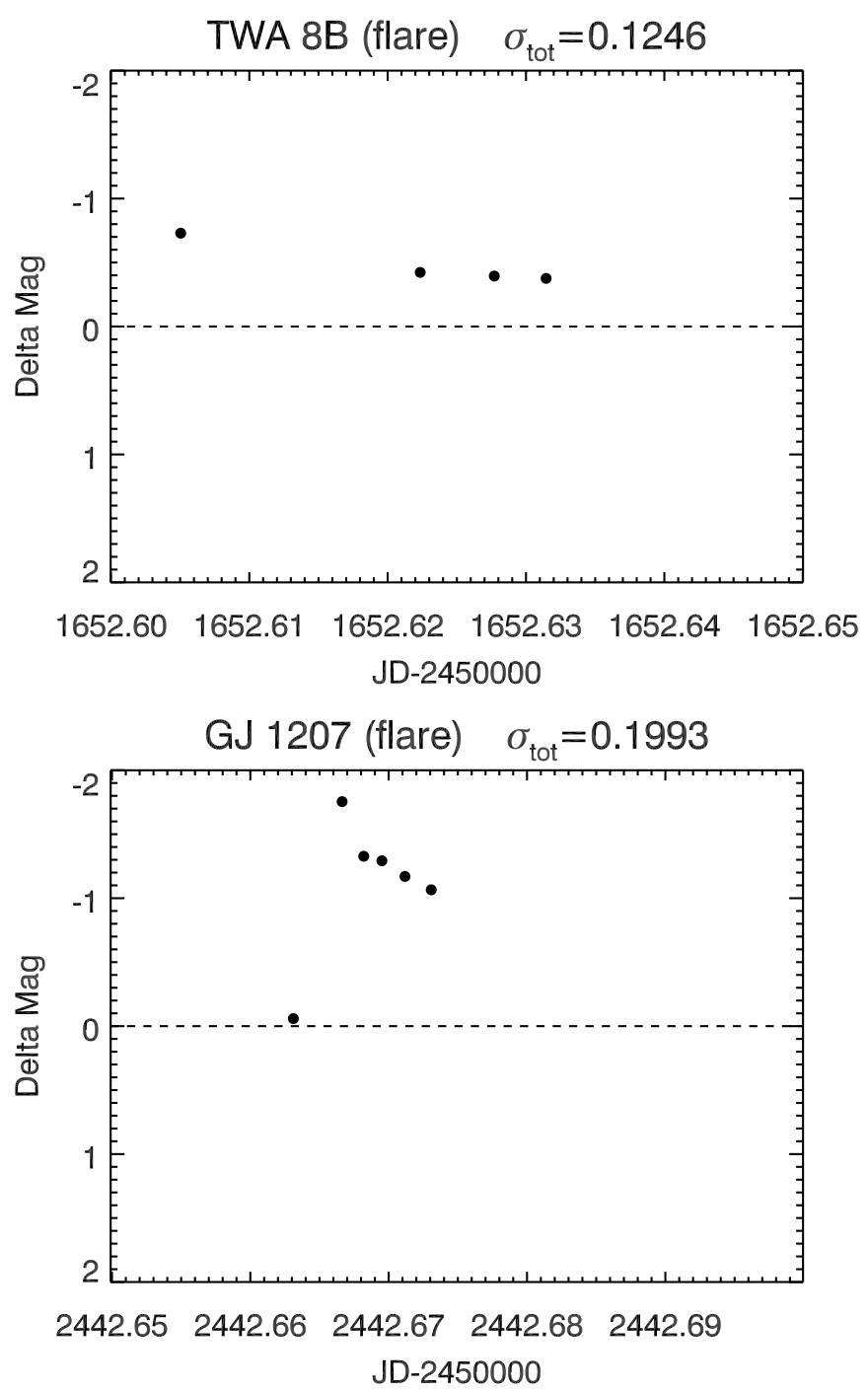

Figure 8. Two large partial flares observed in CTIOPI; note the timescales of the observations and the magnitude spreads; each was observed for less than an hour. We observed the peak of the GJ 1207 flare from 2002 June 17 (which rose in less than 5 minutes), but probably not the peak of the TWA 8B flare from 2000 April 18. Given that each was observed for less than an hour, it is difficult to tell which had a higher peak, was longer-lasting, or had more total energy. The $\sigma_{\text {tot }}$ scatter values given in Tables 2 and 6 are highly biased by these flare events. The zero point of the delta magnitudes were set by the other reference stars in the astrometric solution.

all are more than two tidal radii $(10 \mathrm{pc})$ from the Hyades, and the existence of the Hyades Stream has been brought into question by Famaey et al. (2008), we suspect all potential Hyades memberships are not physical (see Section 5.1).

Several of our objects are likely cooler than the threshold where M dwarfs become fully convective (around $V-K=5.8$ ), and at least three (SCR 0103-5515C, TWA 27A, TWA 27B) are brown dwarfs. It is difficult to make comparative conclusions about such systems, as very few comparably low-mass systems are known in these associations.

For AB Dor, Ursa Major, and Castor, our analysis is more tentative, given that members of those systems are difficult to distinguish from main-sequence stars using isochrones and gravity-sensitive lines, leaving us with only the kinematic analysis. Confirmation of membership in these associations will require measurements of the stars' radial velocities, lithium, chemical abundances (Castro et al. 1999; Barenfeld et al. 2013), 


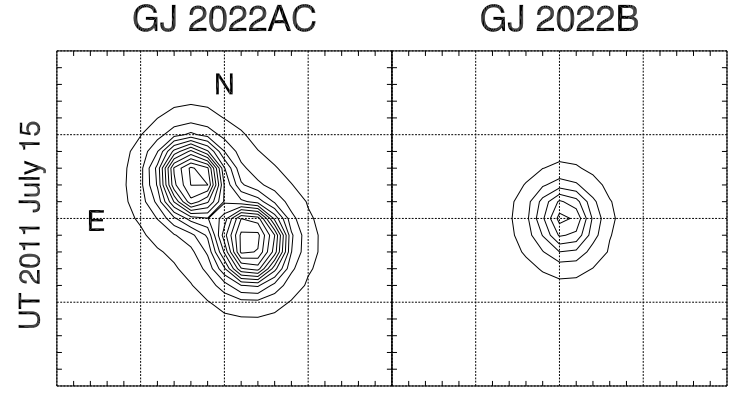

Figure 9. Contour plot of GJ 2022A (south) and C (north) on 2011 July 15 from CTIOPI. GJ 2022B (37'.8 distant) is also plotted as an example single-star PSF, with the same contour intervals. Grid lines are 2".005 apart (5 pixels at the CTIO $0.9 \mathrm{~m})$.

and (in the case of Castor) further study to determine if the moving group genuinely exists at all (Mamajek et al. 2013).

\subsection{Notable Systems (in order of Right Ascension)}

NLTT 372 was originally part of the reference field of the system $\mathrm{G} 131-26 \mathrm{AB}$. It is within the error circle of the ROSAT $\mathrm{X}$-ray source we attribute to $\mathrm{G} 131-26 \mathrm{AB}$, and may either contribute or be the source of those X-rays. It is more luminous than a single star of its photometric colors, and is likely to be a binary.

GJ 2006AB appear to be $\beta$ Pictoris members, based on kinematics, gravity, and their positions relative to the $\beta$ Pictoris isochrone. A spectrum of GJ 2006A was obtained with Very Large Telescope (VLT) instrumentation (L. Malo et al., in preparation) and the radial velocity was determined to be $+8.90 \mathrm{~km} \mathrm{~s}^{-1}$ with $v \sin i$ of $6 \mathrm{~km} \mathrm{~s}^{-1}$, in excellent agreement with that of a predicted $\beta$ Pictoris member.

SCR 0103-5515ABC was resolved as a close triple by Delorme et al. (2013) composed of two M dwarfs, A and B, and a more spatially distant brown dwarf, C (see Table 10). Delorme et al. (2013) and the BANYAN results find it to be a match to Tucana-Horologium, but we find that its kinematics better match the Carina association, and because both associations are supposed to be the same age, we cannot distinguish between them with the other methods. When their photometry is deblended, both components lie above the $\beta$ Pictoris isochrone, implying that they are younger than $\beta$ Pic, even though TucanaHorologium and Carina are both supposed to be older than $\beta$ Pic. The system is undoubtedly young, but more observations are needed to determine if it is a higher-order multiple in TucHor, or something entirely different.

GJ 2022ABC is a hierarchical triple (see Table 10) composed of a wide (37".8) companion (B) to a close (1".8) nearly equalluminosity pair (AC) with a delta magnitude of roughly $\Delta V=$ 0.08 (Jao et al. 2003; Daemgen et al. 2007). The B component is actually the least luminous, though to preserve the historical order of discovery, we continue to refer to it as "B." The $\mathrm{AC}$ component is bright in RASS; there is no corresponding detection of the $\mathrm{B}$ component.

The A and C components are separated by 1".8 (Figure 9). They are separable and (as they are much brighter than the B component) unsaturated on only 31 of the 66 images taken. Those 31 images were taken on 14 nights spanning $11.89 \mathrm{yr}$ and positions were obtained using special extra-sensitive (but less precise) SExtractor settings. A reduction of all three components yields

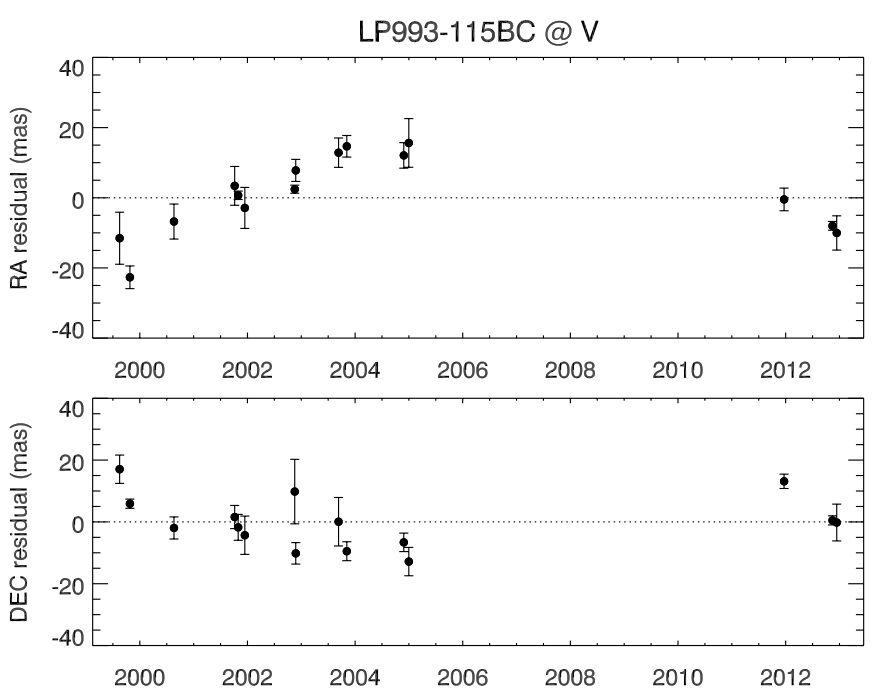

Figure 10. Nightly mean residuals of the LP 993-115BC (LP 993-116AB) parallax fit (after the parallax and proper motion have been removed) show a clear astrometric perturbation in both R.A. and decl. axes. This system is a known binary, but the orbit has not wrapped, and our orbit fit does not converge.

1. GJ 2022A: $\pi=40.91 \pm 5.64$ mas, $\mu=210.5 \pm$

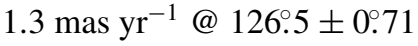

2. GJ 2022B: $\pi=42.12 \pm 3.60$ mas, $\mu=206.2 \pm$ $0.8 \mathrm{mas} \mathrm{yr}^{-1} @ 127.4 \pm 0.46$

3. GJ 2022C: $\pi=46.96 \pm 5.31$ mas, $\mu=200.6 \pm$ $1.2 \mathrm{mas} \mathrm{yr}^{-1} @ 126.8 \pm 0.71$.

This implies a weighted mean parallax of $43.05 \pm 2.63$ mas $(23.2 \pm 1.4 \mathrm{pc})$, which is consistent with our main reduction of $\mathrm{B}$ in Table 3 using 66 frames $(38.80 \pm 2.13$ mas, $25.8 \pm 1.4 \mathrm{pc})$ and with membership in the AB Dor association. Formally, we are using the parallax of the B component alone as our system parallax in Table 4 due to the lower precision of the results for all three components.

LHS 1302 is potentially a kinematic match for the $\sim 30 \mathrm{Myr}$ old Columba association, but its gravity (Figure 5) and isochrones (Figure 4) suggest it is a field object.

$L P$ 993-115 (A)/LP 993-116AB (BC). We detect the astrometric orbital motion of the BC pair (Figure 10). Our attempt to fit an orbit did not converge, and the astrometry in Table 3 was calculated without compensating for orbital motion. This triple system appears to be composed of field stars, and only the BC components have X-ray emission.

LP 476-207ABC was observed by Hipparcos as HIP 23418; the resulting parallax was of poor quality due to erroneous coordinates in the input catalog (Perryman et al. 1997) and the resulting poor available astrometry. Our new result (24.6 \pm $1.3 \mathrm{pc}$ ) is significantly closer and higher precision than the old (Perryman et al. 1997; $32.1 \pm 8.8 \mathrm{pc}$ ) and revised (van Leeuwen $2007 ; 33.2 \pm 10.5$ pc) Hipparcos values, but the system is still in $\beta$ Pic.

$B D-21^{\circ} 1074 A B C$ is a known member of the $\beta$ Pic association (Torres et al. 2008; Malo et al. 2013). The A component was not originally targeted for parallax measurement and was saturated in many early images; the parallax result in Table 3 is of lower precision.

The BC component was observed by the HST FGS Interferometer on 2008 December 18, and resolved (Figure 11) in both axes, with details in Table 10. The measured position angle is discrepant with the current Washington Double Star cata-

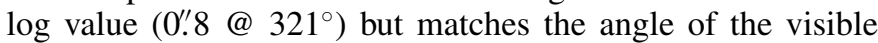




\section{$\mathrm{BD}-21^{\circ} 1074 \mathrm{BC}$}
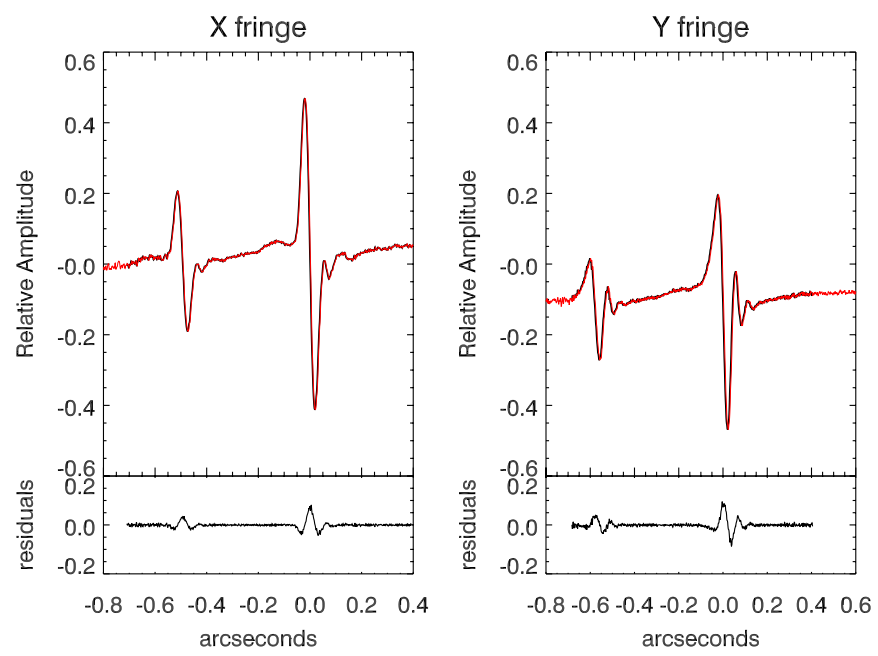

Figure 11. $X$-axis (left) and $Y$-axis (right) Hubble Space Telescope Fine Guidance Sensor preliminary results for BD- $21^{\circ} 1074 \mathrm{BC}$. Both axes show the pronounced presence of a second component (note that the axes shown here are not R.A. and decl., but rather the FGS 1r axes at the time of the observation. The quoted position angles elsewhere have been corrected for spacecraft roll angle). Lower panel shows the residuals to the fit; the subtraction is not perfect.

(A color version of this figure is available in the online journal.)

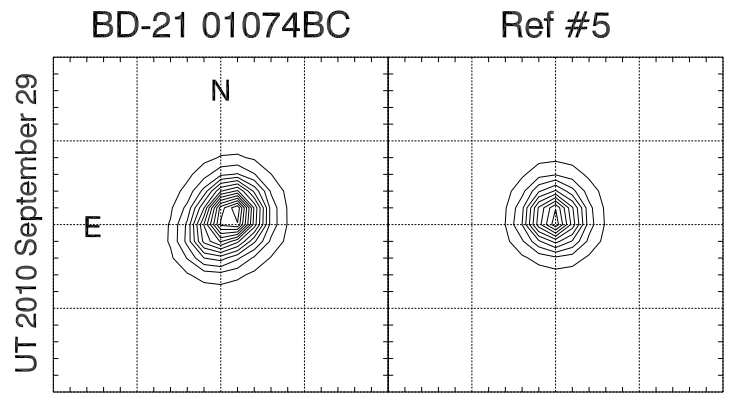

Figure 12. Contour plot of BD- $21^{\circ} 1074 \mathrm{BC}$ on 2010 September 29 from CTIOPI data; the SE elongation is probably the $\mathrm{C}$ component, despite WDS claiming the position angle is $321^{\circ}$. The nearest reference star (5) is plotted as a representative single-star PSF, with $4 \times$ smaller contour intervals. BD-2 $1^{\circ} 1074 \mathrm{~A}$ is saturated on this frame and not shown. Grid lines are 2".005 apart (5 pixels at the CTIO $0.9 \mathrm{~m})$.
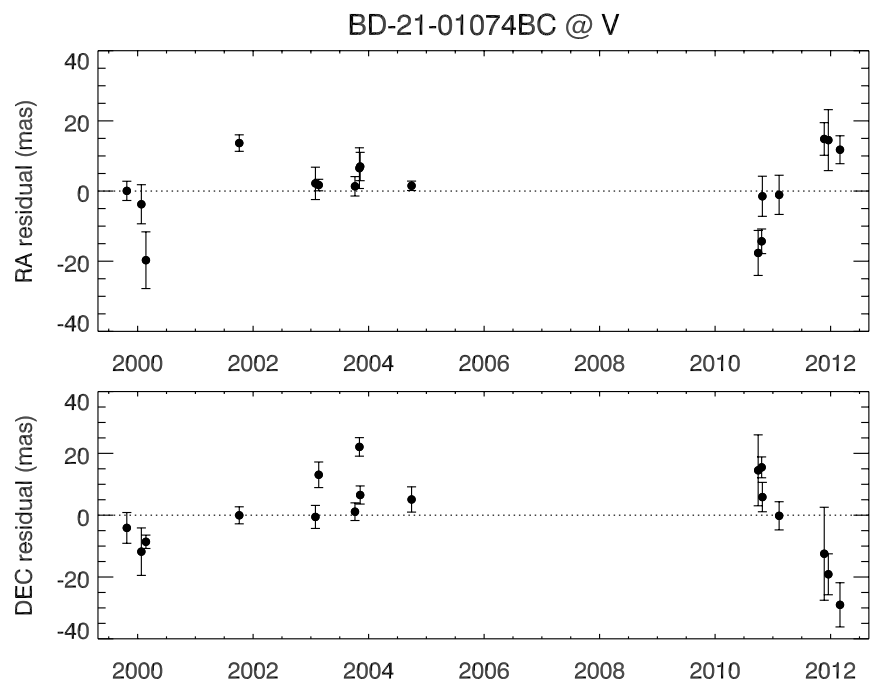

Figure 13. Nightly mean residuals of the parallax fit (after the parallax and proper motion have been removed) show a clear astrometric perturbation in both R.A. and decl. axes. This system is a known binary, but the orbit has not wrapped, and our orbit fit does not converge.
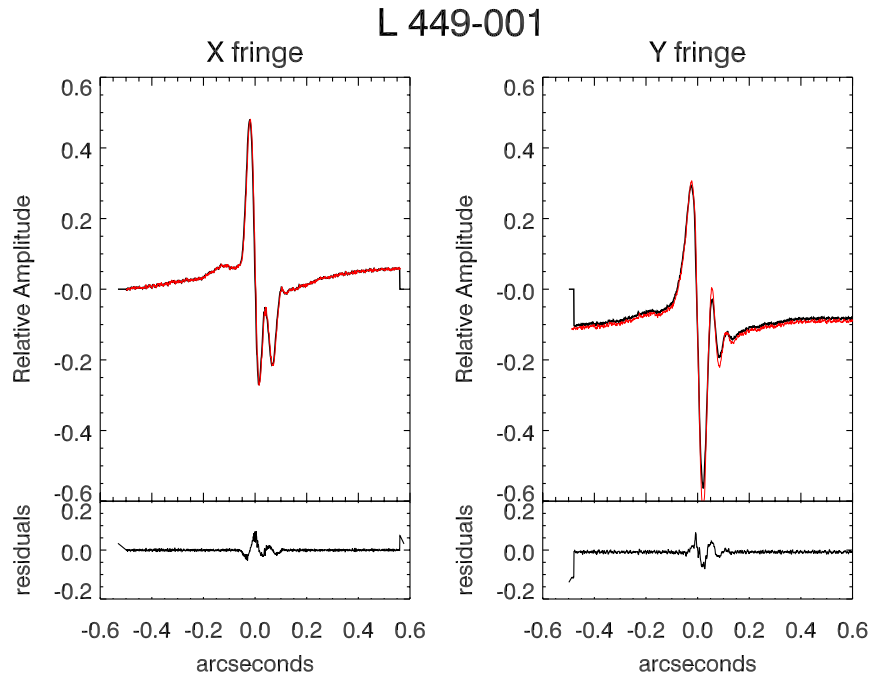

Figure 14. $X$-axis (left) and $Y$-axis (right) Hubble Space Telescope Fine Guidance Sensor preliminary results for $\mathrm{L} 449-1 \mathrm{AB}$. The $X$-axis "S-curve" of the Fine Guidance Sensor shows a second dip to the right of the main one, revealing a companion. The residuals to the fit (bottom) demonstrate that the companion is not readily resolved in the $Y$-axis S-curve.

(A color version of this figure is available in the online journal.)

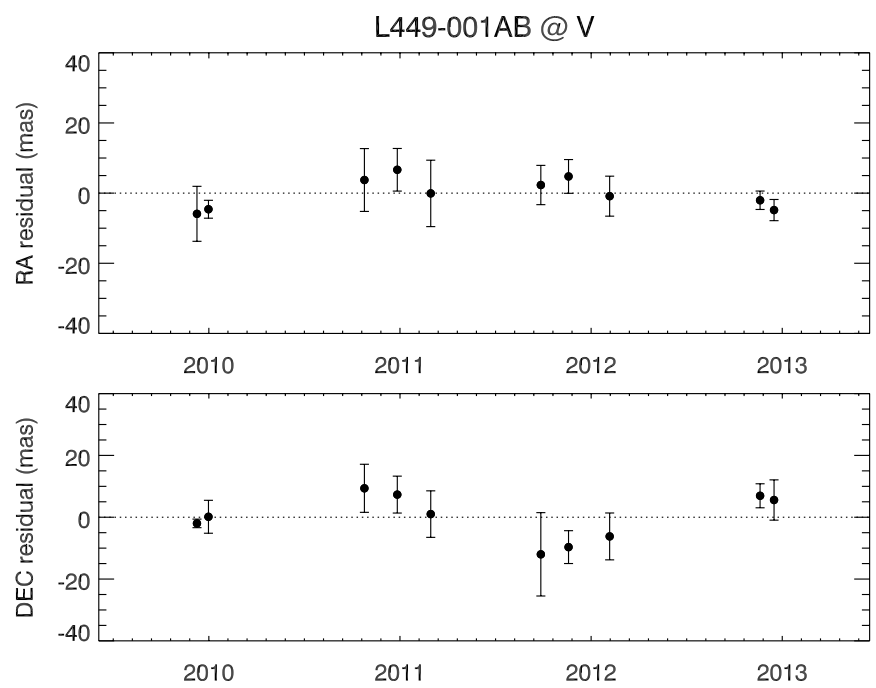

Figure 15. Nightly mean residuals of the parallax fit (after the parallax and proper motion have been removed) show an astrometric perturbation in both R.A. and decl. axes. The CTIOPI data may cover a full orbit, but the astrometric signal is weak.

elongation of the BC point spread function from CTIOPI data, as seen in Figure 12. We see an astrometric binary signal in our astrometry, as shown in Figure 13.

$L 449-1 A B$ was identified as an active star by Scholz et al. (2005). This system was on the HST Cycle 16B FGS proposal, and was resolved into two stars on 2008 December 3 (Figure 14) with $\Delta \mathrm{F} 583 \mathrm{~W}=0.95 \mathrm{mag}$ (see Table 10). The astronomic data displays what may be a very weak perturbation (Figure 15) due to orbital motion, but it is not convincing. By H-R diagram isochrones and gravity indices, this system is indistinguishable from main-sequence stars. The system's kinematics are a potential match for the Ursa Major moving group, which given the probable age of $500 \mathrm{Myr}$ (King et al. 2003) again suggests that the components may be nearly zero-age main sequence (ZAMS).

LHS 1358 has kinematics consistent with the Hyades stream. Various authorities (e.g., Famaey et al. 2008) dispute the 

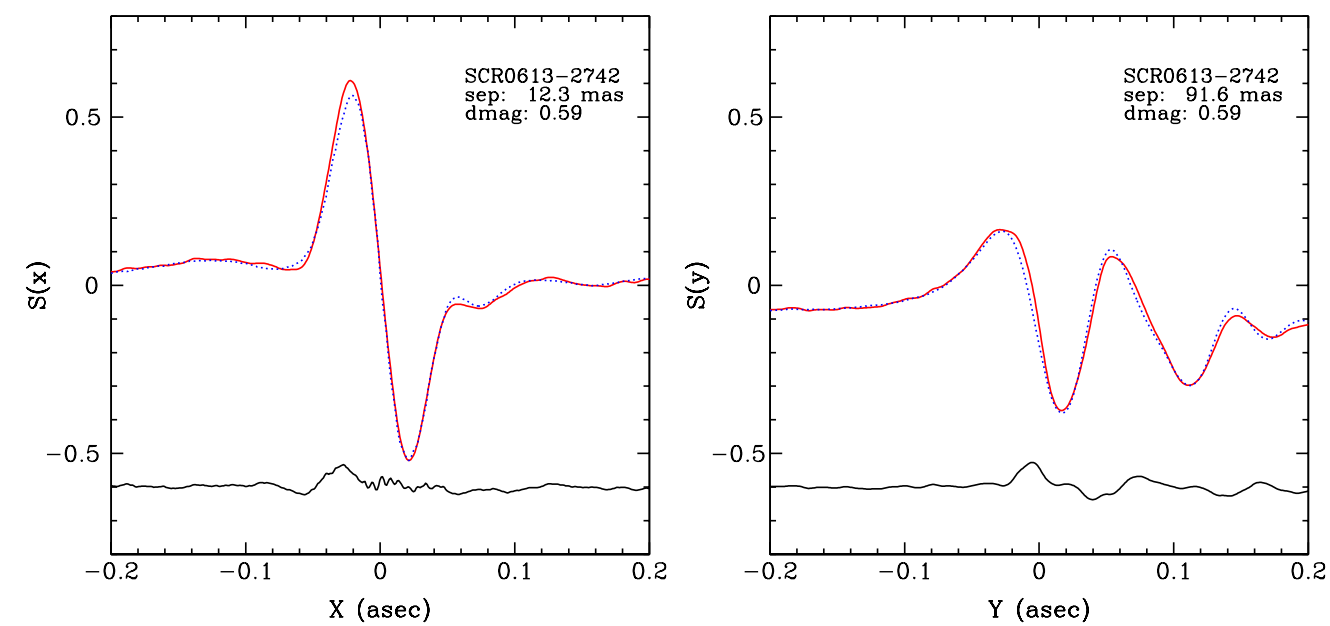

Figure 16. $X$-axis (left) and $Y$-axis (right) Hubble Space Telescope Fine Guidance Sensor results for SCR 0613-2742AB. The $Y$-axis "S-curve" of the Fine Guidance Sensor shows a second dip to the right of the main one (compare to the axes of L 449-1AB, Figure 14), revealing a companion. The companion is also barely resolved at \pm 12 mas (near the limit of FGS's capabilities) in the $X$-axis, though this is not visibly apparent, and carries a sign ambiguity.

(A color version of this figure is available in the online journal.)
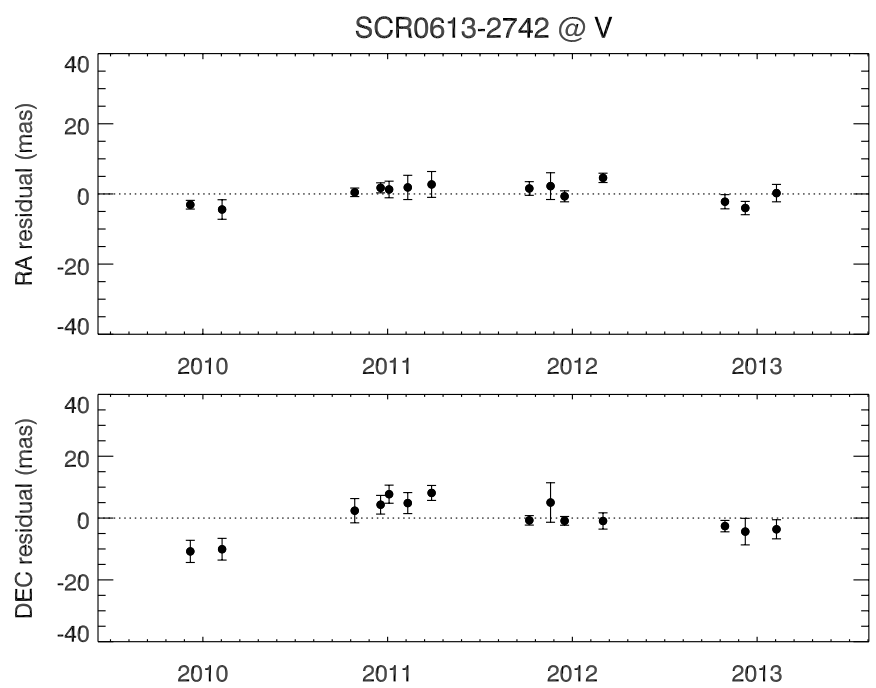

Figure 17. Nightly mean residuals of the parallax fit (after the parallax and proper motion have been removed) show an astrometric perturbation in both R.A. and decl. axes. We have resolved this binary with FGS, but we do not yet have an orbit.

existence of the stream as a real kinematic entity; at only $15.3 \pm 0.5 \mathrm{pc}$ from the Sun, LHS 1358 is a minimum of $30 \mathrm{pc}$ ( 3 tidal radii) from the Hyades cluster center, and therefore the identification is not physical.

$S C R \quad 0613-2742 A B$ is the lowest proper motion system $\left(11.2 \pm 1.0\right.$ mas $\left.\mathrm{yr}^{-1}\right)$ thus far observed on CTIOPI, with a transverse velocity of $1.6 \mathrm{~km} \mathrm{~s}^{-1}$. The system is a binary, observed on 2008 December 4 and resolved into two stars (Figure 16, Table 10) by FGS, and is also detected as a binary by our FEROS spectroscopy. We see orbital motion in the CTIOPI astrometric residuals of this star (Figure 17) and have removed that orbital motion ${ }^{19}$ from our astrometric results (Riedel et al. 2010), but we do not have data on a full orbit. Based on the FEROS spectroscopy, we see no Li $6708 \AA$

19 The orbit that was fit assumes a $4.67 \mathrm{yr}$ orbit starting at $T_{0}$ of 2010.803 Julian Years, with photometric semimajor axis 0.54 arcsec, inclination $89.9 \mathrm{deg}$, eccentricity of 0.9999 , a longitude of the ascending node of $201.3 \mathrm{deg}$, and longitude of periastron of $90.3 \mathrm{deg}$. While it removes the orbital bias from our astrometric data, we do not consider the orbit correct. doublet absorption. This is expected for $\beta$ Pictoris members near the lithium depletion boundary (Yee \& Jensen 2010).

The position and proper motion of SCR 0613-2742AB, near the convergent point of the $\beta$ Pic association, are extremely similar to those of 2MASS J06085283-2753583 (Cruz et al. 2003; Rice et al. 2010), a brown dwarf $\beta$ Pic member. The parallax of $2 \mathrm{M} 0608$ (32.0 \pm 3.6 mas, $31.3 \pm 3.5$ pc; Faherty et al. 2012) is also very similar to SCR0613 (34.0 $\pm 1.0 \mathrm{mas}, 29 \pm 0.9 \mathrm{pc})$. The two systems are separated by 3529" @ 78.6, which yields a minimum projected separation of $100 \mathrm{kAU}(\approx 0.5 \mathrm{pc})$. It is difficult to compare their proper motions- $(+8.9,+10.7) \pm$

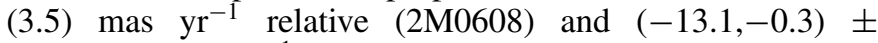
$(11.6,15.5)$ mas $r^{-1}$ absolute (SCR0613)—given the large uncertainties involved, save that each are marginally consistent with both zero and each other.

Meanwhile, the binding energy, $U_{g}=\left(-G M_{1} M_{2} / r\right)=$ $-1.28 \times 10^{33} \mathrm{~J}$ using the conservative estimates that the two components of SCR0613 have a combined mass of $0.5 M_{\odot}$, and 2M0608 is $0.015 M_{\odot}$. This binding energy is lower than any of the extremely wide systems studied in Caballero (2009), and it is probable that these stars are not gravitationally bound. It is possible that this was a bound system at one point (like AU/AT Mic $=$ GJ 803/799AB, below), but is not any longer.

SCR 0757-7714 is overluminous by 2 mag, plotting near the $\beta$ Pic isochrone on the H-R diagram in Figure 4, and has low surface gravity based on its K I measurement, but not Na I. Surprisingly, it shows no other signs of youth or membership in any known association - in fact, it has no X-ray emission, and is the only star under consideration with $\mathrm{H} \alpha$ in absorption. Thus, the star's elevation on the H-R diagram is most likely due to unresolved multiplicity.

$L$ 34-26 is a potential kinematic match to the Ursa Major moving group, but its measured radial velocity $\left(+0.9 \mathrm{~km} \mathrm{~s}^{-1}\right.$, no error; Torres et al. 2006) is most likely discrepant with the best-fit Ursa Major moving group radial velocity, $+6.6 \mathrm{~km} \mathrm{~s}^{-1}$.

$S C R \quad 1012-3124 A B$ is a close $\sim 1^{\prime \prime}$ binary (Figure 18 and Table 10). There are a few epochs where the $\mathrm{B}$ component (to the west of the A component) can be seen, but for the most part the two components are blended in our astrometric observations.

Radial velocity measurements with the VLT-UT1 CRyogenic high-resolution infraRed Echelle Spectrograph (CRIRES) presented in L. Malo et al. (in preparation) confirm the star's 


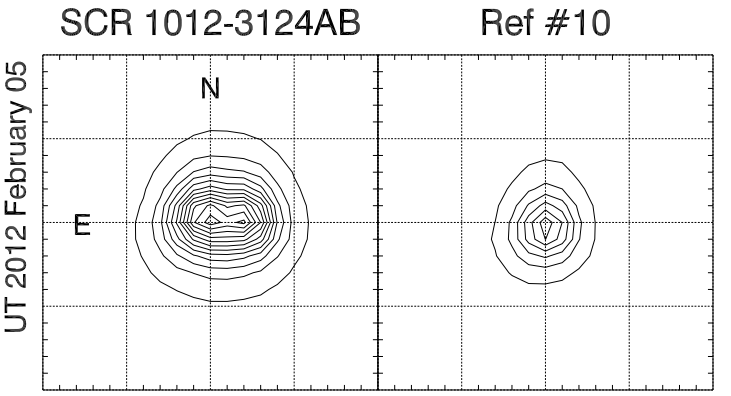

Figure 18. Positions of SCR 1012-3124A (E) and B (W) on a rare night when the binary is detectable in CTIOPI data. Reference star 10 is also plotted for a comparison of a single-star PSF from the same night, with the same contour intervals. Grid lines are 2'.005 apart ( 5 pixels at the CTIO $0.9 \mathrm{~m}$ ).

multiplicity, and yield radial velocities of $14.69 \pm 0.53 \mathrm{~km} \mathrm{~s}^{-1}$ and $14.43 \pm 0.75 \mathrm{~km} \mathrm{~s}^{-1}$, for $\mathrm{A}$ and $\mathrm{B}$, respectively. The $v \sin i$ measurements (A: $15.52 \pm 2.01 \mathrm{~km} \mathrm{~s}^{-1}$; B: $20.40 \pm 2.58 \mathrm{~km} \mathrm{~s}^{-1}$ ) are also indicative of youth, where Reiners et al. (2009) considers $v \sin i>20 \mathrm{~km} \mathrm{~s}^{-1}$ the minimal condition for a "fast rotator."

While it is outside the normal spatial bounds (Torres et al. 2008) of the TW Hydra association (its R.A. is slightly lower than that of TWA 21 , at $10^{\mathrm{h}} 13^{\mathrm{m}}$ ), its $U V W$ kinematics are consistent with TW Hydra, as is its deblended isochrone position (Figure 4). Its gravity measurements (Figure 5) indicate it is extremely young.

TWA $8 A B$ was listed by Jao et al. (2003) as RXJ1132-264AB and was not included in the TW Hydra analysis of Weinberger et al. (2013); our information is consistent with their conclusions based on other members of TW Hydra in that analysis.

$G 165-8 A B$ appears to be between the ages of $\beta$ Pictoris and AB Doradus based on its gravity measurement and deblended $\mathrm{H}-\mathrm{R}$ diagram positions. There are two highly discrepant radial velocities published for this system, $+8 \pm 0.1 \mathrm{~km} \mathrm{~s}^{-1}$ (Montes et al. 2001) and $-7.5 \pm 6.5 \mathrm{~km} \mathrm{~s}^{-1}$ (Gizis et al. 2002). We would normally choose the former value due to its higher precision, but the kinematics derived using that value agree with no known association. Ignoring radial velocities altogether, we find bestfit matches to the Tucana-Horologium $(\gamma=0.58$, best-fit RV $\left.-6.2 \mathrm{~km} \mathrm{~s}^{-1}\right)$, Carina $\left(\gamma=0.62\right.$, best-fit RV $\left.-10.4 \mathrm{~km} \mathrm{~s}^{-1}\right)$, and Columba $\left(\gamma=2.40\right.$, best-fit $\left.\mathrm{RV}-12.5 \mathrm{~km} \mathrm{~s}^{-1}\right)$ associations, all with estimated ages of $30 \mathrm{Myr}$. It seems likely that the Gizis et al. (2002) radial velocity is accurate, and the uncertainty is accounting for orbital motion of the binary. Using the latter radial velocity, the best match is to Carina, though BANYAN favors Columba.

Unusually, this system is a northern hemisphere target well outside the published spatial boundaries of all three associations. This suggests that either there is a fourth 30 Myr old association nearby, possibly containing two other northern hemisphere objects thought to be members of the $30 \mathrm{Myr}$ old Columba association: HR 8799 (Marois et al. 2008; Baines et al. 2012) and $\kappa$ And (Carson et al. 2013), or that the existing assumptions about the boundaries of the known associations are incorrect.

$S C R \quad 1425-4113 A B$ is a curious system. High-resolution ESPaDOnS spectra of this target show that it is a spectroscopic binary, and an extremely rapid rotator $(v \sin i=95.0 \pm$ $\left.8.1 \mathrm{~km} \mathrm{~s}^{-1}\right)$, with a radial velocity of $\left(-1.2 \pm 1.3 \mathrm{~km} \mathrm{~s}^{-1}\right)$. Its lithium EW was also measured at $595 \pm 20 \mathrm{~m} \AA$, confirming its youth. The deblended magnitudes of both of its components place them above (though consistent with) the TW Hydra isochrone on an H-R diagram, and the gravity measurements
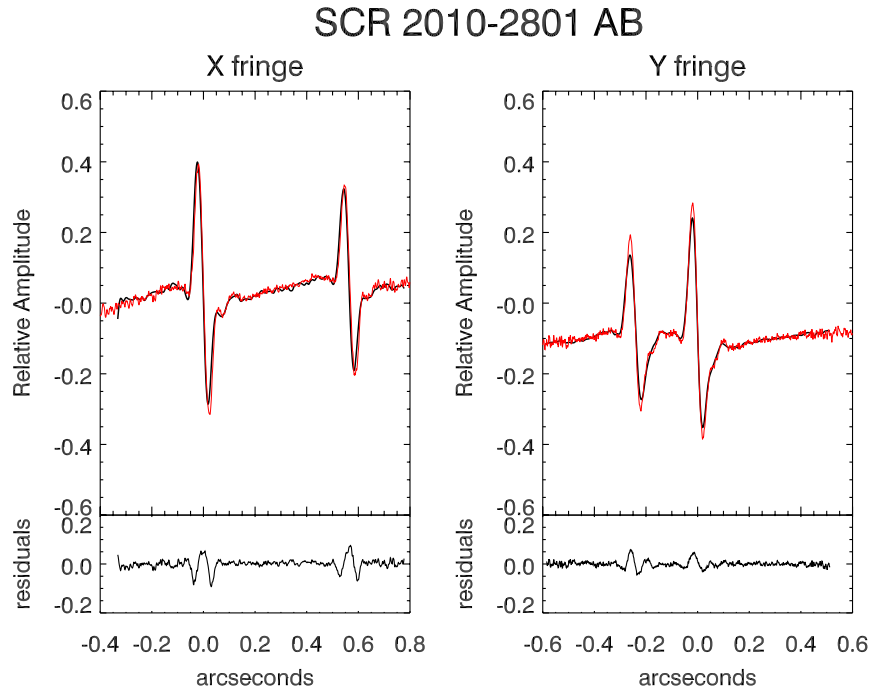

Figure 19. $X$-axis (left) and $Y$-axis (right) Hubble Space Telescope Fine Guidance Sensor results for SCR 2010-2801AB. The dual "S-curves" of the two components, originally resolved by Beuzit et al. (2004), are easily visible. The residuals, on the bottom, demonstrate the quality of the fit.

(A color version of this figure is available in the online journal.)

(which should not be affected by multiplicity) place it at lower surface gravity than TWA 8AB and SCR 1012-3124AB (though still within the errors).

Our kinematics find it to be a much better fit to $\beta$ Pictoris $(\gamma=0.59)$ than TW Hydra $(\gamma=6.46)$, but BANYAN finds $75 \%$ probability of it being a TW Hydra member, compared with $24 \%$ probability that it is actually a $\beta$ Pictoris member. It is additionally outside the normal spatial boundaries of TW Hydra (being a full hour of R.A. "higher" than the highest current member, TWA 18 , at $13^{\mathrm{h}} 21^{\mathrm{m}}$ R.A.). We nevertheless consider it as a potential TW Hydra member because with its extremely high luminosity, low surface gravity, and position relatively close to TW Hydra, it either must be an outlying member of TW Hydra or something equally young.

$S C R 2010-2801 A B$ was found to be a binary by Bergfors et al. (2010). The system was observed with HST-FGS on 2009 April 26, as seen in Figure 19. The resulting separation agrees with the separation published in Bergfors et al. (2010).

LEHPM2-0783 has X-ray emission and the strongest $\mathrm{H} \alpha$ emission in the sample, despite its cool temperatures. It lies above the main sequence, though it is too red to be seen on Figure 4. It is, however, not a potential kinematic match to any known young association. It may be younger than a typical field star, but we have no reason to suspect it is anything more than an unresolved binary.

$L 755-19$ is kinematically consistent with both Castor and Argus. It lies within the upper main-sequence locus, above the AB Doradus isochrone, which would be more consistent with the Argus association (younger than AB Dor) or a main-sequence binary than the Castor moving group (older than AB Dor). L 755-19 is a hotter star than AP Col (Riedel et al. 2011), and its surface gravity is comparable with either field stars or Argus-age objects. A radial velocity would go a long way toward determining the status of this star, given that the best-fit Argus $\mathrm{RV}$ is $-25.4 \mathrm{~km} \mathrm{~s}^{-1}$, and the best-fit Castor RV is $-9.3 \mathrm{~km} \mathrm{~s}^{-1}$, and a Castor/main-sequence assignment would imply it is a binary.

SCR 2033-2556 is consistent with being a member of $\beta$ Pictoris, but its high luminosity suggests it is an unresolved binary. 


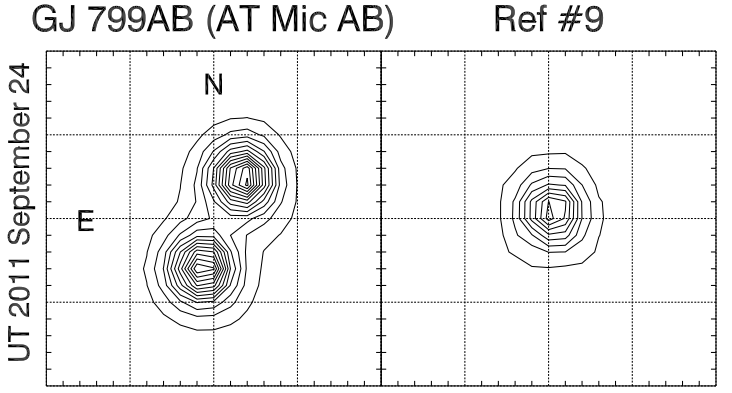

Figure 20. GJ $799 \mathrm{~A}(\mathrm{~N})$ and B (S) on 2011 September 24 from CTIOPI data. The nearest reference star (9) is plotted as an example of a single-star PSF, with $800 \times$ smaller contour intervals. Grid spacings are 2 ". 05,5 pixels at the CTIO $0.9 \mathrm{~m}$.

Observations with ESPaDOnS (L. Malo et al., in preparation) suggest a lithium EW of $510 \mathrm{~mA}$, confirming the star's youth.

GJ $803(A) / G J 799 A B(B C)$ is one of the nearest young systems (see Table 10) to the Sun, and a prototypical member of the $\beta$ Pic association (Barrado y Navascués et al. 1999). AU Mic (unobserved by CTIOPI) is known to have a dust disk, and several authors note that the AU-AT Mic separation is very large (at least $0.23 \mathrm{pc}$ ) and "must be very fragile and will soon be torn apart by third bodies" (Caballero 2009). This system is a well-established member of the 10 pc sample with parallaxes from Hipparcos and the Yale Parallax Catalog (van Altena et al. 1995); our results agree with the published values.

In our first epoch (2003 July 9) GJ 799 A and B were separated by $2^{\prime \prime} 8$ @ $171^{\circ}$; in our final epoch (2012 July 31, Figure 20) they were separated by $2^{\prime \prime} .3 @ 153^{\circ}$.

GJ $1284 A B$ is identified as a potential member of Columba by kinematic analysis; however, Torres et al. (2006) identified it as a spectroscopic binary, which implies it is a system of main-sequence stars, and the kinematic match is spurious.

\section{CONCLUSIONS}

We have identified 21 young systems, of which 14 are new, and six-LP 467-16AB, G 7-34, L 449-1AB, G 165-8AB, L 755-19, and SCR 2033-2556-are within 25 pc of the Sun, as outlined in Table 6 . This constitutes $6 \%$ of all known $\beta$ Pictoris members, and adds two new TW Hydra members that are outside the currently understood boundaries of the system. We also further increase the number of nearby Argus and AB Doradus members. Additionally, the enormous number of multiples will be enormously beneficial to studies of stellar masses for young stars; there are less than 10 masses for $\mathrm{M}$ dwarf stars less than 10 Myr old (Mathieu et al. 2007) and therefore considerable discrepancies in the various predicted evolutionary tracks of low-mass stars; this paper includes three new spectroscopic binaries in TW Hydra and $\beta$ Pictoris where every component is less than $0.5 M_{\odot}$.

The most interesting results of this analysis are the contradictions with expected young star behavior. Six systems in the sample are clearly overluminous but none of their components have RASS X-ray detections. Three of them-SCR 0103-5515ABC, SCR 1012-3124AB, and SCR 1425-4113AB-show H $\alpha$ emission in low-resolution optical spectra; all three are definite young systems, and all three are multiples. The fourth target, SCR 0757-7114, shows no signs of activity at all and is more likely a triple or quadruple system. The remaining two objects-NLTT 372 and TWA $27 \mathrm{AB}$ - were not observed spectroscopically, so we cannot comment on their $\mathrm{H} \alpha \mathrm{EW}$ (though Gizis \& Bharat 2004 recounts wildly varying $\mathrm{H} \alpha$ emission from TWA 27AB).

We checked the XMM-Newton and Chandra observing logs to see if any of the six systems were observed by higher-sensitivity equipment, and found a Chandra observation of TWA 27AB. That observation formed the basis of the work by Gizis \& Bharat (2004), who report no detected X-ray flux in a $50 \mathrm{ks}$ observation, and provided only an upper limit. Given that TWA 27AB was the only target that might have had saturated X-rays given the upper limit from RASS photometry, we can fairly conclusively state that none of these systems exhibit X-ray emission.

It is not clear how these six systems (or GJ 2022B and LP 993-115) exhibit such low X-ray activity at such young ages, except to point out that $\mathrm{H} \alpha$ emission is thought to be produced in the chromosphere, and X-ray activity originates in the corona; Riaz et al. (2006) and West \& Basri (2009) have noted that $\mathrm{H} \alpha$ emission does not correlate well with X-ray activity. Gizis \& Bharat (2004) suggest the enormous (and variable) $\mathrm{H} \alpha$ emission they have compiled from TWA 27AB, combined with its lack of X-ray activity, implies that the corona and chromosphere are quiet, and the $\mathrm{H} \alpha$ activity actually comes from accretion. Given that SCR 1012-3124AB and SCR 1425$4113 \mathrm{AB}$ are both suspected members of the same $8 \mathrm{Myr}$ old TW Hydra association, it is not inconceivable for them to have disks, though more observations will be needed. Explaining a disk around SCR 0103-5515ABC is more difficult, considering that as a member of Tucanae-Horologium it is expected to be $\sim 30$ Myr old, though with its high luminosity for the two stellar components, we do have evidence that it may be a younger system.

The issue of X-ray emission highlights the reason why we favor a checklist-style or multi-parameter approach to youth, such as those used by Shkolnik et al. (2009, 2011). None of the indicators of youth are infallible-if we were to strictly require X-ray saturation, we would have missed both new potential TW Hydra members, and have only noticed the AB Doradus member GJ 2022B (Shkolnik et al. 2012) on the strength of its companions-but together they can provide a clearer and more robust picture of youth. Equally important, these stars are variable, and some number of them will be caught by any given survey in a transient inactive state.

We also have a small assortment of stars that do not quite fit into our current knowledge of young clusters: Both of the putative new TW Hydra members (SCR 1012-3124AB and SCR 1425-4113AB) lie outside the spatial bounds of the TW Hydra association members defined in Torres et al. (2008); $\mathrm{G} 165-8 \mathrm{AB}$ is clearly young, but lies outside the spatial and kinematic boundaries of all known suitably young associations. SCR 0103-5515A and B are each too luminous to be members of Tucana-Horologium or Carina unless both are further close multiples (each lies $\sim 0.4$ mag above the $\beta$ Pictoris isochrone, as do LP 476-207B, SCR 2010-2801A and B, and SCR 0017-6645, though in those cases our deblending method may be at fault, see Section 4.2.1). Ultimately, further study is needed to determine the true extent of these young associations, and determine what manner of processes have brought the associations to their current state.

The RECONS effort is supported primarily by the National Science Foundation through grants AST 05-07711 and AST 0908402, and was supported for a time through NASA's Space Interferometry Mission. Observations were initially made possible by NOAO's Survey Program and have continued via the 
SMARTS Consortium. This research has made use of the SIMBAD database and the VizieR catalogue access tool, operated at CDS, Strasbourg, France; NASA's Astrophysics Data System; the SuperCOSMOS Science Archive, prepared and hosted by the Wide Field Astronomy Unit, Institute for Astronomy, University of Edinburgh, which is funded by the UK Science and Technology Facilities Council; the Washington Double Star Catalog maintained at the U.S. Naval Observatory; and the NStars project. This publication makes use of data products from the Two Micron All Sky Survey, which is a joint project of the University of Massachusetts and the Infrared Processing and Analysis Center/California Institute of Technology, funded by the National Aeronautics and Space Administration and the National Science Foundation, and the AAVSO Photometric All-Sky Survey (APASS), funded by the Robert Martin Ayers Science Fund. This research has made use of the Washington Double Star Catalog maintained at the U.S. Naval Observatory. D.R.R. acknowledges support from project BASAL PFB-06 of CONICYT, a Joint Committee ESO-Government of Chile grant, and FONDECYT grant 3130520. S.C.B and E.L.R acknowledge support from NASA award 11-ADAP11-0169.

The authors also wish to thank Dr. Brian Mason for supplying the orbit-fitting code; Dr. Stella Kafka for the CTIO 4.0 m spectra, Nikhil van der Klaauw, Sean Samaroo, Emmett Goodman-Boyd, Jonathan Gagné and Dr. Inseok Song for useful discussions, and the staff of the Cerro Tololo Inter-American Observatory, particularly Edgardo Cosgrove, Arturo Gomez, Alberto Miranda, and Joselino Vasquez, for their help over the years.

\section{REFERENCES}

Adams, J. D., Stauffer, J. R., Monet, D. G., Skrutskie, M. F., \& Beichman, C. A. 2001, AJ, 121, 2053

Allers, K. N., Jaffe, D. T., Luhman, K. L., et al. 2007, ApJ, 657, 511

Baines, E. K., White, R. J., Huber, D., et al. 2012, ApJ, 761, 57

Baraffe, I., Chabrier, G., Allard, F., \& Hauschildt, P. H. 1998, A\&A, 337, 403

Baraffe, I., Chabrier, G., Allard, F., \& Hauschildt, P. H. 2002, A\&A, 382, 563

Barenfeld, S. A., Bubar, E. J., Mamajek, E. E., \& Young, P. A. 2013, ApJ, 766,6

Barrado y Navascues, D. 1998, A\&A, 339, 831

Barrado y Navascués, D., Stauffer, J. R., Song, I., \& Caillault, J. 1999, ApJL, 520, L123

Benedict, G. F., McArthur, B., Chappell, D. W., et al. 1999, AJ, 118, 1086

Bergfors, C., Brandner, W., Janson, M., et al. 2010, A\&A, 520, A54

Bessell, M. S. 1990, A\&AS, 83, 357

Bessell, M. S. 1991, AJ, 101, 662

Beuzit, J.-L., Ségransan, D., Forveille, T., et al. 2004, A\&A, 425, 997

Biller, B. A., \& Close, L. M. 2007, ApJL, 669, L41

Caballero, J. A. 2009, A\&A, 507, 251

Carson, J., Thalmann, C., Janson, M., et al. 2013, ApJL, 763, L32

Casagrande, L., Flynn, C., \& Bessell, M. 2008, MNRAS, 389, 585

Castro, S., Porto de Mello, G. F., \& da Silva, L. 1999, MNRAS, 305, 693

Chauvin, G., Lagrange, A.-M., Dumas, C., et al. 2004, A\&A, 425, L29

Cruz, K. L., Reid, I. N., Liebert, J., Kirkpatrick, J. D., \& Lowrance, P. J. 2003, AJ, 126, 2421

Cutri, R. M., Skrutskie, M. F., van Dyk, S., et al. 2003, 2MASS All Sky Catalog of Point Sources (NASA/IPAC Infrared Science Archive)

da Silva, L., Torres, C. A. O., de La Reza, R., et al. 2009, A\&A, 508, 833

Daemgen, S., Siegler, N., Reid, I. N., \& Close, L. M. 2007, ApJ, 654, 558

de la Reza, R., Torres, C. A. O., Quast, G., Castilho, B. V., \& Vieira, G. L. 1989, ApJL, 343, L61

Delfosse, X., Forveille, T., Beuzit, J., et al. 1999, A\&A, 344, 897

Delorme, P., Gagné, J., Girard, J. H., et al. 2013, A\&A, 553, L5

Delorme, P., Gagné, J., Malo, L., et al. 2012, A\&A, 548, A26

Desidera, S., Covino, E., Messina, S., et al. 2011, A\&A, 529, A54

Donati, J.-F., Catala, C., Landstreet, J. D., \& Petit, P. 2006, in ASP Conf. Ser. 358, Solar Polarization 4, ed. R. Casini \& B. W. Lites (San Francisco, CA: ASP), 362
Dotter, A., Chaboyer, B., Jevremović, D., et al. 2008, ApJS, 178, 89 Ducourant, C., Teixeira, R., Chauvin, G., et al. 2008, A\&A, 477, L1 Eggen, O. J. 1991, AJ, 102, 2028

Faherty, J. K., Burgasser, A. J., Walter, F. M., et al. 2012, ApJ, 752, 56

Famaey, B., Siebert, A., \& Jorissen, A. 2008, A\&A, 483, 453

Fischer, D. A., \& Marcy, G. W. 1992, ApJ, 396, 178

Franz, O. G., Henry, T. J., Wasserman, L. H., et al. 1998, AJ, 116, 1432

Gizis, J. E., \& Bharat, R. 2004, ApJL, 608, L113

Gizis, J. E., Jao, W., Subasavage, J. P., \& Henry, T. J. 2007, ApJL, 669, L45

Gizis, J. E., Reid, I. N., \& Hawley, S. L. 2002, AJ, 123, 3356

Graham, J. A. 1982, PASP, 94, 244

Gregorio-Hetem, J., Lepine, J. R. D., Quast, G. R., Torres, C. A. O., \& de La Reza, R. 1992, AJ, 103, 549

Hambly, N. C., Henry, T. J., Subasavage, J. P., Brown, M. A., \& Jao, W. 2004, AJ, 128,437

Henry, T. J., Ianna, P. A., Kirkpatrick, J. D., \& Jahreiss, H. 1997, AJ, 114, 388 Henry, T. J., Jao, W., Subasavage, J. P., et al. 2006, AJ, 132, 2360

Henry, T. J., Subasavage, J. P., Brown, M. A., et al. 2004, AJ, 128, 2460

Henry, T. J., Walkowicz, L. M., Barto, T. C., \& Golimowski, D. A. 2002, AJ, 123,2002

Hillenbrand, L. A., \& White, R. J. 2004, ApJ, 604, 741

Hinkle, K. H., Wallace, L., \& Livingston, W. 2003, BAAS, 35, 1260

Honeycutt, R. K. 1992, PASP, 104, 435

Jao, W., Henry, T. J., Subasavage, J. P., et al. 2005, AJ, 129, 1954

Jao, W., Henry, T. J., Subasavage, J. P., et al. 2011, AJ, 141, 117

Jao, W.-C., Henry, T. J., Beaulieu, T. D., \& Subasavage, J. P. 2008, AJ, 136, 840

Jao, W.-C., Henry, T. J., Subasavage, J. P., et al. 2003, AJ, 125, 332

Johnson, D. R. H., \& Soderblom, D. R. 1987, AJ, 93, 864

Kaufer, A., Stahl, O., Tubbesing, S., et al. 1999, Msngr, 95, 8

King, J. R., Villarreal, A. R., Soderblom, D. R., Gulliver, A. F., \& Adelman, S. J. 2003, AJ, 125, 1980

Kiss, L. L., Moór, A., Szalai, T., et al. 2011, MNRAS, 411, 117

Koen, C., Kilkenny, D., van Wyk, F., Cooper, D., \& Marang, F. 2002, MNRAS, 334,20

Landolt, A. U. 1992, AJ, 104, 340

Landolt, A. U. 2007, AJ, 133, 2502

Lépine, S., \& Simon, M. 2009, AJ, 137, 3632

López-Santiago, J., Micela, G., \& Montes, D. 2009, A\&A, 499, 129

Luhman, K. L., Stauffer, J. R., \& Mamajek, E. E. 2005, ApJL, 628, L69

Lyo, A.-R., Lawson, W. A., \& Bessell, M. S. 2004a, MNRAS, 355, 363

Lyo, A.-R., Lawson, W. A., \& Bessell, M. S. 2004b, MNRAS, 355, 363

Makarov, V. V., \& Urban, S. 2000, MNRAS, 317, 289

Malo, L., Doyon, R., Lafrenière, D., et al. 2013, ApJ, 762, 88

Mamajek, E. E. 2005, ApJ, 634, 1385

Mamajek, E. E., Bartlett, J. L., Seifahrt, A., et al. 2013, AJ, 146, 154

Marois, C., Macintosh, B., Barman, T., et al. 2008, Sci, 322, 1348

Mathieu, R. D., Baraffe, I., Simon, M., Stassun, K. G., \& White, R. 2007, in Protostars and Planets V, ed. B. Reipurth, D. Jewitt, \& K. Keil (Tucson, AZ: Univ. Arizona Press), 411

McArthur, B. E., Benedict, G. F., Harrison, T. E., \& van Altena, W. 2011, AJ, 141,172

McCarthy, K., \& White, R. J. 2012, AJ, 143, 134

Montes, D., López-Santiago, J., Fernández-Figueroa, M. J., \& Gálvez, M. C. 2001, A\&A, 379, 976

Nelan, E. P., Walborn, N. R., Wallace, D. J., et al. 2004, AJ, 128, 323

Perryman, M. A. C., Lindegren, L., Kovalevsky, J., et al. 1997, A\&A, 323, L49 Reid, I. N., \& Hawley, S. L. 1999, AJ, 117, 343

Reid, I. N., Hawley, S. L., \& Gizis, J. E. 1995, AJ, 110, 1838

Reiners, A., Basri, G., \& Browning, M. 2009, ApJ, 692, 538

Riaz, B., Gizis, J. E., \& Harvin, J. 2006, AJ, 132, 866

Rice, E. L., Faherty, J. K., \& Cruz, K. L. 2010, ApJL, 715, L165

Riedel, A. R. 2012, PhD thesis, Georgia State Univ.

Riedel, A. R., Murphy, S. J., Henry, T. J., et al. 2011, AJ, 142, 104

Riedel, A. R., Subasavage, J. P., Finch, C. T., et al. 2010, AJ, 140, 897

Rodriguez, D. R., Bessell, M. S., Zuckerman, B., \& Kastner, J. H. 2011, ApJ, 727, 62

Rojas-Ayala, B., Covey, K. R., Muirhead, P. S., \& Lloyd, J. P. 2010, ApJL, 720, L113

Röser, S., Schilbach, E., Piskunov, A. E., Kharchenko, N. V., \& Scholz, R.-D. 2011, A\&A, 531, A92

Schlieder, J. E. 2011, PhD thesis, State University of New York at Stony Brook Schlieder, J. E., Lépine, S., Rice, E., et al. 2012a, AJ, 143, 114

Schlieder, J. E., Lépine, S., \& Simon, M. 2010, AJ, 140, 119

Schlieder, J. E., Lépine, S., \& Simon, M. 2012b, AJ, 143, 80

Schlieder, J. E., Lépine, S., \& Simon, M. 2012c, AJ, 144, 109

Schmitt, J. H. M. M., Fleming, T. A., \& Giampapa, M. S. 1995, ApJ, 450, 392

Scholz, R., Lo Curto, G., Méndez, R. A., et al. 2005, A\&A, 439, 1127 
Shkolnik, E., Liu, M. C., \& Reid, I. N. 2009, ApJ, 699, 649

Shkolnik, E. L., Anglada-Escudé, G., Liu, M. C., et al. 2012, ApJ, 758, 56

Shkolnik, E. L., Hebb, L., Liu, M. C., Reid, I. N., \& Collier Cameron, A. 2010, ApJ, 716, 1522

Shkolnik, E. L., Liu, M. C., Reid, I. N., Dupuy, T., \& Weinberger, A. J. 2011, ApJ, 727, 6

Skrutskie, M. F., Cutri, R. M., Stiening, R., et al. 2006, AJ, 131, 1163

Soderblom, D. R., Nelan, E., Benedict, G. F., et al. 2005, AJ, 129, 1616

Song, I., Zuckerman, B., \& Bessell, M. S. 2003, ApJ, 599, 342

Torres, C. A. O., Quast, G. R., da Silva, L., et al. 2006, A\&A, 460,695

Torres, C. A. O., Quast, G. R., de La Reza, R., et al. 2003, in Open Issues in Local Star Formation, ed. J. Lépine \& J. Gregorio-Hetem (Astrophysics and Space Science Library, Vol. 299; Dordecht: Kluwer), 83

Torres, C. A. O., Quast, G. R., Melo, C. H. F., \& Sterzik, M. F. 2008, Handbook of Star Forming Regions, Volume II (San Francisco, CA: ASP), 757

van Altena, W. F., Lee, J. T., \& Hoffleit, E. D. 1995, The General Catalogue of Trigonometric [Stellar] Parallaxes, Completely Revised and Enlarged (4th ed.; New Haven, CT: Yale Univ. Observatory) van Leeuwen, F. (ed.) 2007, Hipparcos, the New Reduction of the Raw Data (Astrophysics and Space Science Library, Vol. 350; Berlin: Springer)

Voges, W., Aschenbach, B., Boller, T., et al. 1999, A\&A, 349, 389

Voges, W., Aschenbach, B., Boller, T., et al. 2000, IAU Circ., 7432, 1

Webb, R. A., Zuckerman, B., Platais, I., et al. 1999, ApJL, 512, L63

Weinberger, A. J., Anglada-Escudé, G., \& Boss, A. P. 2013, ApJ, 762, 118

Weis, E. W. 1993, AJ, 105, 1962

Weis, E. W. 1996, AJ, 112, 2300

Weis, E. W., Lee, J. T., Lee, A. H., et al. 1999, AJ, 117, 1037

West, A. A., \& Basri, G. 2009, ApJ, 693, 1283

West, A. A., Hawley, S. L., Bochanski, J. J., et al. 2008, AJ, 135, 785

White, R. J., \& Basri, G. 2003, ApJ, 582, 1109

Winters, J. G., Henry, T. J., Jao, W., et al. 2011, AJ, 141, 21

Yee, J. C., \& Jensen, E. L. N. 2010, ApJ, 711, 303

Zacharias, N., Finch, C. T., Girard, T. M., et al. 2013, AJ, 145, 44

Zuckerman, B., Rhee, J. H., Song, I., \& Bessell, M. S. 2011, ApJ, 732, 61

Zuckerman, B., \& Song, I. 2004, ARA\&A, 42, 685

Zuckerman, B., Song, I., \& Bessell, M. S. 2004, ApJL, 613, L65

Zuckerman, B., Song, I., \& Webb, R. A. 2001, ApJ, 559, 388 